\title{
Turbo Equalization: An Overview
}

\author{
Michael Tüchler and Andrew C. Singer, Fellow, IEEE
}

Dedicated to the memory of Ralf Koetter (1963-2009)

\begin{abstract}
Turbo codes and the iterative algorithm for decoding them sparked a new era in the theory and practice of error control codes. Turbo equalization followed as a natural extension to this development, as an iterative technique for detection and decoding of data that has been both protected with forward error correction and transmitted over a channel with intersymbol interference (ISI). In this paper, we review the turbo equalization approach to coded data transmission over ISI channels, with an emphasis on the basic ideas, some of the practical details, and many of the research directions that have arisen from this offshoot, introduced by Douillard, et al. of the original turbo decoding algorithm. The subsequent relaxation of the maximum a posteriori (MAP) equalization algorithm to include linear and other simpler receivers sparked a decade and a half of research into iterative algorithms, spanning research problems ranging from trellis coded modulation to underwater acoustic communications.
\end{abstract}

Index Terms-Equalization, iterative decoding, minimum mean square error, turbo equalization.

\section{Turbo Equalization OvervieW}

I $\mathrm{N}$ this paper, we discuss the turbo equalization approach to coded data transmission over intersymbol interference (ISI) channels. Our emphasis is on the basic ideas and some of the practical details involved in making use of this offshoot of turbo-decoding that has sparked a number of interesting new research directions in the fifteen years since its discovery.

We begin with a high-level overview of turbo equalization and bring our attention to a digital communication system, whose transmitter is depicted in Fig. 1. The components in this system are typical to most practical digital communication links and are essential to the application of turbo equalization in the receiver.

The receiver for this system must detect the data that was transmitted, making use of knowledge of the channel together with the available redundancy introduced to protect the data, in

Manuscript received April 25, 2010; revised October 30, 2010; accepted November 11, 2010. Date of current version January 19, 2011. This work was supported in part by the Department of the Navy, Office of Naval Research, under Grant ONR MURI N00014-07-1-0738 and Grant ONR N00014-07-1-0311, in part by the National Science Foundation under Grant NSF CCF 07-29092, and in part by the Gigascale System Research Center (GSRC), one of five research centers funded under the Focus Center Research Program (FCRP), a Semiconductor Research Corporation program. This paper contains material from the doctoral dissertation of M. Tüchler, "Turbo equalization,” Munich Univ. of Technol, Munich, Germany, Dec. 2003.

This paper is part of the special issue on "Facets of Coding Theory: From Algorithms to Networks," dedicated to the scientific legacy of Ralf Koetter.

M. Tüchler is with Rheinmetall AG, 8050 Zurich, Switzerland.

A. C. Singer is with the Department of Electrical and Computer Engineering, University of Illinois at Urbana-Champaign, Urbana IL 61801 USA.

Communicated by F. R. Kschischang, Associate Editor for the special issue on "Facets of Coding Theory: From Algorithms to Networks."

Digital Object Identifier 10.1109/TIT.2010.2096033

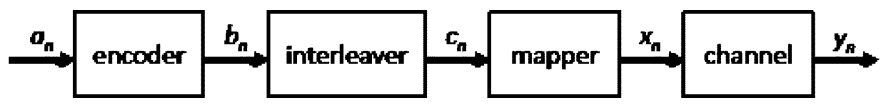

Fig. 1. Transmitter configuration of a digital communication system.

the form of forward error correction (FEC). Mitigating the effects of an intersymbol interference (ISI) channel on the transmitted data is generally called equalization or detection, while the subsequent problem of recovering the data from the equalized symbols, making use of the FEC, is called decoding. For complexity reasons, these problems have typically been considered separately, with limited interaction between the two blocks. As such, substantial performance degradation can be induced. The main contribution of much of the work in turbo equalization to date has been to enable feasible approaches to jointly addressing the equalization and decoding tasks. As a result, the performance gap between an optimal joint equalization and decoding and that achievable through systems with practical complexity has been narrowed in a manner similar to that of near Shannon-limit communications using turbo codes [1]. Such performance gains can be anticipated for any communication link in which joint equalization and decoding would outperform separate detection and decoding, such as would be the case for intersymbol interference channels employing a single-carrier modulation.

This paper is organized as follows: Section I begins with an overview of turbo equalization and shows some of the many applications and extensions to the original formulation of [2] that have arisen over the last decade and a half. For ease of description, Section II provides an overview of turbo equalization as applied to systems using BPSK modulation, and Section III applies these developments to more general QAM alphabets. Implementation issues including low-complexity approximations of the receiver structures are described in Section IV. The performance enhancing benefits of precoding are discussed in Section V.

An equalizer produces estimates of the transmitted symbols and, for complexity reasons, often consists of linear processing of the received signal and possibly past symbol estimates. The parameters of these equalizers can be optimized using a variety of optimization algorithms, such as zero forcing (ZF) or minimum mean squared error (MMSE) estimation [3], [4]. Methods for minimizing the bit error rate (BER) or the sequence error rate (SER) are highly nonlinear, and are based on maximum likelihood (ML) estimation, which turns into maximum a posteriori probability (MAP) estimation in presence of a priori information about the transmitted data. Efficient algorithms exist for MAP/ML sequence estimation, such as the Viterbi algorithm [3], [5], [6], for MAP/ML symbol estimation, such as the 
BCJR algorithm [7]. However, the complexity of such methods often remains significantly higher than that for linear methods. This is true in particular for channels with large delay spread (memory) $M_{h}$ or given a large size $|\mathcal{S}|$ of the signal alphabet, since the receiver complexity is of order $O\left(|\mathcal{S}|^{M_{h}}\right)$ per symbol. State-of-the-art systems for a variety of communication channels employ convolutional codes and ML equalizers together with an interleaver after the encoder and a deinterleaver before the decoder [8], [9]. Interleaving shuffles symbols within a given block of data to decorrelate error events introduced, or unresolved, by the equalizer between neighboring symbols. These error bursts are hard to mitigate using, for example, a convolutional decoder alone.

A number of iterative receiver algorithms repeat the equalization and decoding tasks on the same set of received data, where feedback information from the decoder is incorporated into the equalization process. This method is the basis of turbo equalization and is based on decoding methods for turbo codes [10]-[12] and has close connections to the iterative algorithms used in conjunction with so-called factor graphs [13]-[16]. This approach has been adapted to various communication tasks, ranging from detection and decoding of trellis coded modulation (TCM) [17], [18] and code division multiple access (CDMA) [19] to underwater acoustic communications [20]-[23] and optical fiber communications [24]-[26]. Over the last decade and a half, this joint, iterative approach has been applied to a vast array of problems that involve the use of an error control code to aid in the processing of another related task in the receive chain of a digital communication link. Turbo equalization systems were first proposed in [2], by noting that the transmitter in Fig. 1 can be viewed as employing a serially concatenated convolutional code (turbo code), where the inner code is taken over the reals (by means of the ISI channel), and further developed in a number of articles, including [27]-[30]. In some of these systems, MAP-based techniques, as well as a Viterbi algorithm producing soft output information [31], were used for both equalization and decoding [2], [27]. The more complex BCJR algorithm [7] was implemented in [27]. These tasks are similar enough in structure, that in [32], an architecture was developed such that the same circuitry could be used for both, after which other high-throughput circuit implementations have also appeared [33]-[38].

The approaches in [29], [30], [39]-[41] address a major shortcoming of the classical turbo equalization scheme [2], [27], [28], which is the exponentially increasing complexity of the equalizer for channels with long memory or given a large signal alphabet. These replace the MAP equalizer with a linear or a decision feedback equalizer. The approach in [39] and later [42], replaces the MAP equalizer in the turbo equalization framework by a soft interference canceler, obtained using a leastmean-square (LMS) based update algorithm. An added benefit of this approach is its ability to track changes in the channel over time. However, this comes at the expense of using a single linear filter that does not adjust to the soft-feedback from the decoder, a property that the MMSE-based linear methods possess [29], [30]. In [43], an extending window version of the slidingwindow MMSE approach in [29], [30] exploits the added structure to further reduce complexity.
Another common technique to reduce the complexity of the MAP equalizer is to reduce the number of states in the underlying trellis, which was applied to turbo equalization in [44]. In [45], MAP complexity is reduced by exploring only the most promising paths in the trellis, exploiting properties of the channel in this selection. In [46], [47], the MAP complexity is reduced by replacing the BCJR algorithm with an approximation based on Markov chain Monte Carlo methods, and in [48], the BCJR algorithm is replaced by an approach based on simulated annealing. A local search over a suitably defined objective function is used to approximate MAP detection in [49].

Reduced complexity turbo equalization methods have been applied to magnetic recording channels, where in [50] a soft decision feedback equalizer is used and in [51] 2-D MMSE turbo equalization is used along with LDPC codes. The special case of 2-D separable ISI was considered in [52] and in [53] overlapping tracks are considered.

The original work of [19] pioneered a turbo equalization-like approach to multiuser detection in CDMA using linear receivers in place of the MAP detector. A number of extensions of this work followed, including a reduced-state trellis search approach to mitigating ISI and multiple access interference (MAI) in [54]. For mitigating intercell and intersymbol interference, a distributed turbo equalization approach was explored in [55]. For multiple-access links that include ISI, MAI, and multiple antennas, a variational inference approach was used to approximate MAP detection in [56]. When such MIMO channels are rapidly fading, improved convergence speed was achieved through the use of linear dispersion codes in [57].

Based on the multiuser formulation of the problem, a natural extension to multiple-input/multiple-output (MIMO) channels arose and a host of results were also developed in this area, including methods for suppressing multiuser interference [58]-[60], accounting for channel estimation errors [61], and results from a number of so-called turbo-BLAST experimental systems followed, e.g., [62]. Extensions to trellis-coded modulation [63] and BICM [64] as well as frequency domain-based formulations using single-carrier transmission with and without cyclic prefix [65]-[69] as well as MIMO OFDM were also developed [70], [71]. The increase in problem dimensionality lends itself naturally to complexity reduction methods through rank reduction [72] and a variety of other MAP approximation methods.

One application area in which turbo equalziation methods have provided substantial gains over systematically separate equalization and decoding is that of underwater acoustic communications [21], [23]. The long delay-spread of the channel (several tens to hundreds of symbol periods) makes MAP-based methods prohibitive and poses real challenges for MMSE-based linear methods as well. In addition, the underwater acoustic environment is often rapidly time-varying, such that explicit channel estimation and tracking must be employed [73]. A variety of methods for incorporating soft information for iteratively estimating the channel have been developed, such as those based on a Kalman formulation [74], [75]. The relatively long delay-spread of the channel makes OFDM-based methods appear attractive, however this gives rise to intercarrier 


\section{Section Guide}

\begin{tabular}{|c|c|c|c|c|}
\hline & \multicolumn{2}{|c|}{ BPSK } & \multicolumn{2}{c|}{ PSK / QAM } \\
\hline Type & Sections & Tables $O$ (complexity) & Sections & Tables $O($ complexity) \\
\hline Trellis-based & II.D, IV.A & I $O\left(L \cdot 2^{M_{h}}\right)$ & III.B & $O\left(L \cdot|S|^{M_{h}}\right)$ \\
\hline MMSE & II.E, IV.B & III $O\left(L \cdot W^{3}\right)$, IV $O\left(L \cdot W^{2}\right)$ & III.C & V $O\left(L \cdot W^{3}\right)$, VIII $O\left(L \cdot W^{2}\right)$ \\
\hline Approximate & IV.C, IV.D & VI $O(L \cdot W)$, VII $O(L \cdot \ln L)$ & IV.F & IX $O(L \cdot W)$, X $O(L \cdot \ln L)$ \\
\hline
\end{tabular}

interference (ICI) due to the time variation and, once again, a linear channel matrix arises, for which ISI is replaced by ICI. A variety of joint ICI-mitigation/decoding methods have been developed for performing turbo equalization over such time-varying channels using OFDM [76]-[78].

In the sequel, we consider the turbo equalization approach as applied to simple BPSK modulation as well as higher order alphabets. Additionally, we consider several approaches to realizing the turbo equalization algorithm, ranging from trellis-based algorithms that make use of the BCJR algorithm to approaches based on MMSE linear equalization as well as low complexity implementations and approximations based on time-domain and frequency domain formulations. As a guide to the reader, the Section Guide above provides an overview of where each of these topics is discussed, together with a listing of tables containing pseudocode algorithmic descriptions for each case considered and an approximate complexity, per equalization iteration, for a block of length $L$ symbols from an alphabet of size $|S|$ for a channel of memory $M_{h}$, assuming an equalizer with support $W$.

\section{COMmUnication Systems USING BPSK MOdULATION}

\section{A. System Model}

This section defines a specific configuration of the transmitter of Fig. 1. In particular, the real-valued BPSK signal alphabet $\mathcal{S}=\{+1,-1\}$ and an ISI channel with real-valued impulse response $h[n]$ is considered. Based on these results, algorithms for arbitrary complex-valued signal alphabets $\mathcal{S}$ and channel impulse responses $h[n]$ are given in Section III.

A data source produces independent and uniformly distributed (IUD) data bits $a_{n}$. The sequence $\mathbf{a}=\left(a_{0} a_{1} \ldots a_{K-1}\right)$ of $K$ data bits is protected by a memory $M_{c}=2$ convolutional FEC code defined by the generator polynomials $g_{0}(D)=1+D^{2}$ and $g_{1}(D)=1+D+D^{2}$. The encoding operation yielding the $N$ code bits $\mathbf{b}=\left(b_{0} b_{1} \ldots b_{N-1}\right)$ is carried out systematically including trellis termination. It follows that the FEC code rate is equal to

$$
R_{\mathrm{fec}}=\frac{K}{N}=\frac{K}{2(K+2)}=\frac{1}{2+2 / K} .
$$

Fig. 2 depicts a trellis for this encoder with the branch labeling $a_{n} / b_{2 n} b_{2 n+1}$. A corresponding state-space model utilizing the two-dimensional state variable $\theta_{n}$ is given by

$$
\begin{aligned}
\theta_{n+1} & =\left[\begin{array}{ll}
0 & 1 \\
1 & 0
\end{array}\right] \theta_{n}+\left[\begin{array}{l}
1 \\
0
\end{array}\right] a_{n}, \\
{\left[\begin{array}{c}
b_{2 n} \\
b_{2 n+1}
\end{array}\right] } & =\left[\begin{array}{ll}
0 & 0 \\
1 & 1
\end{array}\right] \theta_{n}+\left[\begin{array}{l}
1 \\
1
\end{array}\right] a_{n}, \quad n=0,1, \ldots, K+1 .
\end{aligned}
$$

The initial state $\theta_{0}$ is $\mathbf{0}_{2}$ and $a_{K}=a_{K+1}=0$ (termination bits). The code bits $b_{n}$ are permuted using an S-random interleaver [11] to the $N$ bits $\mathbf{c}=\left(c_{0} c_{1} \ldots c_{N-1}\right)$, which are modulated to the symbols $\mathbf{x}=\left(x_{0} x_{1} \ldots x_{L-1}\right)^{\mathrm{T}}$ using BPSK modulation. It follows that the transmitted symbols $x_{n}$ are given by

$$
x_{n}= \begin{cases}+1, & c_{n}=0 \\ -1, & c_{n}=1\end{cases}
$$

The system code rate $R$ is given by

$$
R=\log _{2}|\mathcal{S}| \cdot R_{\mathrm{fec}}=R_{\mathrm{fec}}=\frac{1}{2+2 / K} .
$$

The additive white Gaussian noise (AWGN) ISI channel model is given by

$$
\begin{gathered}
y_{n}=w_{n}+\sum_{i=0}^{M_{h}} h_{i} x_{n-i}, \\
n=0,1, \ldots, L-1, \quad \text { or } \quad \mathbf{y}=\mathbf{H x}+\mathbf{w},
\end{gathered}
$$

where $\mathbf{y}=\left(y_{0} y_{1} \ldots y_{L-1}\right)^{\mathrm{T}}$ and $\mathbf{w}=\left(w_{0} w_{1} \ldots w_{L-1}\right)^{\mathrm{T}}$ for $L=N$. The channel coefficients $h_{i}$ and the noise samples $w_{n}$ are assumed to be real-valued, i.e., the $\operatorname{PDF} p\left(w_{n}\right)$ is given by $\mathcal{N}_{\mathbb{R}}\left(0, \sigma^{2}\right)$, where $\mathcal{N}_{\mathbb{R}}\left(\mu, \sigma^{2}\right)$ denotes

$$
p(x)=\exp \left(-(x-\mu)^{2} /\left(2 \sigma^{2}\right)\right) / \sqrt{2 \pi \sigma^{2}} .
$$

The $L \times L$ system matrix $\mathbf{H}$ has different structures depending on how the undefined symbols $x_{-1}, x_{-2}, \ldots, x_{-M_{h}}$ in (1) are treated. For example, they are 0 under the termination assumption or they are given by $x_{L-1}, x_{L-2}, \ldots, x_{L-M_{h}}$ under the periodic extension assumption. 

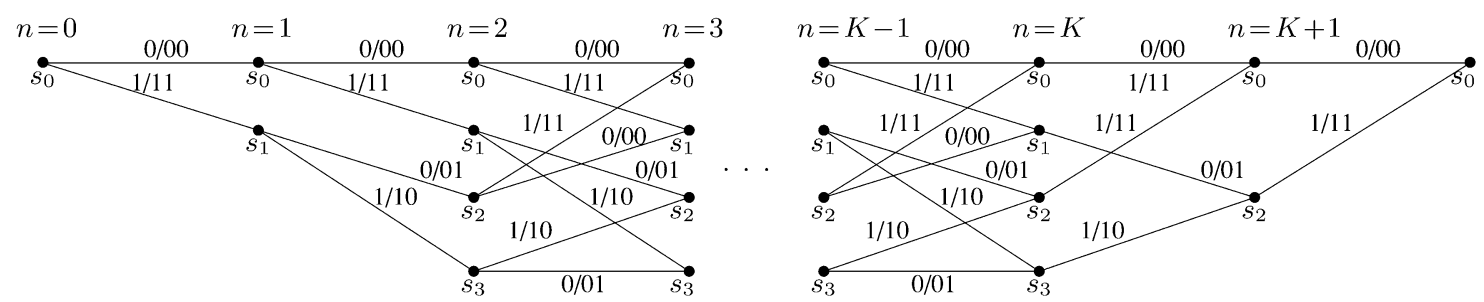

Fig. 2. Trellis for systematic encoding of a convolutional code given by the generator polynomials $g_{0}(D)=1+D^{2}$ and $g_{1}(D)=1+D+D^{2}$.
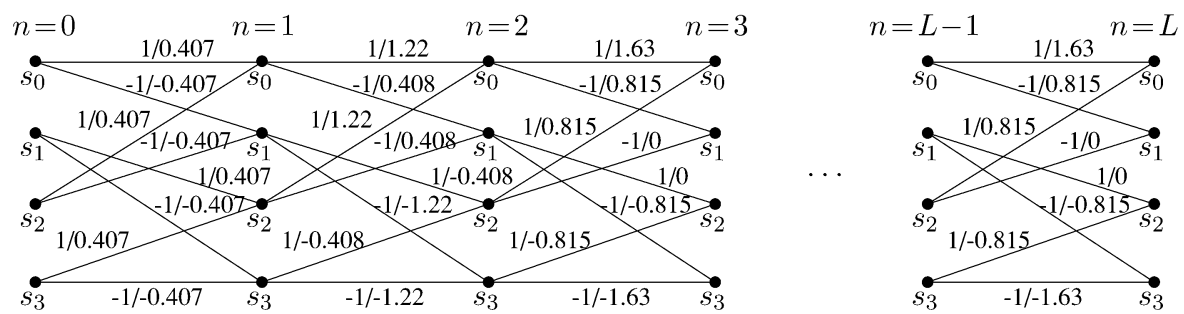

Fig. 3. Trellis for a length-3 ISI channel given by the coefficients $h_{0}=0.407, h_{1}=0.815$, and $h_{2}=0.407$ under the termination assumption.

To illustrate performance, the length- 3 unit power channel defined by the coefficients $h_{0}=0.407, h_{1}=0.815$, and $h_{2}=$ 0.407 is used as an example channel. This channel has memory $M_{h}=2$. Its system matrix $\mathbf{H}$ is given by

$$
\begin{aligned}
& \text { termination assumption: } \\
& \mathbf{H}=\left[\begin{array}{ccccccc}
h_{0} & 0 & 0 & 0 & 0 & \cdots & 0 \\
h_{1} & h_{0} & 0 & 0 & 0 & \cdots & 0 \\
h_{2} & h_{1} & h_{0} & 0 & 0 & \cdots & 0 \\
& & \ddots & \ddots & \ddots & & \\
0 & 0 & \cdots & 0 & h_{2} & h_{1} & h_{0}
\end{array}\right],
\end{aligned}
$$

periodic extension assumption:

$$
\mathbf{H}=\left[\begin{array}{cccccccc}
h_{0} & 0 & 0 & 0 & 0 & \cdots & h_{2} & h_{1} \\
h_{1} & h_{0} & 0 & 0 & 0 & \cdots & 0 & h_{2} \\
h_{2} & h_{1} & h_{0} & 0 & 0 & \cdots & 0 & 0 \\
& & \ddots & \ddots & \ddots & & & \\
0 & 0 & \cdots & 0 & 0 & h_{2} & h_{1} & h_{0}
\end{array}\right] .
$$

Unless otherwise specified in the sequel, the termination assumption will be applied. The periodic extension assumption is applied in Section IV to derive low-complexity equalization algorithms based on MMSE estimation in the frequency domain. Fig. 3 depicts a trellis with the branch labeling $x_{n} / v_{n}$ describing the length-3 example channel.

\section{B. Optimal Detection}

The BEP-optimal decoder computes estimates $\hat{a}_{n}$ of the data bits $a_{n}$ minimizing bit error probability (BEP) $\operatorname{Pr}\left\{a_{n} \neq \hat{a}_{n}\right\}$

$$
\hat{a}_{n}=\underset{a \in \mathbb{F}_{2}}{\operatorname{argmax}} P\left(a_{n}=a \mid \mathbf{y}\right), \quad \text { for } a=0,1, \ldots, K-1 .
$$

The posterior probabilities $P\left(a_{n} \mid \mathbf{y}\right)$ follow from marginalizing over $a_{n}$ in the sequence-based posterior probability $P(\mathbf{a} \mid \mathbf{y})$

$$
P\left(a_{n}=a \mid \mathbf{y}\right)=\sum_{\mathbf{a} \in \mathbb{F}_{2}^{K !} ! a_{n}=a} P(\mathbf{a} \mid \mathbf{y})
$$

$$
=\frac{1}{p(\mathbf{y})} \cdot \sum_{\mathbf{a} \in \mathbb{F}_{2}^{K}: a_{n}=a} p(\mathbf{y} \mid \mathbf{a}) \cdot P(\mathbf{a}) .
$$

The IUD assumption on the bits $a_{n}$ yields that $P(\mathbf{a})$ factors into $\prod_{n=0}^{K-1} P\left(a_{n}\right)$.

When binary random variables are concerned, it is convenient to work with log-likelihood ratios (LLRs) rather than probabilities [79]. Consider the conditional LLR $L\left(a_{n} \mid \mathbf{y}\right)$ of $a_{n}$ given $\mathbf{y}$

$$
L\left(a_{n} \mid \mathbf{y}\right)=\ln \frac{P\left(a_{n}=0 \mid \mathbf{y}\right)}{P\left(a_{n}=1 \mid \mathbf{y}\right)} .
$$

The decoding rule (3) can be rewritten to

$$
\hat{a}_{n}=\left\{\begin{array}{ll}
0, & L\left(a_{n} \mid \mathbf{y}\right) \geq 0 \\
1, & L\left(a_{n} \mid \mathbf{y}\right)<0
\end{array} \quad \text { for } \quad a=0,1, \ldots, K-1 .\right.
$$

From (4) it follows that $L\left(a_{n} \mid \mathbf{y}\right)$ can be decomposed into an extrinsic and an a priori LLR

$$
\begin{aligned}
L\left(a_{n} \mid \mathbf{y}\right) & \\
= & \ln \frac{\sum_{\mathbf{a}: a_{n}=0} p(\mathbf{y} \mid \mathbf{a}) \prod_{k=0}^{K-1} P\left(a_{k}\right)}{\sum_{\mathbf{a}: a_{n}=1} p(\mathbf{y} \mid \mathbf{a}) \prod_{k=0}^{K-1} P\left(a_{k}\right)} \\
= & \underbrace{\ln \frac{\sum_{\mathbf{a}: a_{n}=0} p(\mathbf{y} \mid \mathbf{a}) \prod_{k=0: k \neq n}^{K-1} P\left(a_{k}\right)}{\sum_{\mathbf{a}: a_{n}=1} p(\mathbf{y} \mid \mathbf{a}) \prod_{k=0: k \neq n}^{K-1} P\left(a_{k}\right)}} \\
& +\underbrace{\ln \frac{P\left(a_{n}=0\right)}{P\left(a_{n}=1\right)}} \\
= & L_{\text {ext }}\left(a_{n} \mid \mathbf{y}\right)+L\left(a_{n}\right) .
\end{aligned}
$$

The LLR $L_{\text {ext }}\left(a_{n} \mid \mathbf{y}\right)$ represents information about $a_{n}$ contained in $\mathbf{y}$ and $P\left(a_{k}\right)$ for all $k \neq n$, which is the so-called extrinsic information or the extrinsic LLR, respectively. It is added to the a priori LLR $L\left(a_{n}\right)$, which represents the available $a$ priori information about $a_{n}$. Extrinsic LLRs play a crucial role in turbo equalization. Unfortunately the BEP-optimal decoder (6) has computational complexity that is intractible for large $K$ and is of order $O\left(2^{K}\right)$. 

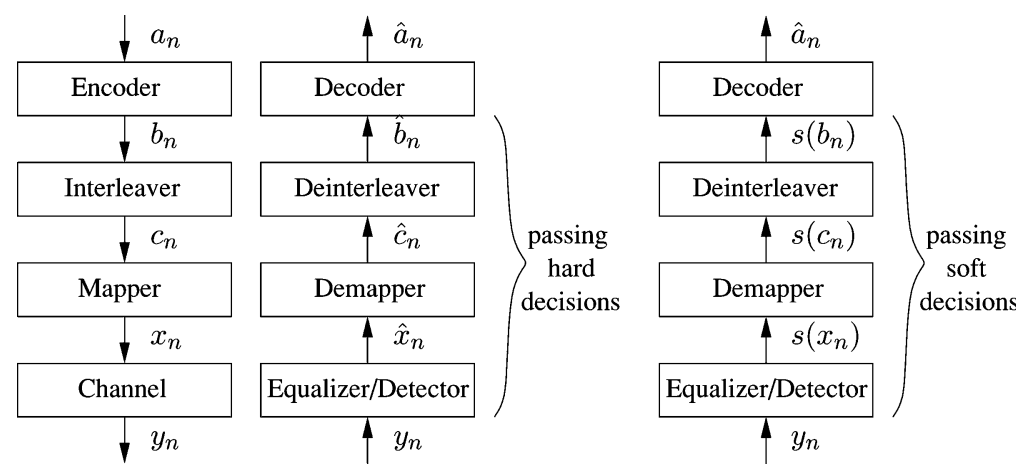

Fig. 4. Receiver structure for separate equalization and decoding.

\section{Separate Equalization and Decoding}

A standard approach to reducing the computational burden of the receiver is to split the detection problem into the two subproblems equalization (detection) and decoding. This strategy is employed in the two receiver structures depicted in Fig. 4. The difference between them is that the receiver on the left communicates estimates $\hat{x}_{n}, \hat{c}_{n}$, and $\hat{b}_{n}$ from the same alphabet as $x_{n}, c_{n}$, and $b_{n}$, respectively, from the equalizer to the decoder whereas the receiver on the right use so-called soft information $s\left(x_{n}\right), s\left(c_{n}\right)$, and $s\left(b_{n}\right)$.

Two common, but distinct families of algorithms for the subproblem of equalization are those based on trellis methods and those using linear filters. Typical trellis-based approaches are symbol-based or sequence-based MAP/ML detection. A symbol-based MAP detector computes estimates $\hat{x}_{n}$ of the symbols $x_{n}$ as follows:

$$
\hat{x}_{n}=\underset{x \in \mathcal{S}}{\operatorname{argmax}} P\left(x_{n}=x \mid \mathbf{y}\right) .
$$

This can be done efficiently using the BCJR algorithm [7] as long as the ISI channel has a trellis with a sufficiently small number of states. An overview of BCJR-based turbo equalization is given in [80]. Table I applies this description to the example ISI channel defined in Section II-A, where $1_{L}$ denotes a length- $L$ column vector containing all ones and $\odot$ denotes element-wise multiplication. The entries $\gamma_{n}\left(s_{i}, s_{j}\right)=0.5 \cdot p\left(y_{n} \mid v_{n}=v_{i, j}\right)$ of $\boldsymbol{\Gamma}_{n}$ and the initial vectors $\mathbf{f}_{0}$ and $\mathbf{b}_{L}$ follow from (1), and the ISI channel trellis in Fig. 3. The scaling of $\gamma_{n}\left(s_{i}, s_{j}\right)$ by $0.5 / \sqrt{2 \pi \sigma^{2}}$ has been neglected because of the normalization applied in the forward and backward recursion. Practical implementations of the BCJR algorithm often store the logarithms of the quantities in Table I [12], [81]. In this case, it is possible to use the terms $-\left|y_{n}-v_{i, j}\right|^{2} /\left(2 \sigma^{2}\right)$ directly in $\ln \boldsymbol{\Gamma}_{n}$ without the need for exponentiation. This can be accomplished exactly, for example, using the max-star operator as in [82], or approximately by making the approximation $\ln \left(\sum_{k} e^{a_{k}}\right) \approx \max _{k} a_{k}$.

A sequence-based MAP detector computes an estimate $\hat{\mathbf{x}}$ of the sequence $\mathbf{x}$ as follows:

$$
\hat{\mathbf{x}}=\underset{\mathbf{x} \in \mathcal{S}^{L}}{\operatorname{argmax}} P(\mathbf{x} \mid \mathbf{y}) .
$$

This can be done efficiently using the Viterbi algorithm [6], [83].

The computational complexity of the trellis-based approaches is determined by the number $|\mathcal{S}|^{M_{h}}$ of trellis states, which grows with the size $|\mathcal{S}|$ of the signal alphabet and exponentially with the memory $M_{h}$ of the ISI channel. Note that the trellis-based implementation of MAP detection (as opposed to exhaustive search) in Table I is possible only because the symbols $x_{n}$ are assumed to be independent, i.e., $P(\mathbf{x})$ factors into $\prod_{n=0}^{L-1} P\left(x_{n}\right)$. The interleaver between the encoder and the ISI channel provides justification for this assumption and enables separate MAP detection and MAP decoding using trellis-based algorithms to be used.

Linear filter-based approaches perform only simple operations on the received symbols, which can be described with matrix operations on the sequence $y$ directly. The matrix formulation (1) of the ISI channel model immediately suggests multiplying the received symbols $\mathbf{y}$ with an (approximate) inverse matrix $\mathbf{H}^{-1}$ yielding an estimate $\hat{\mathbf{x}}$ of the sequence $\mathbf{x}$. This so-called zero forcing (ZF) equalizer [83] attempts to invert the channel and may suffer from noise enhancement, which can be severe if $\mathbf{H}$ is ill-conditioned. Noise enhancement can be avoided using (linear) minimum mean square error (MMSE) estimation [84]. The estimate $\hat{\mathbf{x}}$ of $\mathbf{x}$ minimizing the MSE $\mathrm{E}\{\| \hat{\mathbf{x}}-$ $\left.\mathbf{x}\|\|^{2}\right\}$ is given by

$$
\hat{\mathbf{x}}=\mathrm{E}\{\mathbf{x}\}+\operatorname{Cov}\{\mathbf{x}, \mathbf{y}\} \operatorname{Cov}\{\mathbf{y}, \mathbf{y}\}^{-1}(\mathbf{y}-\mathrm{E}\{\mathbf{y}\}),
$$

where $\operatorname{Cov}\{\mathbf{x}, \mathbf{y}\}=\mathrm{E}\left\{\mathbf{x y}^{H}\right\}-\mathrm{E}\{\mathbf{x}\} \mathrm{E}\{\mathbf{y}\}^{H}$. Since each entry in the noise sequence $\mathbf{w}$ is IUD with $\mathcal{N}_{\mathbb{R}}\left(0, \sigma^{2}\right)$, it follows that $\operatorname{Cov}\{\mathbf{w}, \mathbf{w}\}$ equals $\sigma^{2} \mathbf{I}_{L}$. From the IUD assumption on the symbols $x_{n}$, it follows that the estimate $\hat{\mathrm{x}}$ is therefore given by

$$
\hat{\mathbf{x}}=\mathbf{H}^{\mathrm{T}}\left(\sigma^{2} \mathbf{I}_{L}+\mathbf{H H}^{\mathrm{T}}\right)^{-1} \mathbf{y} .
$$

Since the symbol estimates $\hat{x}_{n}$ in $\hat{\mathbf{x}}$ are most often not from the signal alphabet $\mathcal{S}$, they are usually mapped to that symbol from $\mathcal{S}$ at closest (Euclidean) distance to $\hat{x}_{n}$. Note that in a practical implementation of (8), only a small window of received symbols $y_{n}$ rather than the complete sequence $\mathbf{y}$ is considered for complexity reasons.

The equalizer can often provide more information to the decoder than the hard decisions $\hat{x}_{n}$ at the cost of additional storage, processing, and communication, such as probabilities that $x_{n}$ takes on a particular symbol from $\mathcal{S}$. The principle of using probabilities (soft information) rather than hard-decisions is often referred to as soft processing or soft decoding. This is 
TABLE I

Algorithm for Computing the Posterior Probabilities $P\left(x_{n} \mid \mathbf{y}\right)$ FOR the ISI Channel Model (1)

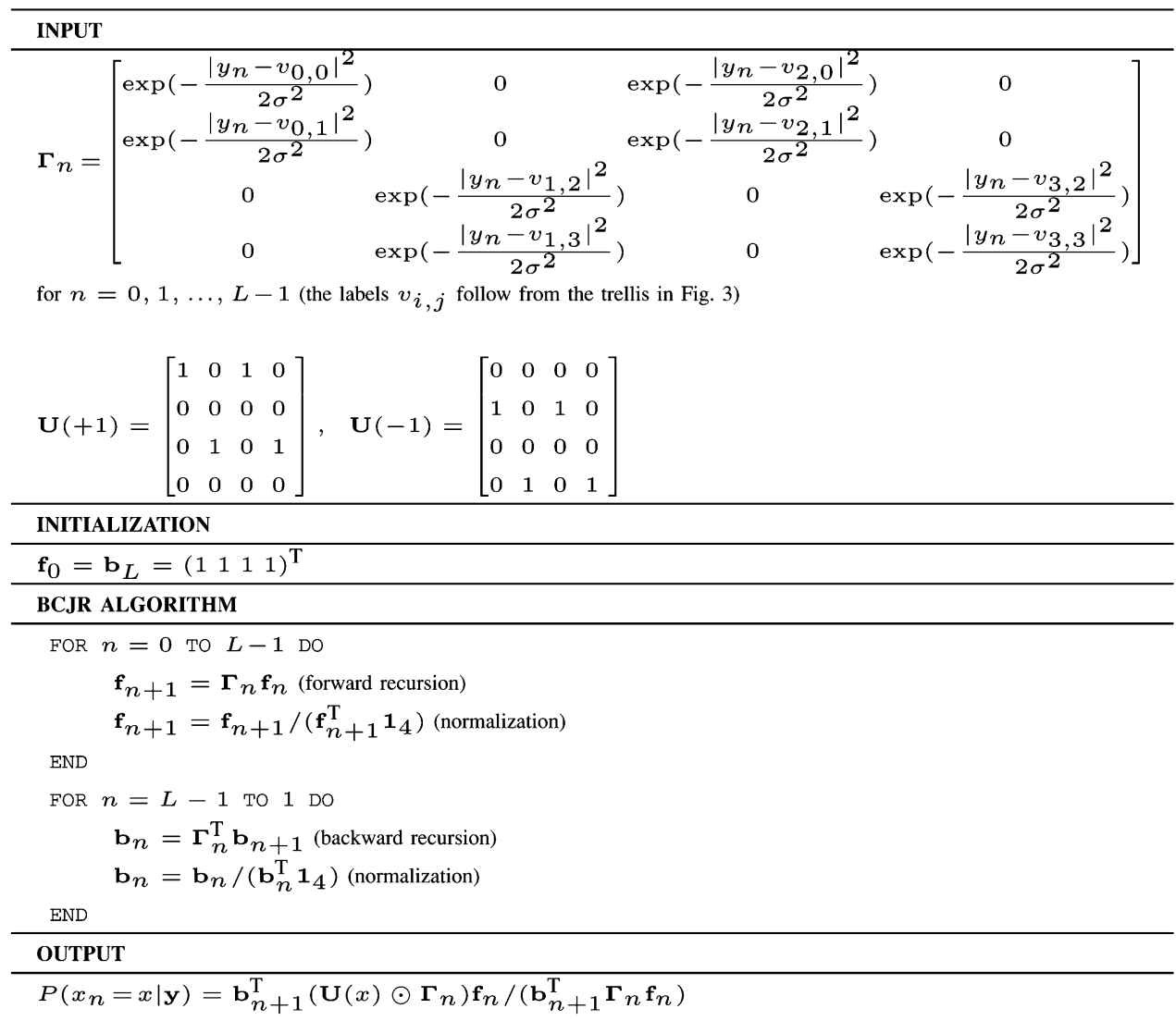

the difference between the two receiver approaches depicted in Fig. 4. A natural choice for the soft information $s\left(x_{n}\right)$ about the transmitted symbols $x_{n}$ are the posterior probabilities $P\left(x_{n} \mid \mathbf{y}\right)$, which are a by-product of the symbol-based MAP detector. Similarly, the less complex sequence-based MAP detector (Viterbi equalizer) can produce approximations of $P\left(x_{n} \mid \mathbf{y}\right)$ using, e.g., the soft-output Viterbi algorithm (SOVA) [31].

For filter-based methods, extracting soft information $s\left(x_{n}\right)$ is more involved. A common approach is to assume that the estimation error, $e_{n}=\hat{x}_{n}-x_{n}$, is Gaussian distributed [19], i.e., the $\operatorname{PDF} p\left(e_{n}\right)$ is given by $\mathcal{N}_{\mathbb{R}}\left(\mathrm{E}\left\{e_{n}\right\}, \operatorname{Var}\left\{e_{n}\right\}\right)$. For linear MMSE estimation, the mean and the covariance matrix of the sequence $\mathbf{e}=\left(e_{0} e_{1} \ldots e_{L-1}\right)^{\mathrm{T}}$ of estimation errors $e_{n}$ are given by

$$
\begin{aligned}
\mathrm{E}\{\mathbf{e}\} & =\mathbf{0}_{L}, \\
\operatorname{Cov}\{\mathbf{e}, \mathbf{e}\} & =\mathbf{I}_{L}-\mathbf{H}^{\mathrm{T}}\left(\sigma^{2} \mathbf{I}_{L}+\mathbf{H H}^{\mathrm{T}}\right)^{-1} \mathbf{H},
\end{aligned}
$$

The soft information $s\left(x_{n}\right)$ follows from sampling $p\left(e_{n}\right)$ at the values $e_{n}=\hat{x}_{n}-x$ for each $x \in \mathcal{S}$,

$$
s\left(x_{n}=x\right)=\kappa \cdot p\left(e_{n}\right) \text { for } e_{n}=\hat{x}_{n}-x,
$$

where the normalization constant $\kappa$ ensures that $\sum_{x \in \mathcal{S}} s\left(x_{n}=\right.$ $x)=1$. This scheme to compute soft information $s\left(x_{n}\right)$ from a filter output can be applied to other filter-based equalization algorithms as well.
Since the decoder in Fig. 4 operates on the code bits $c_{n}$, the next step for the receiver algorithm is to compute estimates $\hat{c}_{n}$ or soft information $s\left(c_{n}\right)$. The mapping from $|\mathcal{S}|=2^{q}$ probabilities $s\left(x_{n}\right)$ to $2 q$ probabilities $s\left(c_{n}\right)$ is commonly referred to as soft demapping. The demapping operation required here is quite simple, since $q=1$ and $\tilde{c}_{n}=c_{n}$ holds

$$
\begin{aligned}
& s\left(c_{n}=0\right)=s\left(x_{n}=+1\right), \\
& s\left(c_{n}=1\right)=s\left(x_{n}=-1\right) .
\end{aligned}
$$

After deinterleaving $\hat{c}_{n}$ to $\hat{b}_{n}$ or $s\left(c_{n}\right)$ to $s\left(b_{n}\right)$, respectively, the decoder can compute estimates $\hat{a}_{n}$ of the transmitted data bits.

The BEP-optimal MAP decoder given the soft information $s\left(b_{n}\right)$ is defined as follows:

$$
\hat{a}_{n}=\underset{a \in \mathbb{F}_{2}}{\operatorname{argmax}} P\left(a_{n}=a \mid s\left(b_{0}\right), s\left(b_{1}\right), \ldots, s\left(b_{N-1}\right)\right),
$$

with a slight abuse of notation, wherein the notation $P(x \mid s(y), s(z))$ is interpreted (here and in the sequel) as the distribution of $x$ when the variables $y$ and $z$ are assumed independent and distributed with the prior distributions $s(y)$ and $s(z)$, respectively. The posterior probabilities $P\left(a_{n} \mid s\left(b_{0}\right), s\left(b_{1}\right), \ldots, s\left(b_{N-1}\right)\right)$ can be computed efficiently using the BCJR algorithm as long as the FEC code has a trellis with a sufficiently small number of states. Table II applies the BCJR algorithm to the example code defined in Section II-A. 
TABLE II

Algorithm For COMPUTING THE POSTERIOR PROBABILITIEs $P\left(a_{n} \mid s\left(b_{0}\right), s\left(b_{1}\right), \ldots, s\left(b_{N-1}\right)\right)$ FOR THE MEMORY-2 CONVOLUTIONAL CODE DEFINED IN SECTION II-A

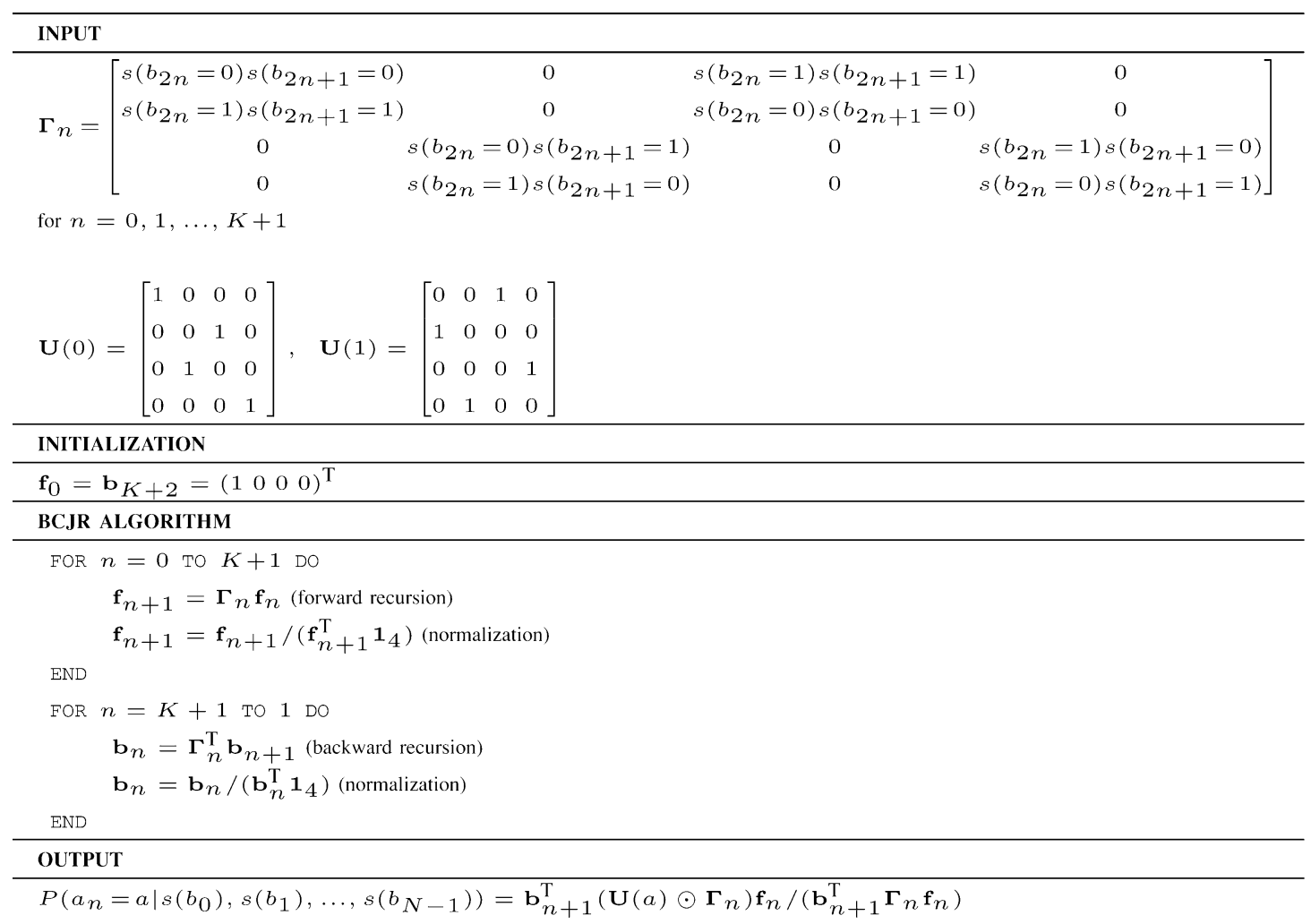

The entries $\gamma_{n}\left(s_{i}, s_{j}\right)$ of $\boldsymbol{\Gamma}_{n}$ and the initial vectors $\mathbf{f}_{0}$ and $\mathbf{b}_{K+2}$ follow from the code trellis in Fig. 2.

\section{Joint Equalization and Decoding}

A receiver algorithm where the equalizer is aware of the underlying code is often termed joint equalization and decoding. The exact implementation of this algorithm is intractable for a general code and interleaver, and as such, a feasible, yet suboptimal, alternative is typically sought.

Consider the following approach. Note first that the equalizer may apply the BCJR algorithm when $P(\mathbf{x})$ factors into $\prod_{k=0}^{L-1} P\left(x_{n}\right)$, which holds for independent symbols $x_{n}$. The symbol probabilities $P\left(x_{n}\right)$ can be different for each symbol value $x_{n} \in \mathcal{S}$ and each time step $n$. Initially, the equalizer imposes the IUD assumption on $x_{n}$ and sets $P\left(x_{n}\right)$ to $1 /|\mathcal{S}|$ for all $x_{n} \in \mathcal{S}$ while computing the soft information $s\left(x_{n}\right)$. The receiver algorithm continues by computing $s\left(c_{n}\right)$ and $s\left(b_{n}\right)$ followed by decoding. At this stage, the decoder may compute a new version of the soft information $s\left(b_{n}\right)$,

$$
s^{\prime}\left(b_{n}\right)=P\left(b_{n} \mid s\left(b_{0}\right), s\left(b_{1}\right), \ldots, s\left(b_{N-1}\right)\right) .
$$

The reliability of $s^{\prime}\left(b_{n}\right)$ compared to $s\left(b_{n}\right)$ generally improves because of the redundancy introduced during FEC encoding, i.e., the values in $s^{\prime}\left(b_{n}\right)$ are on average closer to 0 and 1 than those in $s\left(b_{n}\right)$. The posterior probabilities $s^{\prime}\left(b_{n}\right)$ can be computed with the symbol-based MAP decoder. In fact, to compute $s^{\prime}\left(b_{n}\right)$ for the example code from Section II-A, only the last line of the BCJR algorithm described in Table II need be updated to

$$
\begin{aligned}
s^{\prime}\left(b_{2 n}\right) & =P\left(b_{2 n}=b \mid s\left(b_{0}\right), s\left(b_{1}\right), \ldots, s\left(b_{N-1}\right)\right) \\
& =\mathbf{b}_{n+1}^{\mathrm{T}}\left(\mathbf{V}_{0}(b) \odot \boldsymbol{\Gamma}_{n}\right) \mathbf{f}_{n} /\left(\mathbf{b}_{n+1}^{\mathrm{T}} \boldsymbol{\Gamma}_{n} \mathbf{f}_{n}\right), \\
s^{\prime}\left(b_{2 n+1}\right) & =P\left(b_{2 n+1}=b \mid s\left(b_{0}\right), s\left(b_{1}\right), \ldots, s\left(b_{N-1}\right)\right) \\
& =\mathbf{b}_{n+1}^{\mathrm{T}}\left(\mathbf{V}_{1}(b) \odot \boldsymbol{\Gamma}_{n}\right) \mathbf{f}_{n} /\left(\mathbf{b}_{n+1}^{\mathrm{T}} \boldsymbol{\Gamma}_{n} \mathbf{f}_{n}\right),
\end{aligned}
$$

where

$$
\begin{aligned}
& \mathbf{V}_{0}(0)=\left[\begin{array}{llll}
1 & 0 & 0 & 0 \\
0 & 0 & 1 & 0 \\
0 & 1 & 0 & 0 \\
0 & 0 & 0 & 1
\end{array}\right], \\
& \mathbf{V}_{0}(1)=\left[\begin{array}{llll}
0 & 0 & 1 & 0 \\
1 & 0 & 0 & 0 \\
0 & 0 & 0 & 1 \\
0 & 1 & 0 & 0
\end{array}\right], \\
& \mathbf{V}_{1}(0)=\left[\begin{array}{llll}
1 & 0 & 0 & 0 \\
0 & 0 & 1 & 0 \\
0 & 0 & 0 & 1 \\
0 & 1 & 0 & 0
\end{array}\right], \\
& \mathbf{V}_{1}(1)=\left[\begin{array}{llll}
0 & 0 & 1 & 0 \\
1 & 0 & 0 & 0 \\
0 & 1 & 0 & 0 \\
0 & 0 & 0 & 1
\end{array}\right] .
\end{aligned}
$$

In this example, it turns out that $s\left(a_{n}\right)$ is equal to $s^{\prime}\left(b_{2 n}\right)$ or $\mathbf{U}(u)=\mathbf{V}_{0}(u)$, respectively, since the chosen encoding function is systematic. After interleaving $s^{\prime}\left(b_{n}\right)$ to $s^{\prime}\left(c_{n}\right)$, it makes 
sense to use the new soft information $s^{\prime}\left(c_{n}\right)$ to guide the equalizer with new symbol probabilities $s^{\prime}\left(x_{n}\right)$ or soft information, respectively, given the improved knowledge about the bits $c_{n}$. The mapping from $2 N$ probabilities $s^{\prime}\left(c_{n}\right)$ to $L 2^{q}$ probabilities $s^{\prime}\left(x_{n}\right)$ given a $2^{q}$-ary signal alphabet $\mathcal{S}$ is commonly referred to as soft mapping. The soft mapping required here is quite simple: $s^{\prime}\left(x_{n}=+1\right)=s^{\prime}\left(c_{n}=0\right)$ and $s^{\prime}\left(x_{n}=-1\right)=s^{\prime}\left(c_{n}=1\right)$.

With the soft information $s^{\prime}\left(x_{n}\right)$ at hand, it is natural to repeat the equalization step, yielding updated soft information $s\left(x_{n}\right)$. Incorporating $s^{\prime}\left(x_{n}\right)$ into the trellis-based equalization algorithms (symbol- or sequence-based MAP detection) is straightforward. Only the transition matrices $\boldsymbol{\Gamma}_{n}$ in the initialization step of the BCJR algorithm described in Table I need be updated as shown in the first equation at the bottom of the page,for $i, j=0,1,2,3$, where $v_{i, j}$ and $x_{i, j}$ follow from the trellis in Fig. 3 (bottom trellis), e.g., $v_{0,0}=1.63$ and $x_{0,0}=+1$. Also the linear MMSE estimator (8) can take advantage of the probabilities $s^{\prime}\left(x_{n}\right)$ by recomputing the symbol statistics $\mathrm{E}\left\{x_{n}\right\}$ and $\operatorname{Cov}\left\{x_{n}, x_{n}\right\}$. This approach is derived and analyzed in detail in Section II-E.

The updated probabilities $s\left(x_{n}\right)$ are again soft demapped to $s\left(c_{n}\right)$ followed by deinterleaving and decoding. This amounts to (suboptimal) joint equalization and decoding, since the equalizer incorporates knowledge about the underlying code. The result is an iterative receiver algorithm, which recomputes the soft information $s\left(x_{n}\right), s\left(c_{n}\right), s\left(b_{n}\right)$ (equalizer to decoder) and $s^{\prime}\left(b_{n}\right), s^{\prime}\left(c_{n}\right), s^{\prime}\left(x_{n}\right)$ (decoder to equalizer) by iterating equalization and decoding tasks, passing soft information between them. The BER performance of the receiver indeed improves with the iterations, but not significantly. In contrast, and perhaps best motivated by the factorization of the a posteriori probability as might be depicted using a factor graph, the improvement can be tremendous when the following information is fed back from the decoder to the equalizer:

$$
\begin{aligned}
& s_{\text {ext }}^{\prime}\left(b_{n}\right)=P\left(b_{n} \mid s\left(b_{0}\right), s\left(b_{1}\right), \ldots,\right. \\
& \left.\quad s\left(b_{n-1}\right), s\left(b_{n+1}\right), \ldots, s\left(b_{N-1}\right)\right) .
\end{aligned}
$$

This can be viewed as the corresponding message that would be sent into the variable node in the corresponding factor graph. This quantity is the extrinsic soft information about $b_{n}$ contained in $s\left(b_{0}\right), s\left(b_{1}\right), \ldots s\left(b_{N-1}\right)$ except $s\left(b_{n}\right)$. Similarly, the equalizer should communicate extrinsic soft information $s_{\text {ext }}\left(c_{n}\right)$ to the decoder computed using the observation $y$ and all $s_{\text {ext }}^{\prime}\left(c_{0}\right), s_{\text {ext }}^{\prime}\left(c_{1}\right), \ldots, s_{\text {ext }}^{\prime}\left(c_{N-1}\right)$ except $s_{\text {ext }}^{\prime}\left(c_{n}\right)$. This approach to passing extrinsic soft information between constituent algorithms was first proposed by [10] in the context of decoding turbo codes and has been extended to various concatenated communication systems such as coded data transmission over ISI channels [2], [27], [29], [39], where it is called turbo equalization.

Since the code bits $b_{n}$ are from a binary alphabet, it is often more convenient to replace the two probabilities $s\left(b_{n}=0\right)$ and $s\left(b_{n}=1\right)$ by the LLR

$$
\lambda\left(b_{n}\right)=\ln \frac{s\left(b_{n}=0\right)}{s\left(b_{n}=1\right)} .
$$

Using the LLRs $\lambda\left(b_{n}\right)$ for decoding seems to be counterintuitive, since they must be incorporated into the matrices $\boldsymbol{\Gamma}_{n}$ in Table II, which requires several exponentiation operations. However, when implementing the BCJR algorithm with the logarithms of $\mathbf{f}_{n}, \mathbf{b}_{n}$, and $\boldsymbol{\Gamma}_{n}$, it is much easier to use $\lambda\left(b_{n}\right)$ in $\ln \boldsymbol{\Gamma}_{n}$ [12], [81]. Consequently, instead of the extrinsic soft information $s_{\text {ext }}^{\prime}\left(b_{n}\right)$, it is also more practical to consider the extrinsic LLR (see second equation at the bottom of the page). If the soft information $s^{\prime}\left(b_{n}\right)$ is a posterior probability, i.e., $s^{\prime}\left(b_{n}\right)=P\left(b_{n} \mid s\left(b_{0}\right), s\left(b_{1}\right), \ldots, s\left(b_{N-1}\right)\right)$, it is possible to apply the decomposition (7) to the LLR $\lambda^{\prime}\left(b_{n}\right)$ corresponding to $s^{\prime}\left(b_{n}\right)$

$$
\begin{aligned}
\lambda_{\text {ext }}^{\prime}\left(b_{n}\right)= & \ln \frac{P\left(b_{n}=0 \mid s\left(b_{0}\right), s\left(b_{1}\right), \ldots, s\left(b_{N-1}\right)\right)}{P\left(b_{n}=1 \mid s\left(b_{0}\right), s\left(b_{1}\right), \ldots, s\left(b_{N-1}\right)\right)} \\
& -\ln \frac{s\left(b_{n}=0\right)}{s\left(b_{n}=0\right)}, \\
= & \underbrace{\ln \frac{s^{\prime}\left(b_{n}=0\right)}{s^{\prime}\left(b_{n}=1\right)}}-\underbrace{\ln \frac{s\left(b_{n}=0\right)}{s\left(b_{n}=0\right)}}, \\
= & \lambda^{\prime}\left(b_{n}\right)-\lambda\left(b_{n}\right) .
\end{aligned}
$$

For example, the extrinsic LLRs $\lambda_{\text {ext }}^{\prime}\left(b_{n}\right)$ for the memory-2 convolutional code defined in Section II-A can be computed using the BCJR algorithm described in Table II as follows:

$$
\begin{aligned}
\lambda_{\text {ext }}^{\prime}\left(b_{2 n+i}\right)=\ln \frac{\mathbf{b}_{n+1}^{\mathrm{T}}\left(\mathbf{V}_{i}(0) \odot \boldsymbol{\Gamma}_{n}\right) \mathbf{f}_{n}}{\mathbf{b}_{n+1}^{\mathrm{T}}\left(\mathbf{V}_{i}(1) \odot \boldsymbol{\Gamma}_{n}\right) \mathbf{f}_{n}}-\lambda\left(b_{2 n}\right), \\
i=0,1, \quad n=0,1, \ldots, K+1 .
\end{aligned}
$$

However, this formula may produce incorrect extrinsic LLRs $\lambda_{\text {ext }}^{\prime}\left(b_{n}\right)$ in a practical implementation due to numerical prob-

$$
\boldsymbol{\Gamma}_{n}[j, i]= \begin{cases}\exp \left(-\frac{\left|y_{n}-v_{i, j}\right|^{2}}{2 \sigma^{2}}\right) s^{\prime}\left(x_{n}=x_{i, j}\right), & (i j) \text { corresponds to a valid trellis branch } \\ 0, & \text { otherwise }\end{cases}
$$

$$
\lambda_{\text {ext }}^{\prime}\left(b_{n}\right)=\ln \frac{s_{\text {ext }}^{\prime}\left(b_{n}=0\right)}{s_{\text {ext }}^{\prime}\left(b_{n}=1\right)}=\ln \frac{P\left(b_{n}=0 \mid s\left(b_{0}\right), s\left(b_{1}\right), \ldots, s\left(b_{n-1}\right), s\left(b_{n+1}\right), \ldots, s\left(b_{N-1}\right)\right)}{P\left(b_{n}=1 \mid s\left(b_{0}\right), s\left(b_{1}\right), \ldots, s\left(b_{n-1}\right), s\left(b_{n+1}\right), \ldots, s\left(b_{N-1}\right)\right)}
$$




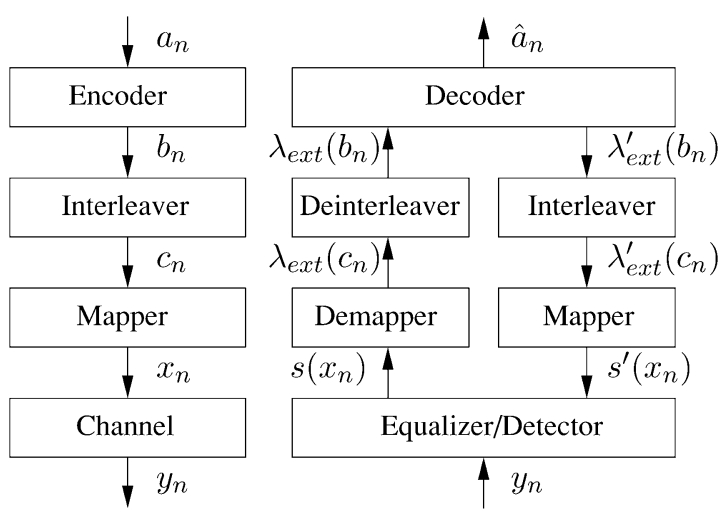

\begin{tabular}{ll}
\hline \multicolumn{1}{c}{ Turbo equalization } \\
\hline (1) & initialization: set $\lambda_{\text {ext }}^{\prime}\left(c_{n}\right)$ to 0 for all $n=0,1, \ldots, N-1$ \\
(2) & equalization: compute $\lambda_{\text {ext }}\left(c_{n}\right)$ for all $n$ from $\mathbf{y}$ \\
& and $\lambda_{\text {ext }}^{\prime}\left(c_{0}\right), \ldots, \lambda_{\text {ext }}^{\prime}\left(c_{N-1}\right)$ except $\lambda_{\text {ext }}^{\prime}\left(c_{n}\right)$ \\
(3) deinterleaving: permute $\lambda_{\text {ext }}\left(c_{n}\right)$ to $\lambda_{\text {ext }}\left(b_{n}\right)$ for all $n$ \\
(4) termination: subject to a suitable termination criterion, \\
\\
go to (5) or compute $\hat{a}_{n}$ for all $n=0,1, \ldots, K-1$ from \\
$\lambda_{\text {ext }}\left(b_{0}\right), \ldots, \lambda_{\text {ext }}\left(b_{N-1}\right)$ and stop \\
(5) decoding: compute $\lambda_{\text {ext }}^{\prime}\left(b_{n}\right)$ for all $n$ from \\
\\
$\lambda_{\text {ext }}\left(b_{0}\right), \ldots, \lambda_{\text {ext }}\left(b_{N-1}\right)$ except $\lambda_{\text {ext }}\left(b_{n}\right)$ \\
(6) interleaving: permute $\lambda_{\text {ext }}^{\prime}\left(b_{n}\right)$ to $\lambda_{\text {ext }}^{\prime}\left(c_{n}\right)$ for all $n$ \\
(7) go to (2)
\end{tabular}

Fig. 5. Receiver structure and basic steps of the turbo equalization algorithm.

lems in computing the difference $\lambda^{\prime}\left(b_{n}\right)-\lambda\left(b_{n}\right)$. Instead, $\lambda_{\text {ext }}^{\prime}\left(b_{n}\right)$ should be computed directly as follows:

$$
\lambda_{\text {ext }}^{\prime}\left(b_{2 n+i}\right)=\ln \frac{\mathbf{b}_{n+1}^{\mathrm{T}}\left(\mathbf{V}_{i}(0) \odot \boldsymbol{\Gamma}_{\mathrm{ext}, i, n}\right) \mathbf{f}_{n}}{\mathbf{b}_{n+1}^{\mathrm{T}}\left(\mathbf{V}_{i}(1) \odot \boldsymbol{\Gamma}_{\mathrm{ext}, i, n}\right) \mathbf{f}_{n}}
$$

where the $\boldsymbol{\Gamma}_{\text {ext, }, i, n}$ are extrinsic transition matrices, which do not depend on the input LLR $\lambda_{\text {ext }}\left(b_{2 n+i}\right)$ while computing $\lambda_{\text {ext }}^{\prime}\left(b_{2 n+i}\right)$. For example, the extrinsic transition matrix $\boldsymbol{\Gamma}_{\text {ext }, 0, n}$ corresponding to $\lambda_{\text {ext }}^{\prime}\left(b_{2 n}\right)$ for the memory- 2 convolutional code defined in Section II-A is given by the equation at the bottom of the page. Similarly, the extrinsic LLR $\lambda_{\text {ext }}\left(c_{n}\right)$ is defined corresponding to $s_{\text {ext }}\left(c_{n}\right)$

$$
\lambda_{\text {ext }}\left(c_{n}\right)=\ln \frac{s_{\text {ext }}\left(c_{n}=0\right)}{s_{\text {ext }}\left(c_{n}=1\right)} .
$$

For example, the extrinsic LLRs $\lambda_{\text {ext }}\left(c_{n}\right)$ for the ISI channel model defined in Section II-A can be computed using the BCJR algorithm described in Table II as follows:

$$
\begin{aligned}
\lambda_{\text {ext }}\left(c_{n}\right) & =\left.\ln \frac{s\left(x_{n}=+1\right)}{s\left(x_{n}=-1\right)}\right|_{\text {without using } \lambda_{\text {ext }}^{\prime}\left(c_{n}\right)} \\
& =\left.\ln \frac{P\left(x_{n}=+1 \mid \mathbf{y}\right)}{P\left(x_{n}=-1 \mid \mathbf{y}\right)}\right|_{\text {without using } \lambda_{\text {ext }}^{\prime}\left(c_{n}\right)} \\
& =\left.\ln \frac{\mathbf{b}_{n+1}^{\mathrm{T}}\left(\mathbf{U}(+1) \odot \boldsymbol{\Gamma}_{n}\right) \mathbf{f}_{n}}{\mathbf{b}_{n+1}^{\mathrm{T}}\left(\mathbf{U}(-1) \odot \boldsymbol{\Gamma}_{n}\right) \mathbf{f}_{n}}\right|_{\text {without using } \lambda_{\text {ext }}^{\prime}\left(c_{n}\right)} \\
& =\ln \frac{\mathbf{b}_{n+1}^{\mathrm{T}}\left(\mathbf{U}(+1) \odot \boldsymbol{\Gamma}_{n}\right) \mathbf{f}_{n}}{\mathbf{b}_{n+1}^{\mathrm{T}}\left(\mathbf{U}(-1) \odot \boldsymbol{\Gamma}_{n}\right) \mathbf{f}_{n}}-\lambda_{\text {ext }}^{\prime}\left(c_{n}\right) .
\end{aligned}
$$

The last line follows from using that $s_{\text {ext }}^{\prime}\left(c_{n}=0\right)$ can be factored out of $\mathbf{b}_{n+1}^{\mathrm{T}}\left(\mathbf{U}(+1) \odot \boldsymbol{\Gamma}_{n}\right) \mathbf{f}_{n}$ and that $s_{\text {ext }}^{\prime}\left(c_{n}=1\right)$ can be factored out of $\mathbf{b}_{n+1}^{\mathrm{T}}\left(\mathbf{U}(-1) \odot \boldsymbol{\Gamma}_{n}\right) \mathbf{f}_{n}$. However, this relationship holds only for symbol-based MAP detection. In a practical implementation, $\lambda_{\text {ext }}\left(c_{n}\right)$ should be computed directly with an extrinsic transition matrix $\boldsymbol{\Gamma}_{\text {ext }, n}$ to avoid numerical problems

$$
\lambda_{\text {ext }}\left(c_{n}\right)=\ln \frac{\mathbf{b}_{n+1}^{\mathrm{T}}\left(\mathbf{U}(+1) \odot \boldsymbol{\Gamma}_{\text {ext }, n}\right) \mathbf{f}_{n}}{\mathbf{b}_{n+1}^{\mathrm{T}}\left(\mathbf{U}(-1) \odot \boldsymbol{\Gamma}_{\mathrm{ext}, n}\right) \mathbf{f}_{n}},
$$

where $\boldsymbol{\Gamma}_{\text {ext }, n}[j, i]=\exp \left(-\left|y_{n}-v_{i, j}\right|^{2} /\left(2 \sigma^{2}\right)\right)$ if $(i j)$ corresponds to a valid trellis branch and $\boldsymbol{\Gamma}_{\text {ext }, n}[j, i]=0$ otherwise. The basic steps of turbo equalization are summarized in Fig. 5 .

The BER performance of turbo equalization using symbolbased MAP detection as an equalization algorithm is exhibited in Fig. 6 for block length $K=510$. Using the equalizer and the decoder once corresponds to separate equalization and decoding. Applying the steps outlined in Fig. 5 results in a performance gain. After one additional equalization-decoding step, a so-called iteration, more than $3 \mathrm{~dB}$ SNR $P / \sigma^{2}$ are gained at a BER of $10^{-5}$. More iterations do not improve the performance significantly and instead, a limit seems to exist, which is identical to the BER performance of the soft decoder (10) without ISI in the channel (dotted line in Fig. 6). In this case, the channel model (1) simplifies to $y_{n}=x_{n}+w_{n}$ such that the extrinsic LLRs $\lambda_{\text {ext }}\left(c_{n}\right)$ are given by

$$
\begin{aligned}
& \lambda_{\text {ext }}\left(c_{n}\right) \\
& \quad=\left.\ln \frac{P\left(x_{n}=+1 \mid y_{n}\right)}{P\left(x_{n}=-1 \mid y_{n}\right)}\right|_{\text {without using } \lambda_{\text {ext }}^{\prime}\left(c_{n}\right)} \\
& \quad=\left.\ln \frac{p\left(y_{n} \mid x_{n}=+1\right) s^{\prime}\left(x_{n}=+1\right)}{p\left(y_{n} \mid x_{n}=-1\right) s^{\prime}\left(x_{n}=-1\right)}\right|_{\text {without using } \lambda_{\text {ext }}^{\prime}\left(c_{n}\right)} .
\end{aligned}
$$

$$
\boldsymbol{\Gamma}_{\text {ext }, 0, n}=\left[\begin{array}{cccc}
s\left(b_{2 n+1}=0\right) & 0 & s\left(b_{2 n+1}=1\right) & 0 \\
s\left(b_{2 n+1}=1\right) & 0 & s\left(b_{2 n+1}=0\right) & 0 \\
0 & s\left(b_{2 n+1}=1\right) & 0 & s\left(b_{2 n+1}=0\right) \\
0 & s\left(b_{2 n+1}=0\right) & 0 & s\left(b_{2 n+1}=1\right)
\end{array}\right]
$$




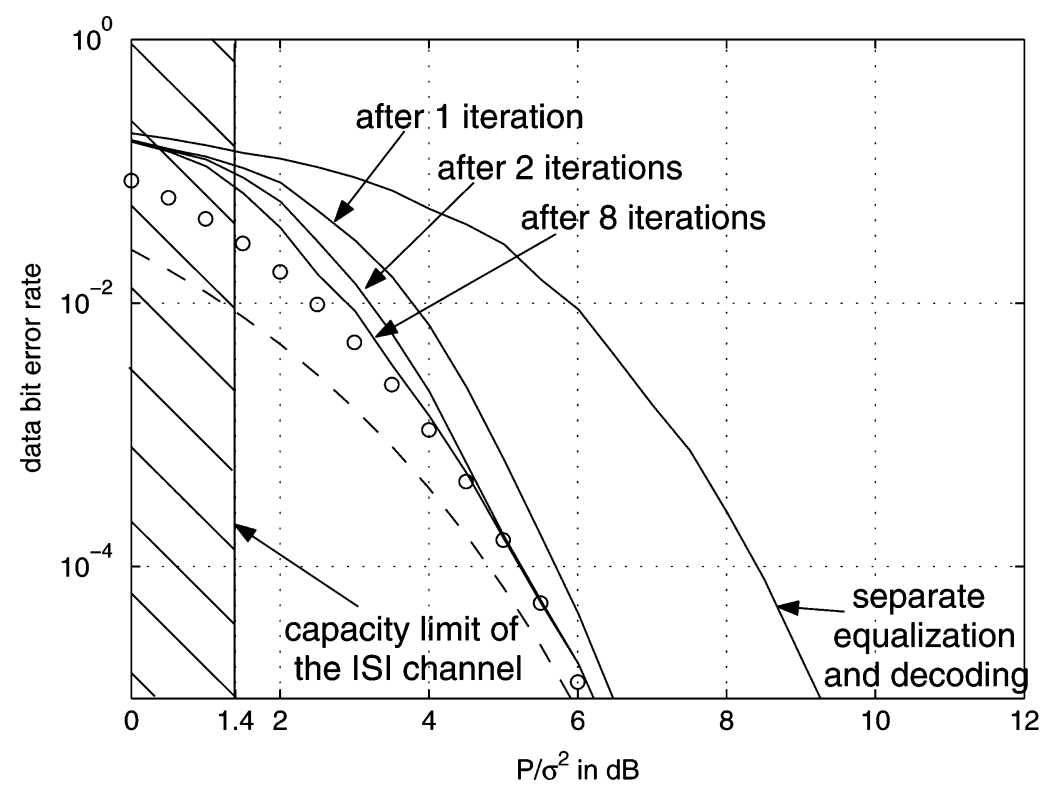

Fig. 6. Performance of turbo equalization for the communication system described in Section II-A using symbol-based MAP detection. The BER performance (solid lines) is plotted for separate equalization and decoding as well as after one, two, or eight iterations. The dotted line corresponds to the BER performance of the FEC decoder when no ISI is introduced in the channel. The dashed line is a lower bound on the BER performance of any decoder. The considered blocklength is $K=510(N=L=1024)$. An S-random interleaver with $S=16$ is applied.

The PDF $p\left(y_{n} \mid x_{n}\right)$ is equal to $\mathcal{N}_{\mathbb{R}}\left(x_{n}, \sigma^{2}\right)$. However, $s^{\prime}\left(x_{n}\right)$ follows directly from $\lambda_{\text {ext }}^{\prime}\left(c_{n}\right)$ and must be discarded while computing $\lambda_{\text {ext }}\left(c_{n}\right)$, which yields

$$
\begin{aligned}
\lambda_{\text {ext }}\left(c_{n}\right) & =\ln \frac{p\left(y_{n} \mid x_{n}=+1\right)}{p\left(y_{n} \mid x_{n}=-1\right)} \\
& =\ln \frac{\exp \left(-\left(y_{n}-+1\right)^{2} /\left(2 \sigma^{2}\right)\right)}{\exp \left(-\left(y_{n}--1\right)^{2} /\left(2 \sigma^{2}\right)\right)}=\frac{2 y_{n}}{\sigma^{2}} .
\end{aligned}
$$

As such, performing several equalization-decoding tasks (iterations) given an ISI-free channel does not improve the LLRs $\lambda_{\text {ext }}\left(c_{n}\right)$, i.e., a single decoding task is sufficient to achieve the BER performance depicted in Fig. 6. This finding does not hold for higher-order signal alphabets as shown in Section III.

The lower bound on the BER performance, derived from properties of the ISI channel and the code, also shows that the communication system defined in Section II-A is not well designed, since even the best possible decoder is still $4.5 \mathrm{~dB}$ SNR $P / \sigma^{2}$ away from the channel capacity at a BER of $10^{-5}$. A remedy to this problem could be to increase the minimum distance $d_{\min , \text { fec }}$ of the convolutional code, but this results in a decoding complexity increase. Using precoding is a more elegant way to improve the distance spectrum. Such communication systems together with a turbo equalization receiver are studied in Section V.

It is interesting to consider the block length $K$ required to approach the performance of the BEP optimal receiver, or whether alternative approaches to turbo equalization exist with better performance. These questions are hard to answer and the graphical descriptions of coded data transmission over an ISI channel introduced in [85], [86] suggest other iterative receiver approaches that may perform as well as turbo equalization (or even better).

The distance spectrum of the overall code, generated by the concatenation of the outer code with the inner rate-one code through an interleaver, yields the lower bound (dashed line) in Fig. 6. It also decorrelates error bursts or, say, dependencies between neighboring samples of the soft information $\lambda_{\text {ext }}\left(c_{n}\right)$ and $\lambda_{\text {ext }}^{\prime}\left(b_{n}\right)$ produced by the equalizer and the decoder, respectively. Recall that the BCJR algorithm applied for detection and decoding assumes that the input symbols to the state-space model are independent, i.e., the input symbols $x_{n}$ for the ISI channel as well as the soft information $s^{\prime}\left(x_{n}\right)$ about them are assumed independent. The same holds for the probability information about the output of a state-space model, i.e., the soft information $\lambda\left(b_{n}\right)$ about the code bits $b_{n}$ must be independent. The interleaver cannot remove dependencies in between the permuted bits or symbols, but it can reduce local dependencies.

The minimal required $\mathrm{S}$ parameter of the interleaver in a communication system applying turbo equalization can vary tremendously depending on the system configuration. For example, large memory in the channel or the FEC code usually requires a larger $\mathrm{S}$ and, thus, a larger block length for turbo equalization to work effectively.

Another issue of interest is the number of required iterations to achieve desirable BER performance. As a practical rule, longer block lengths require more iterations to approach the performance of the BEP-optimal decoder. In a practical implementation, the number of iterations is often constrained by the computational complexity allowed and the delay of the receiver algorithm. For more information about this topic, the reader is referred to [11], [79], and [87].

\section{E. SISO Equalization Based on Linear MMSE Estimation}

Fig. 7 shows how the linear MMSE estimator introduced in Section II-C can be integrated into the turbo equalization setup defined in Fig. 5. It is called a soft-in soft-out (SISO) equalizer because it outputs and processes soft information. Clearly, the symbol-based MAP detector used in the previous section is a SISO equalizer as well. 


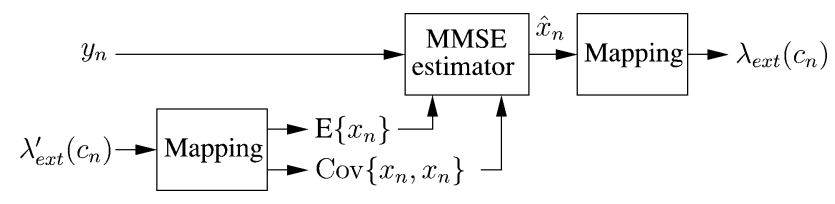

Fig. 7. A SISO equalizer based on linear MMSE estimation.

The first step is to incorporate the extrinsic LLRs $\lambda_{\text {ext }}^{\prime}\left(c_{n}\right)$ into the equalization process. This is done by mapping the LLRs $\lambda_{\text {ext }}^{\prime}\left(c_{n}\right)$ to probabilities $s^{\prime}\left(x_{n}\right)$ followed by mapping them to new statistics $\mu_{n}=\mathrm{E}\left\{x_{n}\right\}$ and $v_{n}=\operatorname{Cov}\left\{x_{n}, x_{n}\right\}$ of the symbols $x_{n}$. For BPSK modulation, this mapping is as follows:

$$
\begin{aligned}
\mu_{n} & =\sum_{x \in \mathcal{S}} x \cdot s^{\prime}\left(x_{n}=x\right)=s^{\prime}\left(x_{n}=+1\right)-s^{\prime}\left(x_{n}=-1\right) \\
& =s^{\prime}\left(c_{n}=0\right)-s^{\prime}\left(c_{n}=1\right) \\
& =\frac{1}{1+e^{-\lambda_{\text {ext }}^{\prime}\left(c_{n}\right)}}-\frac{e^{-\lambda_{\text {ext }}^{\prime}\left(c_{n}\right)}}{1+e^{-\lambda_{\text {ext }}^{\prime}\left(c_{n}\right)}}=\tanh \left(\lambda_{\text {ext }}^{\prime}\left(c_{n}\right) / 2\right), \\
v_{n} & =\sum_{x \in \mathcal{S}}\left|x-\mathrm{E}\left\{x_{n}\right\}\right|^{2} \cdot s^{\prime}\left(x_{n}=x\right)=1-\left|\mu_{n}\right|^{2} .
\end{aligned}
$$

A second step is to map the symbol estimates $\hat{x}_{n}$ produced from the linear MMSE estimator to the extrinsic LLRs $\lambda_{\text {ext }}\left(c_{n}\right)$. Combining (9) with (11) yields the following rule to compute $\lambda_{\text {ext }}\left(c_{n}\right)$ :

$$
\begin{aligned}
\lambda_{\text {ext }}\left(c_{n}\right) & =\ln \frac{s_{\text {ext }}\left(c_{n}=0\right)}{s_{\text {ext }}\left(c_{n}=1\right)} \\
& =\left.\ln \frac{s\left(x_{n}=+1\right)}{s\left(x_{n}=-1\right)}\right|_{\text {without using } \lambda_{\text {ext }}^{\prime}\left(c_{n}\right)},
\end{aligned}
$$

where $s\left(x_{n}=x\right)$ is the value of the PDF $p\left(e_{n}\right)$ evaluated at $e_{n}=\hat{x}_{n}-x$. The extrinsic LLR $\lambda_{\text {ext }}\left(c_{n}\right)$ should not depend on $\lambda_{\text {ext }}^{\prime}\left(c_{n}\right)$ and, consequently, neither should the estimate $\hat{x}_{n}$ depend on $\lambda_{\text {ext }}^{\prime}\left(c_{n}\right)$, which affects the derivation of the estimation algorithm.

In contrast to Section II-C, the linear MMSE estimator considered here processes a length- $W$ window of observations $\mathbf{y}_{n}=\left(y_{n-W_{2}} y_{n-W_{2}+1} \ldots y_{n+W_{1}}\right)^{T}, W=W_{1}+W_{2}+1$, rather than the complete sequence $\mathbf{y}$ to compute the estimate $\hat{x}_{n}$. The solution in (8) produces all estimates $\hat{x}_{n}$ at once, but this requires the solution of an $L \times L$ system of equations (complexity $O\left(L^{3}\right)$ ) and to linearly process the sequence $\mathbf{y}$ (complexity $O\left(L^{2}\right)$ ). In the remainder of this section, the system matrix $\mathbf{H}$ is constructed under the termination assumption as shown on the left side of (2).
The estimate $\hat{x}_{n}$ of $x_{n}$ minimizing the $\operatorname{MSE} \mathrm{E}\left\{\left|\hat{x}_{n}-x_{n}\right|^{2}\right\}$ is given by

$$
\begin{aligned}
\hat{x}_{n}=\mathrm{E}\left\{x_{n}\right\} & \\
& +\operatorname{Cov}\left\{x_{n}, \mathbf{y}_{n}\right\} \operatorname{Cov}\left\{\mathbf{y}_{n}, \mathbf{y}_{n}\right\}^{-1}\left(\mathbf{y}_{n}-\mathrm{E}\left\{\mathbf{y}_{n}\right\}\right) .
\end{aligned}
$$

From the ISI channel model (1) it follows that (14) is given by

$$
\begin{aligned}
\hat{x}_{n} & =\mu_{n}+v_{n} \mathbf{h}_{n}^{\mathrm{T}} \boldsymbol{\Sigma}_{n}^{-1}\left(\mathbf{y}_{n}-\mathbf{H}_{n} \boldsymbol{\mu}_{n}\right), \\
\boldsymbol{\Sigma}_{n} & =\sigma^{2} \mathbf{I}_{W}+\mathbf{H}_{n} \mathbf{V}_{n} \mathbf{H}_{n}^{\mathrm{T}},
\end{aligned}
$$

where $\mathbf{H}_{n}$ is the $W \times\left(W+M_{h}\right)$ submatrix $\mathbf{H}\left[n-W_{2}: n+\right.$ $\left.W_{1}, n-W_{2}-M_{h}: n+W_{1}\right]$ of the system matrix $\mathbf{H}$ and

$$
\begin{aligned}
\boldsymbol{\mu}_{n} & =\left(\mu_{n-M_{h}-W_{2}} \mu_{n-M_{h}-W_{2}+1} \ldots \mu_{n+W_{1}}\right)^{\mathrm{T}}, \\
\mathbf{V}_{n} & =\operatorname{Diag}\left\{v_{n-M_{h}-W_{2}} v_{n-M_{h}-W_{2}+1} \ldots v_{n+W_{1}}\right\}, \\
\mathbf{h}_{n} & =\mathbf{H}_{n}\left[0: W-1, W_{2}+M_{h}\right],
\end{aligned}
$$

i.e., $\mathbf{h}_{n}$ is the $\left(W_{2}+M_{h}\right)$ th column of $\mathbf{H}_{n}$. The submatrix $\mathbf{H}_{n}$ is given by the equation at the bottom of the page for the time indices $n=W_{2}, W_{2}+1 \ldots, L-W_{1}-1$. For simplicity, $\mathbf{H}_{n}$ is assumed to have the same structure for the remaining time indices. Although $\mathbf{H}_{n}$ is time-invariant, the time index is kept to make clear that the following equalization algorithms apply to time-varying ISI channels as well.

Under the termination assumption, the statistics $\mu_{n}$ and $v_{n}$ are set to 0 and 1 , respectively, for time indices $n$ outside the range $0,1, \ldots, L-1$, which assumes that no information about the corresponding symbol $x_{n}$ is available and the IUD assumption is applied. The analogous conditions are treated in a straightforward manner.

The estimate $\hat{x}_{n}$ calculated in (15) depends on $\lambda_{\text {ext }}^{\prime}\left(c_{n}\right)$ via $\mu_{n}$ and $v_{n}$. In order that $\hat{x}_{n}$ be independent from $\lambda_{\text {ext }}^{\prime}\left(c_{n}\right)$, this particular LLR is set to 0 while computing $\hat{x}_{n}$. This choice corresponds to an IUD assumption made on the particular symbol $x_{n}$. It follows that $\mu_{n}$ and $v_{n}$ should be replaced by 0 and 1 , respectively, while computing $\hat{x}_{n}$, which changes (15) to

$$
\begin{aligned}
\hat{x}_{n}=\mathbf{h}_{n}^{\mathrm{T}}\left(\boldsymbol{\Sigma}_{n}+\left(1-v_{n}\right) \mathbf{h}_{n} \mathbf{h}_{n}^{\mathrm{T}}\right)^{-1} & \\
& \times\left(\mathbf{y}_{n}-\mathbf{H}_{n} \boldsymbol{\mu}_{n}+\mu_{n} \mathbf{h}_{n}\right) .
\end{aligned}
$$

Using Woodbury's identity [88], the expression $\mathbf{h}_{n}^{\mathrm{T}}\left(\boldsymbol{\Sigma}_{n}+(1-\right.$ $\left.\left.v_{n}\right) \mathbf{h}_{n} \mathbf{h}_{n}^{\mathrm{T}}\right)^{-1}$ can be simplified as follows:

$$
\begin{aligned}
\mathbf{h}_{n}^{\mathrm{T}} & \left(\boldsymbol{\Sigma}_{n}+\left(1-v_{n}\right) \mathbf{h}_{n} \mathbf{h}_{n}^{\mathrm{T}}\right)^{-1} \\
= & \mathbf{h}_{n}^{\mathrm{T}}\left(\boldsymbol{\Sigma}_{n}^{-1}-\boldsymbol{\Sigma}_{n}^{-1} \mathbf{h}_{n} \mathbf{h}_{n}^{\mathrm{T}} \boldsymbol{\Sigma}_{n}^{-1} /\left(\left(1-v_{n}\right)^{-1}\right.\right. \\
& \left.+\mathbf{h}_{n}^{\mathrm{T}} \boldsymbol{\Sigma}_{n}^{-1} \mathbf{h}_{n}\right) \\
= & \mathbf{f}_{n}^{\mathrm{T}}-\left(\mathbf{f}_{n}^{\mathrm{T}} \mathbf{h}_{n}\right) \cdot \mathbf{f}_{n}^{\mathrm{T}} /\left(\left(1-v_{n}\right)^{-1}+\mathbf{f}_{n}^{\mathrm{T}} \mathbf{h}_{n}\right) \quad \text { with }
\end{aligned}
$$

$$
\mathbf{H}_{n}=\left[\begin{array}{cccccccc}
h_{M_{h}} & h_{M_{h}-1} & \ldots & h_{0} & 0 & \cdots & & 0 \\
0 & h_{M_{h}} & h_{M_{h}-1} & \cdots & h_{0} & 0 & \cdots & 0 \\
& & & \ddots & & & & \\
0 & & \ldots & 0 & h_{M_{h}} & h_{M_{h}-1} & \cdots & h_{0}
\end{array}\right]
$$


TABLE III

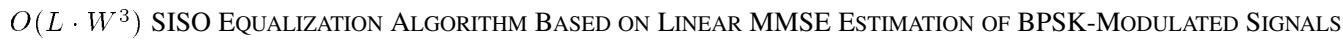

\begin{tabular}{l}
$\frac{\text { INPUT }}{\text { extrinsic LLRs } \lambda_{e x t}^{\prime}\left(c_{n}\right) \text { for } n=0,1, \ldots, N-1}$ \\
$\frac{\text { INITIALIZATION }}{\text { compute } \mu_{n}=\tanh \left(\lambda_{e x t}^{\prime}\left(c_{n}\right) / 2\right) \text { and } v_{n}=1-\left|\mu_{n}\right|^{2} \text { for } n=0,1, \ldots, N-1}$ \\
$\frac{\text { LINEAR MMSE ESTIMATION }}{\text { FOR } n=0 \text { TO } N-1 \text { DO }}$ \\
$\mathbf{f}_{n}=\left(\sigma^{2} \mathbf{I}_{W}+\mathbf{H}_{n} \mathbf{V}_{n} \mathbf{H}_{n}^{\mathrm{T}}\right)^{-1} \mathbf{h}_{n}$ \\
$s_{n}=\mathbf{f}_{n}^{\mathrm{T}} \mathbf{h}_{n}$ \\
$\lambda_{e x t}\left(c_{n}\right)=2\left(\mathbf{f}_{n}^{\mathrm{T}}\left(\mathbf{y}_{n}-\mathbf{H}_{n} \boldsymbol{\mu}_{n}\right)+\mu_{n} s_{n}\right) /\left(1-v_{n} s_{n}\right)$ \\
END \\
\hline OUTPUT \\
extrinsic LLRs $\lambda_{e x t}\left(c_{n}\right)$ for $n=0,1, \ldots, N-1$
\end{tabular}

$$
\begin{aligned}
\mathbf{f}_{n} & =\boldsymbol{\Sigma}_{n}^{-1} \mathbf{h}_{n}, \\
& =\mathbf{f}_{n}^{\mathrm{T}} /\left(1+\left(1-v_{n}\right) \mathbf{f}_{n}^{\mathrm{T}} \mathbf{h}_{n}\right) .
\end{aligned}
$$

The estimates $\hat{x}_{n}$ are now given by

$$
\begin{aligned}
\hat{x}_{n} & =\frac{\mathbf{f}_{n}^{\mathrm{T}}\left(\mathbf{y}_{n}-\mathbf{H}_{n} \boldsymbol{\mu}_{n}\right)+\mu_{n} s_{n}}{1+\left(1-v_{n}\right) s_{n}}, \\
s_{n} & =\mathbf{f}_{n}^{\mathrm{T}} \mathbf{h}_{n}=\mathbf{h}_{n}^{\mathrm{T}} \boldsymbol{\Sigma}_{n}^{-1} \mathbf{h}_{n} .
\end{aligned}
$$

To calculate the extrinsic LLRs $\lambda_{\text {ext }}\left(c_{n}\right)$, we have that the mean and the variance of the estimation error $e_{n}=\hat{x}_{n}-x_{n}$ are given by $\mathrm{E}\left\{e_{n}\right\}=0$ and under the constraint that $v_{n}$ is replaced by 1 yields

$$
\begin{aligned}
\operatorname{Var}\left\{e_{n}\right\} & =1-\mathbf{h}_{n}^{\mathrm{T}}\left(\boldsymbol{\Sigma}_{n}+\left(1-v_{n}\right) \mathbf{h}_{n} \mathbf{h}_{n}^{\mathrm{T}}\right)^{-1} \mathbf{h}_{n} \\
& =1-\mathbf{f}_{n}^{\mathrm{T}} \mathbf{h}_{n} /\left(1+\left(1-v_{n}\right) \mathbf{f}_{n}^{\mathrm{T}} \mathbf{h}_{n}\right) \\
& =1-s_{n} /\left(1+\left(1-v_{n}\right) s_{n}\right)
\end{aligned}
$$

where again Woodbury's identity was applied. Under the Gaussian assumption on the distribution of the estimation error $e_{n}$, the PDF $p\left(e_{n}\right)$ is given by $\mathcal{N}_{\mathbb{R}}\left(0,1-s_{n} /\left(1+\left(1-v_{n}\right) s_{n}\right)\right)$. Finally, from (13) the extrinsic LLRs $\lambda_{\text {ext }}\left(c_{n}\right)$ for BPSK modulation without precoding are given by

$$
\begin{aligned}
\lambda_{\text {ext }}\left(c_{n}\right) & =\ln \frac{\exp \left(-\left(\hat{x}_{n}-+1\right)^{2} /\left(2 \operatorname{Var}\left\{e_{n}\right\}\right)\right)}{\exp \left(-\left(\hat{x}_{n}--1\right)^{2} /\left(2 \operatorname{Var}\left\{e_{n}\right\}\right)\right)} \\
& =\frac{2 \hat{x}_{n}}{1-s_{n} /\left(1+\left(1-v_{n}\right) s_{n}\right)} \\
& =\frac{2\left(\mathbf{f}_{n}^{\mathrm{T}}\left(\mathbf{y}_{n}-\mathbf{H}_{n} \boldsymbol{\mu}_{n}\right)+\mu_{n} s_{n}\right)}{1-v_{n} s_{n}}, \\
\mathbf{f}_{n} & =\left(\sigma^{2} \mathbf{I}_{W}+\mathbf{H}_{n} \mathbf{V}_{n} \mathbf{H}_{n}^{\mathrm{T}}\right)^{-1} \mathbf{h}_{n}, \quad s_{n}=\mathbf{f}_{n}^{\mathrm{T}} \mathbf{h}_{n} .
\end{aligned}
$$

The complete SISO equalization algorithm using linear MMSE estimation is shown in Table III.

When the input LLRs $\lambda_{\text {ext }}^{\prime}\left(c_{n}\right)$ are 0 for all time steps $n$, e.g., for the initial equalization step in Fig. 5 or for usual linear MMSE estimation without incorporating prior knowledge about the symbols $x_{n}$, the means $\mu_{n}$ are equal to 0 and the variances $v_{n}$ are equal to 1 for all $n$. It follows that the coefficient vectors $\mathbf{f}_{n}$ are given by $\left(\sigma^{2} \mathbf{I}_{W}+\mathbf{H}_{n} \mathbf{H}_{n}^{\mathrm{T}}\right)^{-1} \mathbf{h}_{n}$ for all $n$, i.e., they are time-invariant and equal to the common linear MMSE equalizer [3]. The estimates $\hat{x}_{n}$ are in this case given by $\mathbf{f}_{n}^{\mathrm{T}} \mathbf{y}_{n}$ and the extrinsic LLRs $\lambda_{\text {ext }}\left(c_{n}\right)$ are given by $2 \mathbf{f}_{n}^{\mathrm{T}} \mathbf{y}_{n} /\left(1-s_{n}\right)$.

The BER performance of turbo equalization using linear MMSE estimation as equalization algorithm is shown in Fig. 8 for the same block length $K=510$ as in Fig. 6. Using the equalizer and the decoder once corresponds to separate equalization and decoding. Applying the steps outlined in Fig. 5 while computing the LLRs $\lambda_{\text {ext }}\left(c_{n}\right)$ according to (18) results in a performance gain even larger than that for turbo equalization based on symbol-based MAP detection shown in Fig. 6. After one iteration, $8 \mathrm{~dB}$ SNR $P / \sigma^{2}$ less are required to achieve a BER of $10^{-5}$. As in Fig. 6, the performance does not improve significantly using more iterations and, instead, approaches the BER performance of the soft decoder with an ISI-free channel (dotted line in Fig. 6). The BER performance of turbo equalization using the linear MMSE estimator is in general worse than that using the symbol-based MAP detector for turbo equalization for any SNR and any number of iterations, because the locally BEP-optimal MAP detector produces more reliable extrinsic LLRs $\lambda_{\text {ext }}\left(c_{n}\right)$. However, comparing the Figs. 6 and 8 reveals that the performance gap between the two diminishes with iterations of the turbo equalization algorithm and is negligible after 8 iterations.

The applicability of decision-feedback equalization for turbo equalization was investigated in [29]. However, the results were not promising as this amounts to replacing soft information $s^{\prime}\left(x_{n}\right)$ by a quantized value, which is inferior to the soft information itself. As such, the linear MMSE approach is, in fact, a decision feedback equalizer that employs soft decisions from the decoder, rather than hard decisions from the output of the equalizer or decoder.

The BER performance of turbo equalization is also affected by the Gaussian assumption made on the distribution of the estimation error

$$
e_{n}=\hat{x}_{n}-x_{n}=\frac{\mathbf{f}_{n}^{\mathrm{T}}\left(\mathbf{y}_{n}-\mathbf{H}_{n} \boldsymbol{\mu}_{n}\right)+\mu_{n} s_{n}}{1+\left(1-v_{n}\right) s_{n}}-x_{n} .
$$

Recall that this assumption allows computation of the extrinsic LLRs $\lambda_{\text {ext }}\left(c_{n}\right)$ efficiently. However, these LLRs are incorrect if 


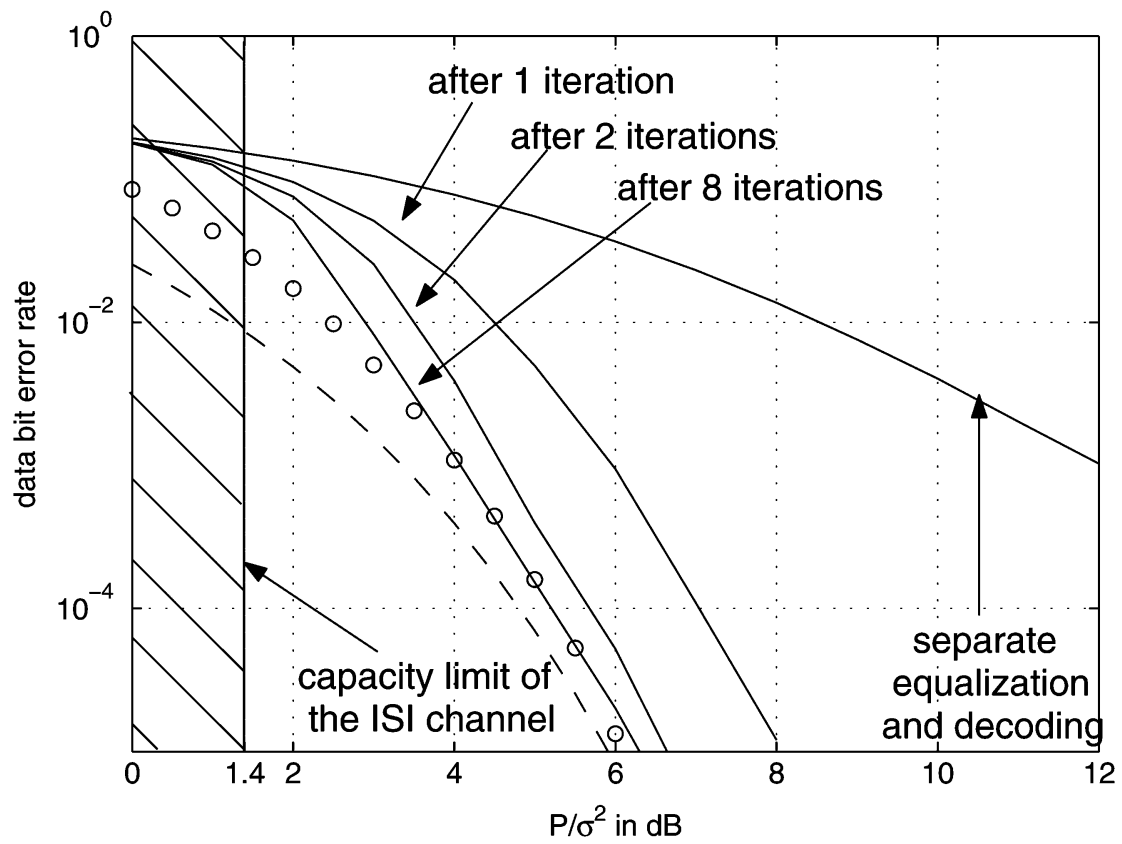

Fig. 8. Performance of turbo equalization for the communication system described in Section II-A using linear MMSE estimation. The BER performance (solid lines) is plotted for separate equalization and decoding as well as after 1,2, or 8 iterations. The dotted line corresponds to the BER performance of the FEC decoder when no ISI is introduced in the channel. The dashed line is a lower bound on the BER performance of any decoder. The blocklength considered is $K=510$ $(N=L=1024)$. An S-random interleaver with $\mathrm{S}=16$ is applied.

the true PDF $p\left(e_{n}\right)$ is not Gaussian. Using the exact PDF $p\left(e_{n}\right)$ for each time index $n$ to accurately calculate the equalizer output LLRs $\lambda_{\text {ext }}\left(c_{n}\right)$ is impractical. Fortunately, the Gaussian density $\mathcal{N}_{\mathbb{R}}\left(\mathrm{E}\left\{e_{n}\right\}, \operatorname{Var}\left\{e_{n}\right\}\right)$ given by $\mathcal{N}_{\mathbb{R}}\left(0,1-s_{n} /\left(1+\left(1-v_{n}\right) s_{n}\right)\right)$ is a good approximation to $p\left(e_{n}\right)$, although this observation does not hold for higher-order signal alphabets as shown in Section III.

Input LLRs $\lambda_{\text {ext }}^{\prime}\left(c_{n}\right)$ with large magnitude yield that $\mu_{n}=$ $x_{n}$ and $v_{n}=0$ hold. The coefficient vector $\mathbf{f}_{n}=\boldsymbol{\Sigma}_{n}^{-1} \mathbf{h}_{n}$ and the term $s_{n}=\mathbf{f}_{n}^{\mathrm{T}} \mathbf{h}_{n}$ are in this case given by

$$
\mathbf{f}_{n}=\left(\sigma^{2} \mathbf{I}_{W}\right)^{-1} \mathbf{h}_{n}=\mathbf{h}_{n} / \sigma^{2} \text { and } s_{n}=\mathbf{f}_{n}^{\mathrm{T}} \mathbf{h}_{n}=P_{h} / \sigma^{2},
$$

where $P_{h}=\sum_{i=0}^{M_{h}}\left|h_{i}\right|^{2}$ is the power of the ISI channel. The estimates $\hat{x}_{n}$ are given by (20), at the bottom of the page. The estimation error $e_{n}$ is given by

$$
e_{n}=\frac{\mathbf{h}_{n}^{\mathrm{T}} \mathbf{w}_{n}+P_{h} \cdot x_{n}}{\sigma^{2}+P_{h}}-x_{n}=\frac{\mathbf{h}_{n}^{\mathrm{T}} \mathbf{w}_{n}-\sigma^{2} \cdot x_{n}}{\sigma^{2}+P_{h}} .
$$

The PDF $p\left(e_{n}\right)$ is the superposition of the two conditional PDFs $p\left(e_{n} \mid x_{n}=x\right)$, which are given by $\mathcal{N}_{\mathbb{R}}\left(-x \sigma^{2} /\left(\sigma^{2}+\right.\right.$ $\left.\left.P_{h}\right), P_{h} \sigma^{2} /\left(\sigma^{2}+P_{h}\right)^{2}\right)$, weighted with $s^{\prime}\left(x_{n}=x\right)=0.5$

$$
\begin{aligned}
p\left(e_{n}\right)=\kappa \cdot \exp \left(-\left|e_{n}\right|^{2} /\left(2 P_{h} \sigma^{2} /\left(\sigma^{2}+P_{h}\right)^{2}\right)\right) & \cdot \cosh \left(e_{n} /\left(2 P_{h}\right)\right) .
\end{aligned}
$$

The constant $\kappa$ assures that $p\left(e_{n}\right)$ is a PDF. This distribution closely matches the Gaussian approximation $\mathcal{N}_{\mathbb{R}}\left(0,1-s_{n} /(1+\right.$ $\left.\left.\left(1-v_{n}\right) s_{n}\right)\right)$ given by $\mathcal{N}_{\mathbb{R}}\left(0, \sigma^{2} /\left(\sigma^{2}+P_{h}\right)^{2}\right.$.

The performance results in Figs. 6 and 8 indicate that the linear MMSE estimator is a viable alternative to the symbolbased MAP detector, since the BER performance is nearly identical after a few iterations. However, this result should be interpreted with care, since the required number of iterations and the required blocklength $K$ may be beyond the specifications of a given application. For separate equalization and decoding and within early iterations, there is still a considerable performance gap between the two approaches.

$$
\begin{aligned}
\hat{x}_{n} & =\frac{\mathbf{h}_{n}^{\mathrm{T}}\left(\mathbf{y}_{n}-\mathbf{H}_{n} \boldsymbol{\mu}_{n}\right) / \sigma^{2}+\mu_{n} \cdot P_{h} / \sigma^{2}}{1+P_{h} / \sigma^{2}} \\
& =\frac{\mathbf{h}_{n}^{\mathrm{T}}\left(\mathbf{y}_{n}-\mathbf{H}_{n}\left(\mu_{n-M_{h}-W_{2}} \ldots \mu_{n-1} 0 \mu_{n+1} \ldots \mu_{n+W_{1}}\right)^{\mathrm{T}}\right)}{\sigma^{2}+P_{h}} .
\end{aligned}
$$




\section{COMmunication SySTEMS USING PSK OR QAM MODULATION}

\section{A. System Model}

In this section, the transmitted symbols $x_{n}$ are now chosen from a $2^{q}$-ary QAM signal alphabet $\mathcal{S}$, i.e., they are in general complex-valued. The $q$-tuples are directly mapped to a symbol $x_{n}$ using a Gray mapping function.

The ISI channel model is identical to (1) except that the channel coefficients $h_{i}$ are complex-valued and the noise samples $w_{n}$ are circularly symmetric complex Gaussian distributed with variance $\sigma^{2}$

$$
\begin{gathered}
y_{n}=w_{n}+\sum_{i=0}^{M_{h}} h_{i} x_{n-i}, \\
n=0,1, \ldots, L-1, \quad \text { or } \quad \mathbf{y}=\mathbf{H x}+\mathbf{w} .
\end{gathered}
$$

The PDF $p\left(w_{n}\right)$ is therefore given by $\mathcal{N}_{\mathbb{C}}\left(0, \sigma^{2}\right)$, where $\mathcal{N}_{\mathbb{C}}\left(\mu, \sigma^{2}\right)$ denotes

$$
p(x)=\exp \left(-|x-\mu|^{2} / \sigma^{2}\right) /\left(\pi \sigma^{2}\right) .
$$

The same length-3 unit power example channel as in Section II-A is used to illustrate the BER performance of the algorithms described here. The system matrix $\mathbf{H}$ is constructed as in (2).

The same memory- $\left(M_{c}=2\right)$ convolutional code as in Section II-A is applied such that the system code rate $R$ is given by

$$
R=\log _{2}|\mathcal{S}| \cdot R_{\mathrm{fec}}=q \cdot R_{\mathrm{fec}}=q \cdot \frac{1}{2+2 / K} .
$$

The signal alphabets $\mathcal{S}$ can be chosen to satisfy the power constraint $\mathrm{E}\left\{\left|x_{n}\right|^{2}\right\}=P=1$ under the IUD assumption on the symbols $x_{n}$. BER performance results are obtained using an 8-PSK alphabet. This choice is made for simplicity, i.e., the following derivations apply to any memory- $M_{h}$ ISI channel with complex-valued coefficients $h_{i}$ and any finite signal alphabet $\mathcal{S}$.

\section{B. SISO Equalization Based on Symbol-Based MAP Detection}

The focus of this section is on the second step in Fig. 5, which is to compute the extrinsic LLR $\lambda_{\text {ext }}\left(c_{n}\right)$ from the observations $\mathbf{y}$ and all input LLRs $\lambda_{\text {ext }}^{\prime}\left(c_{0}\right), \ldots, \lambda_{\text {ext }}^{\prime}\left(c_{N-1}\right)$ except $\lambda_{\text {ext }}^{\prime}\left(c_{n}\right)$.
We first consider MAP detection as in Table I. The trellis describing a memory- $M_{h}$ ISI channel with input symbols $x_{n}$ from a signal alphabet $\mathcal{S}$ of size $2^{q}$ has $2^{q M_{h}}$ states. The entries $\gamma_{n}(i, j)=p\left(y_{n} \mid v_{n}=v_{i, j}\right) \cdot s^{\prime}\left(x_{n}=x_{i, j}\right)$ of the $2^{q M_{h}} \times 2^{q M_{h}}$ matrices $\Gamma_{n}$ are given by the first equation at the bottom of the page, for $i, j=0,1, \ldots, 2^{q M_{h}}-1$, where $v_{i, j}$ and $x_{i, j}$ follow from the ISI channel trellis and $\kappa=1 /\left(\pi \sigma^{2}\right)$. The scaling $\kappa$ can be neglected if a normalization step is added in the forward and backward BCJR recursions.

Each symbol $x_{n}$ depends on exactly $q$ code bits $\mathbf{c}_{n}=$ $\left(c_{q n} \ldots c_{q n+q-1}\right)$, such that a symbol probability $s^{\prime}\left(x_{n}\right)$ is the product of $q$ extrinsic code bit probabilities, i.e., $s^{\prime}\left(x_{n}=x\right)=$ $\prod_{k=0}^{q-1} s_{\text {ext }}^{\prime}\left(c_{q n+k}=m_{k}\right)$ with $x=\operatorname{map}\left(m_{0}, \ldots, m_{q-1}\right)$. For example, the probability $s^{\prime}\left(x_{n}=-1\right)$ for an 8-PSK alphabet might be given by $s_{\text {ext }}^{\prime}\left(c_{3 n}=1\right) s_{\text {ext }}^{\prime}\left(c_{3 n+1}=0\right) s_{\text {ext }}^{\prime}\left(c_{3 n+2}=\right.$ $0)$. The soft mapping can be rewritten in terms of extrinsic LLRs $\lambda_{\text {ext }}^{\prime}\left(c_{n}\right)$

$$
\begin{aligned}
s^{\prime}\left(x_{n}=x\right) & =\prod_{k=0}^{q-1} \frac{\exp \left(-m_{k} \cdot \lambda_{\text {ext }}^{\prime}\left(c_{q n+k}\right)\right)}{1+\exp \left(-\lambda_{\text {ext }}^{\prime}\left(c_{q n+k}\right)\right)}, \\
x & =\operatorname{map}\left(m_{0}, \ldots, m_{q-1}\right) .
\end{aligned}
$$

Practical implementations of the BCJR algorithm most often store the quantity $\ln \boldsymbol{\Gamma}_{n}$, whose entries are easily computed from the LLRs $\lambda_{\text {ext }}^{\prime}\left(c_{n}\right)$, as shown in the second equation at the bottom of the page, where $x_{i, j}=\operatorname{map}\left(m_{0}, \ldots, m_{q-1}\right)$ and all unnecessary normalization factors are omitted.

The soft demapping from the posterior probabilities $P\left(x_{n} \mid \mathbf{y}\right)$ to the extrinsic LLRs $\lambda_{\text {ext }}\left(c_{q n+k}\right)$ is performed by marginalizing $c_{q n+k}$ in the corresponding joint probability $P\left(\mathbf{c}_{n} \mid \mathbf{y}\right)$, as shown in the first equation at the bottom of the next page, with $\mathbf{m}=\left(m_{0}, m_{1}, \ldots, m_{q-1}\right)$, where $\mathbf{U}(u)$ follows from the trellis describing the ISI channel and $\mathbf{f}_{n}$ and $\mathbf{b}_{n}$ are part of the BCJR algorithm. To ensure that $\lambda_{\text {ext }}\left(c_{q n+k}\right)$ does not depend on $\lambda_{\text {ext }}^{\prime}\left(c_{q n+k}\right)$, we observe that computing $\lambda_{\text {ext }}\left(c_{q n+k}\right)$ with the extrinsic transition matrix, as shown in the second equation at the bottom of the next page, ensures that $\lambda_{\text {ext }}\left(c_{q n+k}\right)$ does not depend on $\lambda_{\text {ext }}^{\prime}\left(c_{q n+k}\right)$, only. This relationship follows from (22) and that the product $\prod_{j=0}^{q-1} \exp \left(-m_{j} \cdot \lambda_{\text {ext }}^{\prime}\left(c_{q n+j}\right)\right)$ can be factored out of the expression $\mathbf{b}_{n+1}^{\mathrm{T}}\left(\mathbf{U}(\operatorname{map}(\mathbf{m})) \odot \boldsymbol{\Gamma}_{n}\right) \mathbf{f}_{n}$.

The system rate $R$ given an 8-PSK signal alphabet equals $R=q \cdot R_{\mathrm{fec}}=1.5$ bits per channel use for large block lengths $L$. A minimum SNR $P / \sigma^{2}$ of $4.7 \mathrm{~dB}$ is required in order that the

$$
\boldsymbol{\Gamma}_{n}[j, i]= \begin{cases}\kappa \cdot \exp \left(-\frac{\left|y_{n}-v_{i, j}\right|^{2}}{\sigma^{2}}\right) s^{\prime}\left(x_{n}=x_{i, j}\right), & (i j) \text { corresponds to a valid trellis branch } \\ 0, & \text { otherwise }\end{cases}
$$

$$
\ln \Gamma_{n}[j, i]= \begin{cases}-\frac{\left|y_{n}-v_{i, j}\right|^{2}}{\sigma^{2}}-\sum_{k=0}^{q-1} m_{k} \cdot \lambda_{\text {ext }}^{\prime}\left(c_{q n+k}\right), & (i j) \text { corresponds to a valid trellis branch } \\ -\infty, & \text { otherwise }\end{cases}
$$




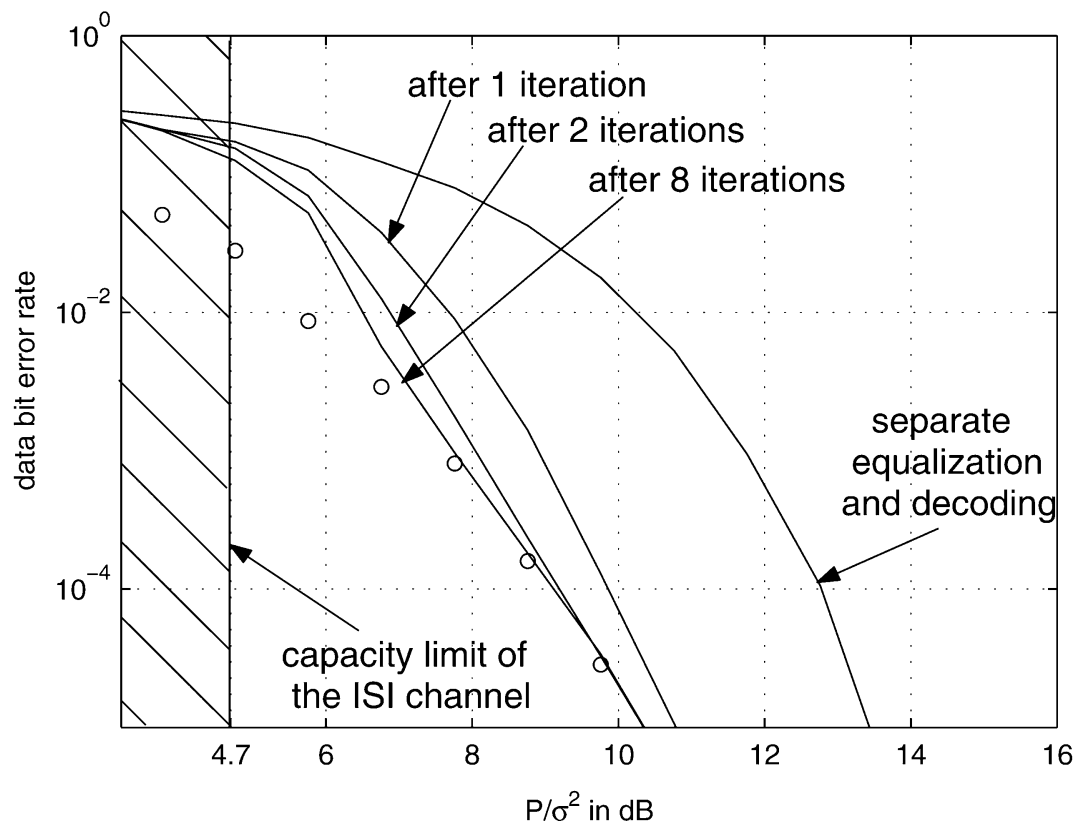

Fig. 9. Performance of turbo equalization for the communication system described in Section III-A using symbol-based MAP detection. The BER performance (solid lines) is plotted for separate equalization and decoding as well as after 1,2, or 8 iterations. The dotted line corresponds to the BER performance of bitinterleaved coded modulation given an ISI-free channel after 8 demapping-decoding iterations. The block length considered is $K=510(N=1024$ and $L=$ 342). An S-random interleaver with $\mathrm{S}=16$ is applied.

constrained IUD capacity $C_{c, \text { iud }}(\mathcal{S})$ for 8 -PSK is larger than $R=1.5$. Thus, the capacity limit for arbitrarily reliable data transmission using the given system configuration is $4.7 \mathrm{~dB}$ SNR.

The BER performance of turbo equalization using the symbol-based MAP detector as equalization algorithm is shown in Fig. 9 for the same block length $K=510$ as in the previously shown simulations in Figs. 6, and 8. Applying the steps outlined in Fig. 5 results in a performance improvement in the first iteration, since $1.5 \mathrm{~dB} \mathrm{SNR} P / \sigma^{2}$ is gained at a BER of $10^{-5}$. As in Section II-D, more iterations do not improve the performance significantly and, instead, the BER performance of the soft decoder (10) without ISI (dotted line in Fig. 9) is a lower bound. In this case, the channel model (21) simplifies to $y_{n}=x_{n}+w_{n}$ such that the extrinsic LLRs $\lambda_{\text {ext }}\left(c_{n}\right)$ are given by (23), at the bottom of the next page, where the PDF $p\left(y_{n} \mid x_{n}\right)$ is given by $\mathcal{N}_{\mathbb{C}}\left(x_{n}, \sigma^{2}\right)$.

The equalization step given an ISI-free channel is merely a soft demapping operation. A communication system consisting of a FEC code, an interleaver, and a mapper, which transmits data over an (ISI-free) AWGN channel is called bit-interleaved coded modulation (BICM) in the literature [89]. The explana-

$$
\begin{aligned}
\lambda_{\text {ext }}\left(c_{q n+k}\right) & =\ln \frac{s_{\text {ext }}\left(c_{q n+k}=0\right)}{s_{\text {ext }}\left(c_{q n+k}=1\right)} \\
& =\left.\ln \frac{\sum_{\mathbf{m} \in \mathbb{F}_{2}^{q}: m_{k}=0} P\left(x_{n}=\operatorname{map}(\mathbf{m}) \mid \mathbf{y}\right)}{\sum_{\mathbf{m} \in \mathbb{F}_{2}^{q}: m_{k}=1} P\left(x_{n}=\operatorname{map}(\mathbf{m}) \mid \mathbf{y}\right)}\right|_{\text {without using } \lambda_{\text {ext }}^{\prime}\left(c_{q n+k}\right)} \\
& =\left.\ln \frac{\sum_{\mathbf{m} \in \mathbb{F}_{2}^{q}: m_{k}=0} \mathbf{b}_{n+1}^{\mathrm{T}}\left(\mathbf{U}(\operatorname{map}(\mathbf{m})) \odot \boldsymbol{\Gamma}_{n}\right) \mathbf{f}_{n}}{\sum_{\mathbf{m} \in \mathbb{F}_{2}^{q}: m_{k}=1} \mathbf{b}_{n+1}^{\mathrm{T}}\left(\mathbf{U}(\operatorname{map}(\mathbf{m})) \odot \boldsymbol{\Gamma}_{n}\right) \mathbf{f}_{n}}\right|_{\text {without using } \lambda_{\text {ext }}^{\prime}\left(c_{q n+k}\right)}
\end{aligned}
$$

and

$$
\boldsymbol{\Gamma}_{\text {ext }, n}[j, i]= \begin{cases}\exp \left(-\left|y_{n}-v_{i, j}\right|^{2} / \sigma^{2}\right), & (i j) \text { corresponds to a valid trellis branch } \\ 0, & \text { otherwise, }\end{cases}
$$

$$
\lambda_{\text {ext }}\left(c_{q n+k}\right)=\ln \frac{\sum_{\mathbf{m} \in \mathbb{F}_{2}^{q}: m_{k}=0} \mathbf{b}_{n+1}^{\mathrm{T}}\left(\mathbf{U}(\operatorname{map}(\mathbf{m})) \odot \boldsymbol{\Gamma}_{\text {ext }, n}\right) \mathbf{f}_{n} \prod_{j=0: j \neq k}^{q-1} \exp \left(-m_{j} \cdot \lambda_{\text {ext }}^{\prime}\left(c_{q n+j}\right)\right)}{\sum_{\mathbf{m} \in \mathbb{F}_{2}^{q}: m_{k}=1} \mathbf{b}_{n+1}^{\mathrm{T}}\left(\mathbf{U}(\operatorname{map}(\mathbf{m})) \odot \boldsymbol{\Gamma}_{\text {ext }, n}\right) \mathbf{f}_{n} \prod_{j=0: j \neq k}^{q-1} \exp \left(-m_{j} \cdot \lambda_{\text {ext }}^{\prime}\left(c_{q n+j}\right)\right)},
$$


tions at the end of Section II-D and the results in [89] show that the BER performance of BICM is the same as that of the BEP-optimal decoder (6) for ideal interleaving. Since the performance of turbo equalization closely approaches that of BICM as shown in Fig. 9, we again observe that turbo equalization achieves the performance of the BEP-optimal decoder for the communication system. However, the BER performance of BICM is far away from the capacity limit, which calls for additional system design, e.g., including precoding or the use of another mapping function $\operatorname{map}(\cdot)[90]$.

\section{SISO Equalization Based on MMSE Estimation}

It is also natural to extend the SISO equalizer based on linear MMSE equalization derived in Section II-E to higher-order signal alphabets. As in (14), only a length- $W$ window $\mathbf{y}_{n}$ of observations $y_{n}$ is considered to compute the MMSE-optimal estimate $\hat{x}_{n}$ of $x_{n}$ minimizing the MSE $\mathrm{E}\left\{\left|\hat{x}_{n}-x_{n}\right|^{2}\right\}$. The MMSE-optimal estimate $\hat{x}_{n}$ of the complex-valued symbol $x_{n}$ is not a linear, but a widely linear [91] combination of the complex-valued symbols $y_{n}$

$$
\hat{x}_{n}=\mathrm{E}\left\{x_{n}\right\}+\mathbf{u}_{n}\left(\mathbf{y}_{n}-\mathrm{E}\left\{\mathbf{y}_{n}\right\}\right)+\mathbf{v}_{n}\left(\mathbf{y}_{n}-\mathrm{E}\left\{\mathbf{y}_{n}\right\}\right)^{*},
$$

where

$$
\begin{aligned}
\mathbf{u}_{n}= & \left(\operatorname{Cov}\left\{x_{n}, \mathbf{y}_{n}\right\}-\operatorname{Cov}\left\{x_{n}, \mathbf{y}_{n}^{*}\right\}\right. \\
& \left.\times\left(\operatorname{Cov}\left\{\mathbf{y}_{n}, \mathbf{y}_{n}\right\}^{-1}\right)^{*} \operatorname{Cov}\left\{\mathbf{y}_{n}, \mathbf{y}_{n}^{*}\right\}^{*}\right) \mathbf{R}_{n}, \\
\mathbf{v}_{n}= & \left(\operatorname{Cov}\left\{x_{n}, \mathbf{y}_{n}^{*}\right\}-\operatorname{Cov}\left\{x_{n}, \mathbf{y}_{n}\right\}\right. \\
& \left.\times \operatorname{Cov}\left\{\mathbf{y}_{n}, \mathbf{y}_{n}\right\}^{-1} \operatorname{Cov}\left\{\mathbf{y}_{n}, \mathbf{y}_{n}^{*}\right\}\right) \mathbf{R}_{n}^{*}, \\
\mathbf{R}_{n}= & \left(\operatorname{Cov}\left\{\mathbf{y}_{n}, \mathbf{y}_{n}\right\}-\operatorname{Cov}\left\{\mathbf{y}_{n}, \mathbf{y}_{n}^{*}\right\}\right. \\
& \left.\times\left(\operatorname{Cov}\left\{\mathbf{y}_{n}, \mathbf{y}_{n}\right\}^{-1}\right)^{*} \operatorname{Cov}\left\{\mathbf{y}_{n}, \mathbf{y}_{n}^{*}\right\}^{*}\right)^{-1} .
\end{aligned}
$$

It is also possible to consider the real-valued estimate $\left(\hat{x}_{n, \mathrm{R}} \hat{x}_{n, \mathrm{I}}\right)$ of $\left(\operatorname{Re}\left\{x_{n}\right\} \operatorname{Im}\left\{x_{n}\right\}\right)$, which linearly depends on the $2 W$ real-valued observations $\operatorname{Re}\left\{\mathbf{y}_{n}\right\}$ and $\operatorname{Im}\left\{\mathbf{y}_{n}\right\}$, as in [92]. However, it turns out in Section IV that low-complexity approximate implementations of (24) are easier to derive based on the description in $\mathbb{C}$.

The statistics $\mu_{n}=\mathrm{E}\left\{x_{n}\right\}$ and $v_{n}=\operatorname{Var}\left\{x_{n}\right\}$ of the transmitted symbols $x_{n}$ given an arbitrary signal alphabet $\mathcal{S}$ are given by

$$
\begin{aligned}
& \mu_{n}=\sum_{x \in \mathcal{S}} x \cdot s^{\prime}\left(x_{n}=x\right) \quad \text { and } \\
& v_{n}=\sum_{x \in \mathcal{S}}\left|x-\mu_{n}\right|^{2} \cdot s^{\prime}\left(x_{n}=x\right) .
\end{aligned}
$$

Recall that the soft information $s^{\prime}\left(x_{n}\right)$ is a symbol probability that $x_{n}$ takes on a value from $\mathcal{S}$. The pseudocovariances
$\operatorname{Cov}\left\{x_{n}, \mathbf{y}_{n}^{*}\right\}$ and $\operatorname{Cov}\left\{\mathbf{y}_{n}, \mathbf{y}_{n}^{*}\right\}$ using the ISI channel model (21) are

$$
\begin{aligned}
\operatorname{Cov}\left\{x_{n}, \mathbf{y}_{n}^{*}\right\} & =\operatorname{Cov}\left\{x_{n}, x_{n}^{*}\right\} \mathbf{h}_{n}^{\mathrm{T}} \quad \text { and } \\
\operatorname{Cov}\left\{\mathbf{y}_{n}, \mathbf{y}_{n}^{*}\right\} & =\mathbf{H}_{n} \operatorname{Cov}\left\{\mathbf{x}_{n}, \mathbf{x}_{n}^{*}\right\} \mathbf{H}_{n}^{\mathrm{T}}=\mathbf{H}_{n} \mathbf{\Psi}_{n} \mathbf{H}_{n}^{\mathrm{T}} .
\end{aligned}
$$

From the independence assumption on the symbols $x_{n}, \Psi_{n}=$ $\operatorname{Cov}\left\{\mathbf{x}_{n}, \mathbf{x}_{n}^{*}\right\}$ is a diagonal matrix with the pseudovariances

$$
\psi_{n}=\operatorname{Cov}\left\{x_{n}, x_{n}^{*}\right\}=\sum_{x \in \mathcal{S}}\left(x-\mu_{n}\right)^{2} \cdot s^{\prime}\left(x_{n}=x\right)
$$

on the main diagonal. Combining all results, the estimates $\hat{x}_{n}$ are given by

$$
\hat{x}_{n}=\mu_{n}+\mathbf{u}_{n}\left(\mathbf{y}_{n}-\mathbf{H}_{n} \boldsymbol{\mu}_{n}\right)+\mathbf{v}_{n}\left(\mathbf{y}_{n}-\mathbf{H}_{n} \boldsymbol{\mu}_{n}\right)^{*},
$$

where $\mathbf{u}_{n}=\left(v_{n} \mathbf{h}_{n}^{\mathrm{H}}-\psi_{n} \mathbf{h}_{n}^{\mathrm{T}}\left(\boldsymbol{\Sigma}_{n}^{-1}\right)^{*}\left(\mathbf{H}_{n} \mathbf{\Psi}_{n} \mathbf{H}_{n}^{\mathrm{T}}\right)^{*}\right) \mathbf{R}_{n}$, $\mathbf{v}_{n}=\left(\psi_{n} \mathbf{h}_{n}^{\mathrm{T}}-v_{n} \mathbf{h}_{n}^{\mathrm{H}} \boldsymbol{\Sigma}_{n}^{-1} \mathbf{H}_{n} \boldsymbol{\Psi}_{n} \mathbf{H}_{n}^{\mathrm{T}}\right) \mathbf{R}_{n}^{*}$, and $\mathbf{R}_{n}=$ $\left(\boldsymbol{\Sigma}_{n}-\mathbf{H}_{n} \boldsymbol{\Psi}_{n} \mathbf{H}_{n}^{\mathrm{T}}\left(\boldsymbol{\Sigma}_{n}^{-1}\right)^{*}\left(\mathbf{H}_{n} \boldsymbol{\Psi}_{n} \mathbf{H}_{n}^{\mathrm{T}}\right)^{*}\right)^{-1}$.

A special case are IUD symbols $x_{n}$, i.e., $s\left(x_{n}\right)$ is a uniform PMF, which holds in the initial equalization step of turbo equalization or for separate equalization and decoding. In this case, $\mu_{n}=0$ and $v_{n}=1$ holds for all $n$ given many signal alphabets. Moreover, the pseudovariances $\psi_{n}$ vanish for all $n$, i.e., the symbols $x_{n}$ are circularly symmetric except for the BPSK alphabet. The corresponding constraint on the signal alphabet is $\sum_{x \in \mathcal{S}} x^{2}=0$. The coefficient vector $\mathbf{v}$ vanishes if $\psi_{n}=0$ holds for all $n$ such that the estimation problem simplifies greatly to

$$
\hat{x}_{n}=\mathbf{h}_{n}^{\mathrm{H}}\left(\sigma^{2} \mathbf{I}_{W}+\mathbf{H}_{n} \mathbf{H}_{n}^{\mathrm{H}}\right)^{-1} \mathbf{y}_{n} .
$$

This solution is similar to that in (8) for MMSE estimation of real-valued parameters.

Given a $2^{q}$-ary signal alphabet $\mathcal{S}, q$ extrinsic LLRs $\lambda_{\text {ext }}\left(c_{n}\right)$ must be produced per estimate $\hat{x}_{n}$. To this end, all equalizer input LLRs except $\lambda_{\text {ext }}^{\prime}\left(c_{n}\right)$ can be used. Using the standard assumption that the estimation error $e_{n}=\hat{x}_{n}-x_{n}$ is Gaussian distributed, the estimate $\hat{x}_{n}$ can be written as $\hat{x}_{n}=e_{n}+x_{n}$, where the distribution $p\left(e_{n}\right)$ is in general a complex Gaussian PDF with mean $\mu=\mathrm{E}\left\{e_{n}\right\}$, variance $\sigma^{2}=\operatorname{Var}\left\{e_{n}\right\}$, and pseudovariance $\psi=\operatorname{Cov}\left\{e_{n}, e_{n}^{*}\right\}$ :

$$
\begin{aligned}
p\left(e_{n}\right)= & \exp \left(-\frac{\left|e_{n}-\mu\right|^{2}}{\sigma^{2}-|\psi|^{2} / \sigma^{2}}\right. \\
& \left.+\operatorname{Re}\left\{\frac{\left(e_{n}^{*}-\mu^{*}\right)^{2} \cdot \psi / \sigma^{2}}{\sigma^{2}-|\psi|^{2} / \sigma^{2}}\right\}\right) / \sqrt{\pi^{2} \cdot\left(\sigma^{4}-|\psi|^{2}\right)} .
\end{aligned}
$$

Such a PDF is briefly denoted $\mathcal{N}_{\mathbb{C}}\left(\mu, \sigma^{2}, \psi\right)$ in the sequel. The relationship $\hat{x}_{n}=e_{n}+x_{n}$ is similar to the ISI channel model

$$
\begin{aligned}
\lambda_{\text {ext }}\left(c_{q n+k}\right) & =\left.\ln \frac{\sum_{\mathbf{m} \in \mathbb{F}_{2}^{q}: m_{k}=0} P\left(x_{n}=\operatorname{map}(\mathbf{m}) \mid y_{n}\right)}{\sum_{\mathbf{m} \in \mathbb{F}_{2}^{q}: m_{k}=1} P\left(x_{n}=\operatorname{map}(\mathbf{m}) \mid y_{n}\right)}\right|_{\text {without using } \lambda_{\text {ext }}^{\prime}\left(c_{q n+k}\right)} \\
& =\ln \frac{\sum_{\mathbf{m} \in \mathbb{F}_{2}^{q}: m_{k}=0} p\left(y_{n} \mid x_{n}=\operatorname{map}(\mathbf{m})\right) \prod_{j=0: j \neq k}^{q-1} \exp \left(-m_{j} \cdot \lambda_{\text {ext }}^{\prime}\left(c_{q n+j}\right)\right)}{\sum_{\mathbf{m} \in \mathbb{F}_{2}^{q}: m_{k}=1} p\left(y_{n} \mid x_{n}=\operatorname{map}(\mathbf{m})\right) \prod_{j=0: j \neq k}^{q-1} \exp \left(-m_{j} \cdot \lambda_{\text {ext }}^{\prime}\left(c_{q n+j}\right)\right)},
\end{aligned}
$$


TABLE IV

$O\left(L \cdot W^{2}\right)$ SiSO EQUALIZATION ALGORITHM BASED ON LINEAR MMSE ESTIMATION OF BPSK-MODULATED SyMBOLS

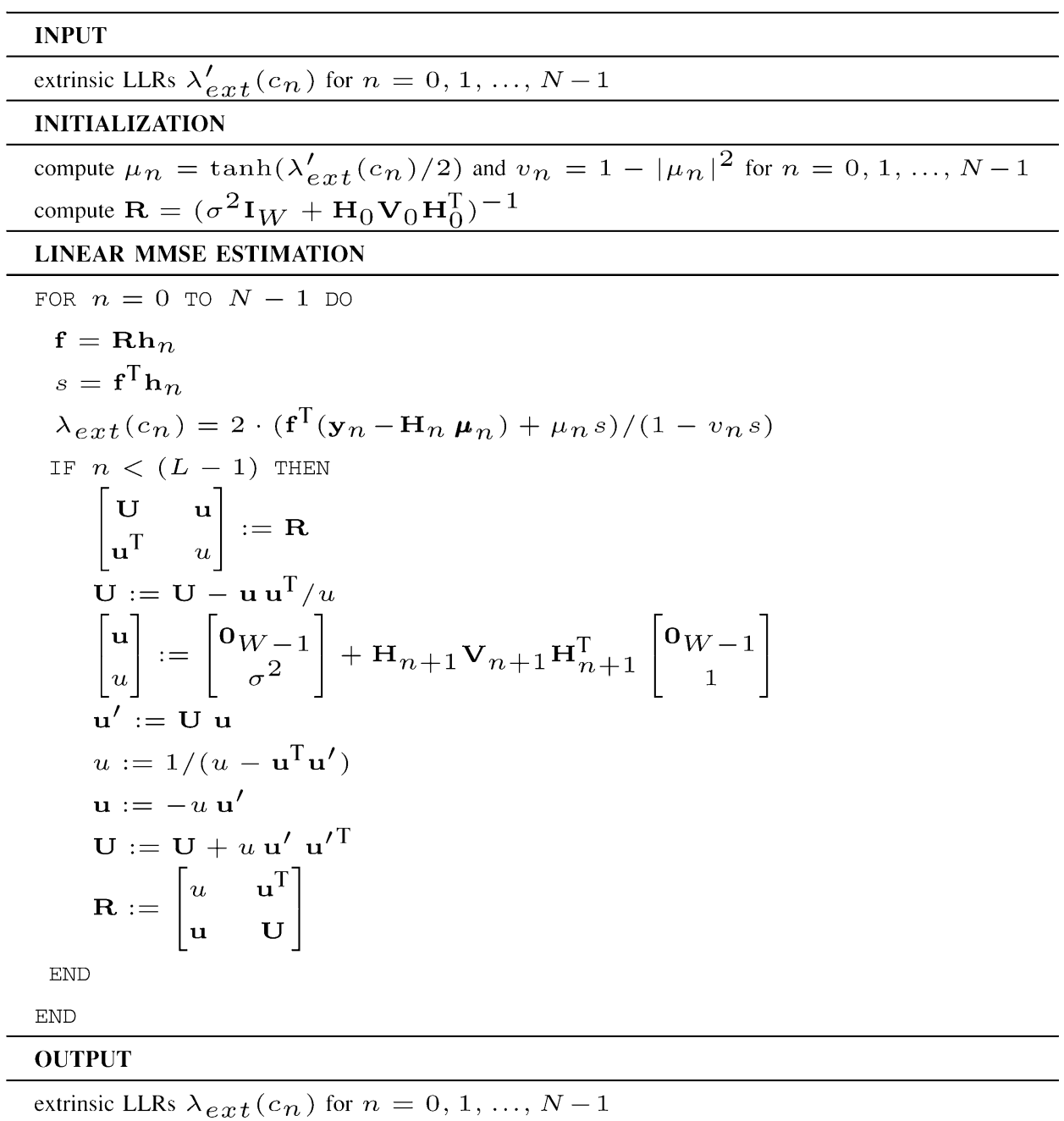

(21) in the ISI-free case, i.e., $y_{n}=x_{n}+w_{n}$, where $w_{n}$ is distributed with $\mathcal{N}_{\mathbb{C}}\left(0, \sigma^{2}\right)$. It follows that the method (23) for generating the extrinsic output LLRs $\lambda_{\text {ext }}\left(c_{n}\right)$ of a symbol-based MAP detector in the ISI-free case can be applied here as well, as shown in the equation at the bottom of the page, where $p\left(\hat{x}_{n} \mid x_{n}\right)$ is given by $\mathcal{N}_{\mathbb{C}}\left(x_{n}+\mathrm{E}\left\{e_{n}\right\}, \operatorname{Var}\left\{e_{n}\right\}, \operatorname{Cov}\left\{e_{n}, e_{n}^{*}\right\}\right)$ and $\mathbf{m}=\left(m_{0}, m_{1}, \ldots, m_{q-1}\right)$. An advantage of using $P\left(x_{n} \mid \hat{x}_{n}\right)$ is the complexity used to compute $\hat{x}_{n}$, which is polynomial in the window length $W$ as shown in Section IV, compared to the exponential order $O\left(2^{q M_{h}}\right)$ for computing $P\left(x_{n} \mid \mathbf{y}\right)$.
The estimate $\hat{x}_{n}$ must not depend on $\lambda_{\text {ext }}^{\prime}\left(c_{q n+k}\right)$ while computing $\lambda_{\text {ext }}\left(c_{q n+k}\right)$ to assure that $\lambda_{\text {ext }}\left(c_{q n+k}\right)$ is a valid extrinsic LLR. Since $\hat{x}_{n}$ depends on the $q$ LLRs $\lambda_{\text {ext }}^{\prime}\left(c_{q n+k}\right)$, $k=0, \ldots, q-1$, via $\mu_{n}, v_{n}$, and $\psi_{n}$, it seems that $\hat{x}_{n}$ needs to be recomputed $q$ times for each $\lambda_{\text {ext }}\left(c_{q n+k}\right), k=0, \ldots, q-1$, with different statistics $\mu_{n}, v_{n}$, and $\psi_{n}$ obtained by setting the corresponding input LLR $\lambda_{\text {ext }}^{\prime}\left(c_{q n+k}\right)$ to 0 . To avoid this costly recalculation, only one estimate $\hat{x}_{n}$ for each $\lambda_{\text {ext }}\left(c_{q n+k}\right), k=$ $0, \ldots, q-1$, is computed while imposing the IUD assumption on the particular symbol $x_{n}$. It follows that $\mu_{n}, v_{n}$, and $\psi_{n}$ should

$$
\begin{aligned}
\lambda_{\text {ext }}\left(c_{q n+k}\right) & =\left.\ln \frac{\sum_{\mathbf{m} \in \mathbb{F}_{2}^{q}: m_{k}=0} P\left(x_{n}=\operatorname{map}(\mathbf{m}) \mid \hat{x}_{n}\right)}{\sum_{\mathbf{m} \in \mathbb{F}_{2}^{q}: m_{k}=1} P\left(x_{n}=\operatorname{map}(\mathbf{m}) \mid \hat{x}_{n}\right)}\right|_{\text {without using } \lambda_{\text {ext }}^{\prime}\left(c_{q n+k}\right)} \\
& =\ln \frac{\sum_{\mathbf{m} \in \mathbb{F}_{2}^{q}: m_{k}=0} p\left(\hat{x}_{n} \mid x_{n}=\operatorname{map}(\mathbf{m})\right) \prod_{j=0: j \neq k}^{q-1} \exp \left(-m_{j} \cdot \lambda_{\text {ext }}^{\prime}\left(c_{q n+j}\right)\right)}{\sum_{\mathbf{m} \in \mathbb{F}_{2}^{q}: m_{k}=1} p\left(\hat{x}_{n} \mid x_{n}=\operatorname{map}(\mathbf{m})\right) \prod_{j=0: j \neq k}^{q-1} \exp \left(-m_{j} \cdot \lambda_{\text {ext }}^{\prime}\left(c_{q n+j}\right)\right)},
\end{aligned}
$$


TABLE V

SISO EQUALIZATION ALGORITHM BASED ON WIDELY LINEAR MMSE ESTIMATION

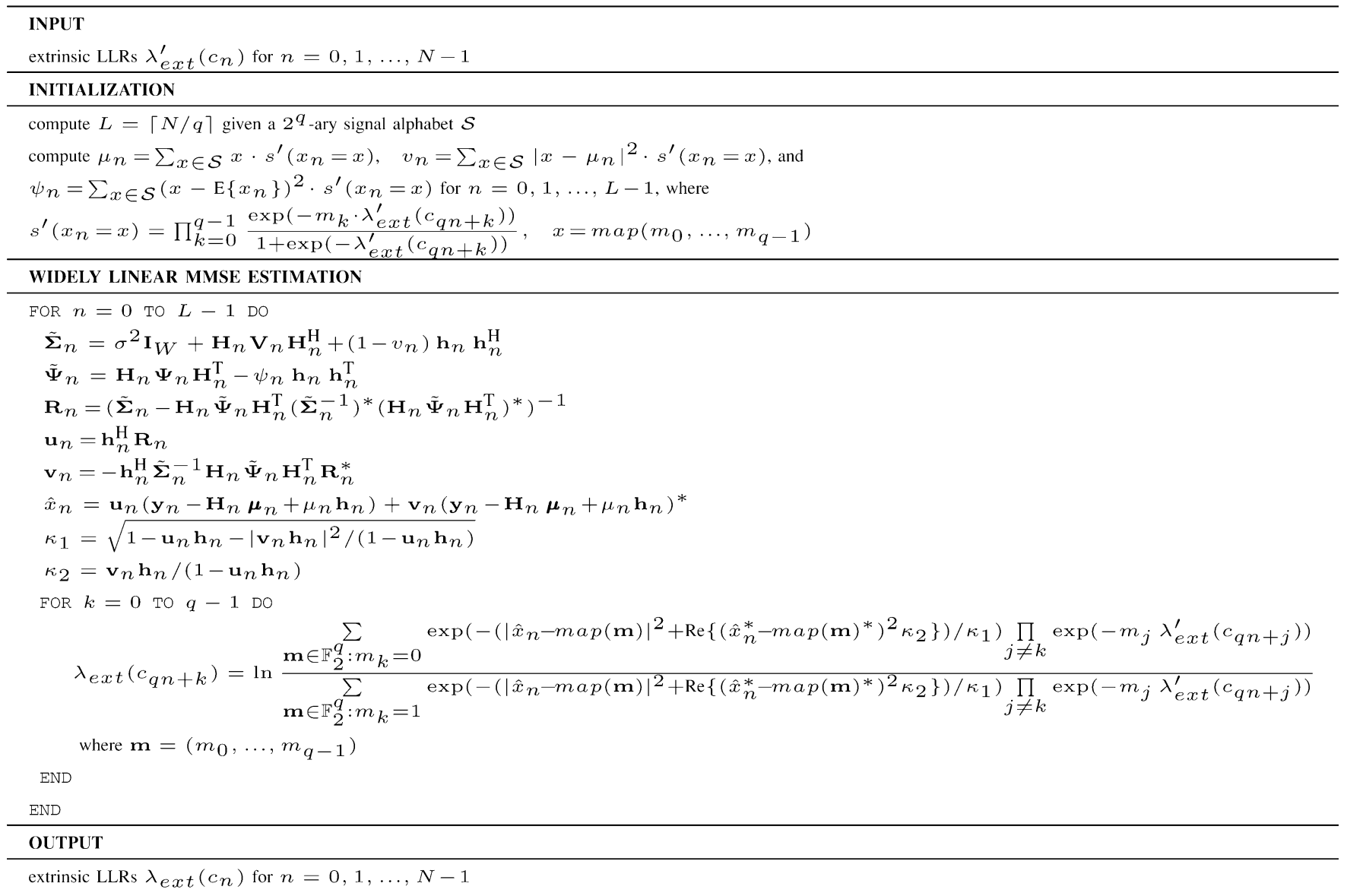

be replaced by 0,1 , and 0 , respectively, while computing $\hat{x}_{n}$, which changes (27) to

$$
\begin{aligned}
\hat{x}_{n}=\mathbf{u}_{n}\left(\mathbf{y}_{n}-\mathbf{H}_{n} \boldsymbol{\mu}_{n}+\right. & \left.\mu_{n} \mathbf{h}_{n}\right) \\
& +\mathbf{v}_{n}\left(\mathbf{y}_{n}-\mathbf{H}_{n} \boldsymbol{\mu}_{n}+\mu_{n} \mathbf{h}_{n}\right)^{*},
\end{aligned}
$$

where $\mathbf{u}_{n}=\mathbf{h}_{n}^{\mathrm{H}} \mathbf{R}_{n}, \mathbf{v}_{n}=-\mathbf{h}_{n}^{\mathrm{H}} \tilde{\boldsymbol{\Sigma}}_{n}^{-1} \mathbf{H}_{n} \tilde{\mathbf{\Psi}}_{n} \mathbf{H}_{n}^{\mathrm{T}} \mathbf{R}_{n}^{*}$, and $\mathbf{R}_{n}=$ $\left(\tilde{\boldsymbol{\Sigma}}_{n}-\mathbf{H}_{n} \tilde{\boldsymbol{\Psi}}_{n} \mathbf{H}_{n}^{\mathrm{T}}\left(\tilde{\boldsymbol{\Sigma}}_{n}^{-1}\right)^{*}\left(\mathbf{H}_{n} \tilde{\boldsymbol{\Psi}}_{n} \mathbf{H}_{n}^{\mathrm{T}}\right)^{*}\right)^{-1}$ using the updated matrices $\tilde{\boldsymbol{\Sigma}}_{n}=\boldsymbol{\Sigma}_{n}+\left(1-v_{n}\right) \mathbf{h}_{n} \mathbf{h}_{n}^{\mathrm{H}}$ and $\tilde{\boldsymbol{\Psi}}_{n}=\boldsymbol{\Psi}_{n}-$ $\psi_{n} \mathbf{h}_{n} \mathbf{h}_{n}^{\mathrm{T}}$.
The statistics $\mathrm{E}\left\{e_{n}\right\}, \operatorname{Var}\left\{e_{n}\right\}$, and $\operatorname{Cov}\left\{e_{n}, e_{n}^{*}\right\}$ of the estimation error $e_{n}$ under the constraints that $v_{n}$ and $\psi_{n}$ be replaced by 1 and 0 , respectively, yield $\mathrm{E}\left\{e_{n}\right\}=0$, and

$$
\operatorname{Var}\left\{e_{n}\right\}=1-\mathbf{u}_{n} \mathbf{h}_{n} \text { and } \operatorname{Cov}\left\{e_{n}, e_{n}^{*}\right\}=\mathbf{v}_{n} \mathbf{h}_{n} .
$$

The PDF $p\left(e_{n}\right)$ is, thus, given by $\mathcal{N}_{\mathbb{C}}\left(0,1-\mathbf{u}_{n} \mathbf{h}_{n}, \mathbf{v}_{n} \mathbf{h}_{n}\right)$ under the Gaussian assumption, i.e., (30), as shown at the bottom of the page. The extrinsic LLRs $\lambda_{\text {ext }}\left(c_{n}\right)$ are given by (31), at the bottom of the page, where $\kappa_{1}=\sqrt{1-\mathbf{u}_{n} \mathbf{h}_{n}-\left|\mathbf{v}_{n} \mathbf{h}_{n}\right|^{2} /\left(1-\mathbf{u}_{n} \mathbf{h}_{n}\right)}, \kappa_{2}=\mathbf{v}_{n} \mathbf{h}_{n} /(1-$

$$
p\left(e_{n}\right)=\exp \left(-\frac{\left|e_{n}\right|^{2}+\operatorname{Re}\left\{\left(e_{n}^{*}\right)^{2} \operatorname{Cov}\left\{e_{n}, e_{n}^{*}\right\} / \operatorname{Var}\left\{e_{n}\right\}\right\}}{\operatorname{Var}\left\{e_{n}\right\}-\left|\operatorname{Cov}\left\{e_{n}, e_{n}^{*}\right\}\right|^{2} / \operatorname{Var}\left\{e_{n}\right\}}\right) /\left(\pi \sqrt{\operatorname{Var}\left\{e_{n}\right\}^{2}-\left|\operatorname{Cov}\left\{e_{n}, e_{n}^{*}\right\}\right|^{2}}\right)
$$

$$
\lambda_{\text {ext }}\left(c_{q n+k}\right)=\ln \frac{\sum_{\mathbf{m} \in \mathbb{F}_{2}^{q}: m_{k}=0} \exp \left(-\frac{\left|\hat{x}_{n}-\operatorname{map}(\mathbf{m})\right|^{2}+\operatorname{Re}\left\{\left(\hat{x}_{n}^{*}-\operatorname{map}(\mathbf{m})^{*}\right)^{2} \kappa_{2}\right\}}{\kappa_{1}}\right) \prod_{j \neq k} \exp \left(-m_{j} \lambda_{\text {ext }}^{\prime}\left(c_{q n+j}\right)\right)}{\sum_{\mathbf{m} \in \mathbb{F}_{2}^{q}: m_{k}=1} \exp \left(-\frac{\left|\hat{x}_{n}-\operatorname{map}(\mathbf{m})\right|^{2}+\operatorname{Re}\left\{\left(\hat{x}_{n}^{*}-\operatorname{map}(\mathbf{m})^{*}\right)^{2} \kappa_{2}\right\}}{\kappa_{1}}\right) \prod_{j \neq k} \exp \left(-m_{j} \lambda_{\text {ext }}^{\prime}\left(c_{q n+j}\right)\right)},
$$


$\left.\mathbf{u}_{n} \mathbf{h}_{n}\right)$, and $\mathbf{m}=\left(m_{0}, \ldots, m_{q-1}\right)$. The complete SISO equalization algorithm based on widely linear MMSE estimation is shown in Table V.

When the input LLRs $\lambda_{\text {ext }}^{\prime}\left(c_{n}\right)$ are 0 for all $n$, e.g., for the initial equalization step in Fig. 5 or for usual MMSE estimation without incorporating prior knowledge about the symbols $x_{n}$, all means $\mu_{n}$ and pseudovariances $\psi_{n}$ are 0 and all variances $v_{n}$ are 1 . As shown in (28), the estimates $\hat{x}_{n}$ are given by $\mathbf{u}_{n} \mathbf{y}_{n}$ with $\mathbf{u}_{n}=\mathbf{h}_{n}^{\mathrm{H}}\left(\sigma^{2} \mathbf{I}_{W}+\mathbf{H}_{n} \mathbf{H}_{n}^{\mathrm{H}}\right)^{-1}$ under this condition. The statistics of the estimation error $e_{n}$ simplify to $\mathrm{E}\left\{e_{n}\right\}=$ $0, \operatorname{Var}\left\{e_{n}\right\}=1-\mathbf{u}_{n} \mathbf{h}_{n}$, and $\operatorname{Cov}\left\{e_{n}, e_{n}^{*}\right\}=0$, i.e., the estimation error is circularly symmetric and distributed with $\mathcal{N}_{\mathbb{C}}\left(0, \operatorname{Var}\left\{e_{n}\right\}\right)$ under the Gaussian assumption, which simplifies the computation of the extrinsic LLRs $\lambda_{\text {ext }}\left(c_{n}\right)$ to that shown in the equation at the bottom of the page, with $\mathbf{m}=$ $\left(m_{0}, \ldots, m_{q-1}\right)$.

The BER performance of turbo equalization using the widely linear MMSE estimator is exhibited in Fig. 10 for the same block length $K=510$ as in Fig. 9. The system model and 8PSK signal alphabet are as in Section III-A and the window parameters $W=11, W_{1}=6$, and $W_{2}=4$ are similar to the choice in Section II-E.

It turns out that the Gaussian assumption made on the estimation error $e_{n}=\hat{x}_{n}-x_{n}$, which is, in particular for higher-order signal alphabets, not accurate [93], is a limiting factor in this case. The inconsistency apparent in the derivation for the linear MMSE estimator used for BPSK modulation in Section II-E does not cause a significant performance degradation as shown in Fig. 8, due to the good match between $p\left(e_{n}\right)$ and the Gaussian approximation. In contrast, the degradation is significant in Fig. 10. A remedy to this problem could be a more accurate modeling of $p\left(e_{n}\right)$ as proposed in [93] at the expense that the calculation of the extrinsic LLRs $\lambda_{\text {ext }}\left(c_{n}\right)$ becomes more complex.

\section{IMPLEMENTATION ISSUES}

The focus of this section is on the computational complexity of the MMSE estimation algorithm. The computational complexity is measured per SISO equalization task, which is to produce $N$ extrinsic LLRs $\lambda_{\text {ext }}\left(c_{n}\right)$ from $N$ input LLRs $\lambda_{\text {ext }}^{\prime}\left(c_{n}\right)$ and $L$ observations $y_{n}$. This task has to be repeated for each iteration of the turbo equalization algorithm. The complexity depends on the following system parameters: the size $|\mathcal{S}|$ of the signal alphabet $\mathcal{S}$, the memory $M_{h}$ of the ISI channel, the estimator window length $W$, and the number $L$ of symbols $x_{n}$ transmitted over the channel.

\section{A. Trellis-Based Approaches}

SISO equalization based on symbol-based MAP detection can be implemented on a trellis with $|\mathcal{S}|^{M_{h}}$ states as shown in Table I (for BPSK modulation) and in Section III-A for arbitrary signal alphabets. It follows that the computational complexity of a SISO equalization task is of order $O\left(L \cdot|\mathcal{S}|^{M_{h}}\right)$. The same holds for sequence-based MAP detection using the Viterbi algorithm [6], [83]. The exponential increase of the required number of operations in the channel memory $M_{h}$, in particular for signal alphabets of large size $|\mathcal{S}|$, is a serious problem in a practical implementation. As a remedy, numerous approximations of both symbol- and sequence-based MAP detection exists, which aim to use an alternative trellis describing the ISI channel with fewer states [44].

\section{B. Linear MMSE Estimation of BPSK-Modulated Symbols}

SISO equalization of BPSK-modulated symbols $x_{n}$ based on linear MMSE estimation is described in Table III. The complexity order of the SISO equalization task, which is to calculate the $N=L$ output LLRs $\lambda_{\text {ext }}\left(c_{n}\right)$, is governed by the inversion of the time-varying $W \times W$ matrix $\boldsymbol{\Sigma}_{n}=\sigma^{2} \mathbf{I}_{W}+\mathbf{H}_{n} \mathbf{V}_{n} \mathbf{H}_{n}^{\mathrm{T}}$ to calculate the coefficient vector $\mathbf{f}_{n}=\boldsymbol{\Sigma}_{n}^{-1} \mathbf{h}_{n}$ for each time step $n$. This operation is of order $O\left(W^{3}\right)$ such that the complexity of the entire SISO equalization task is of order $O\left(L \cdot W^{3}\right)$. However, the structured time-dependence of the matrix $\boldsymbol{\Sigma}_{n}$ admits a fast recursive solution for computing $\mathbf{f}_{n}$ that has complexity that is $O\left(W^{2}\right)$. The time-recursive update algorithm introduced in [30] uses the following partitioning scheme:

$$
\boldsymbol{\Sigma}_{n}=\left[\begin{array}{ll}
s_{\text {old }} & \mathbf{s}_{\text {old }}^{\mathrm{T}} \\
\mathbf{S}_{\text {old }} & \mathbf{S}_{\text {old }}
\end{array}\right] \quad \text { and } \quad \boldsymbol{\Sigma}_{n+1}=\left[\begin{array}{ll}
\mathbf{S}_{\text {new }} & \mathbf{S}_{\text {new }} \\
\mathbf{s}_{\text {new }}^{\mathrm{T}} & s_{\text {new }}
\end{array}\right] \text {, }
$$

where the $\mathbf{S}_{\text {old }}, \mathbf{S}_{\text {new }}$ are $(W-1) \times(W-1)$ matrices, $\mathbf{S}_{\text {old }}, \mathbf{S}_{\text {new }}$ are length $(W-1)$ column vectors, and $s_{\text {old }}, s_{\text {new }}$ are scalars. The subscript old denotes quantities at time step $n$ and new denotes quantities at time step $n+1$. A similar partitioning scheme is introduced for the inverses of $\boldsymbol{\Sigma}_{n}$ and $\boldsymbol{\Sigma}_{n+1}$

$$
\begin{gathered}
\boldsymbol{\Sigma}_{n}^{-1}=\mathbf{U}_{n}=\left[\begin{array}{ll}
u_{\text {old }} & \mathbf{u}_{\text {old }}^{\mathrm{T}} \\
\mathbf{u}_{\text {old }} & \mathbf{U}_{\text {old }}
\end{array}\right] \text { and } \\
\boldsymbol{\Sigma}_{n+1}^{-1}=\mathbf{U}_{n+1}=\left[\begin{array}{ll}
\mathbf{U}_{\text {new }} & \mathbf{u}_{\text {new }} \\
\mathbf{u}_{\text {new }}^{\mathrm{T}} & u_{\text {new }}
\end{array}\right] .
\end{gathered}
$$

$$
\lambda_{\operatorname{ext}}\left(c_{q n+k}\right)=\ln \frac{\sum_{\mathbf{m} \in \mathbb{F}_{2}^{q}: m_{k}=0} \exp \left(-\frac{\left|\mathbf{u}_{n} \mathbf{y}_{n}-\operatorname{map}(\mathbf{m})\right|^{2}}{1-\mathbf{u}_{n} \mathbf{h}_{n}}\right) \prod_{j \neq k} \exp \left(-m_{j} \lambda_{\text {ext }}^{\prime}\left(c_{q n+j}\right)\right)}{\sum_{\mathbf{m} \in \mathbb{F}_{2}^{q}: m_{k}=1} \exp \left(-\frac{\left|\mathbf{u}_{n} \mathbf{y}_{n}-\operatorname{map}(\mathbf{m})\right|^{2}}{1-\mathbf{u}_{n} \mathbf{h}_{n}}\right) \prod_{j \neq k} \exp \left(-m_{j} \lambda_{\text {ext }}^{\prime}\left(c_{q n+j}\right)\right)},
$$




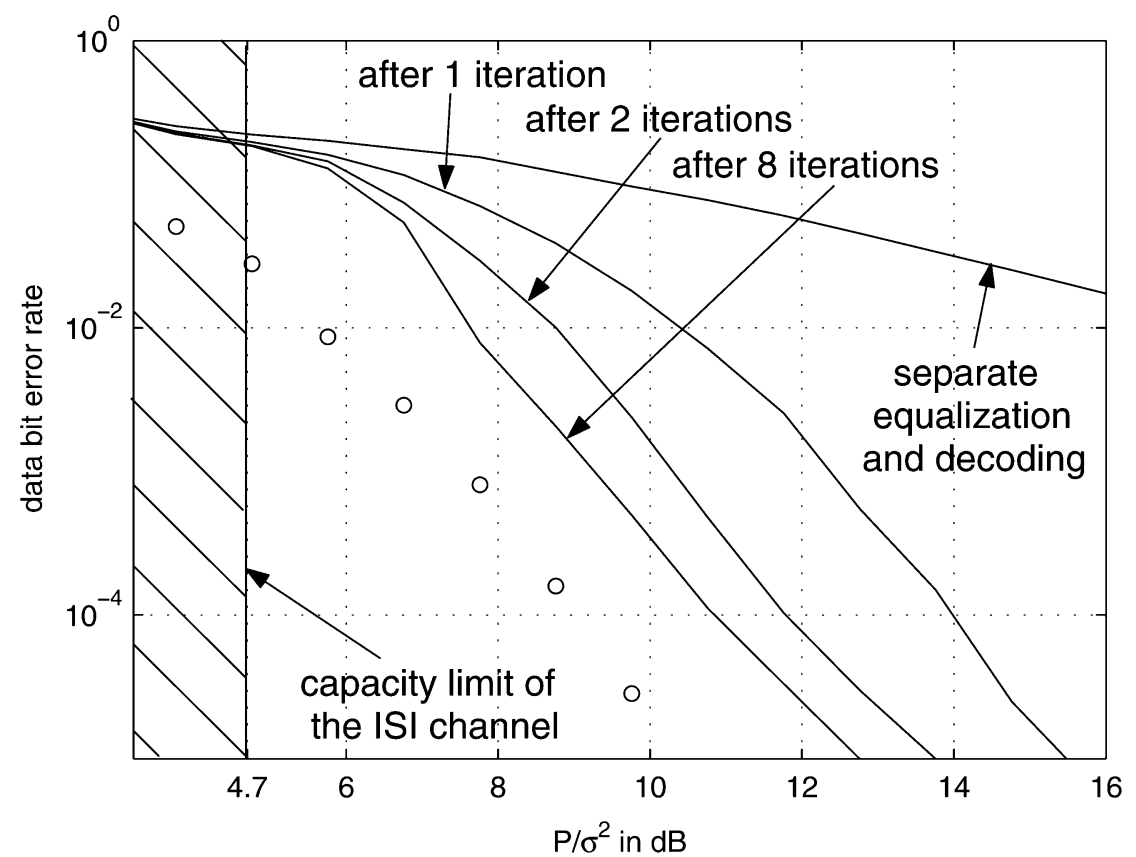

Fig. 10. Performance of turbo equalization for the communication system described in Section III-A using widely linear MMSE estimation. The BER performance (solid lines) is plotted for separate equalization and decoding as well as after 1,2, or 8 iterations. The dotted line corresponds to the BER performance of bitinterleaved coded modulation given an ISI-free channel after 8 demapping-decoding iterations. The considered block length is $K=510$ ( $N=1024$ and $L=$ 342). An S-random interleaver with $\mathrm{S}=16$ is applied.

The efficient recursive implementation of the equalization algorithm in Table III arises from noting that the submatrices $\mathbf{S}_{\text {old }}$ and $\mathbf{S}_{\text {new }}$ are identical

$$
\begin{aligned}
\mathbf{S}_{\text {old }} & =\mathbf{S}_{\text {new }} \\
& =\sigma^{2} \mathbf{I}_{W-1}+\mathbf{H}_{n}^{\prime} \operatorname{Diag}\left\{v_{n-M_{h}-W_{2}+1} \cdots v_{n+W_{1}}\right\} \mathbf{H}_{n}^{\prime \mathrm{T}},
\end{aligned}
$$

where $\mathbf{H}_{n}^{\prime}$ is the $(W-1) \times\left(W+M_{h}-1\right)$ submatrix $\mathbf{H}_{n}[1$ : $\left.W-1,1: W+M_{h}-1\right]$ of $\mathbf{H}_{n}$, i.e., $\mathbf{H}_{n}^{\prime}$ is the submatrix $\mathbf{H}\left[n-W_{2}+1: n+W_{1}, n-W_{2}-M_{h}+1: n+W_{1}\right]$ of the system matrix $\mathbf{H}$. The proposed recursive algorithm computes first $\mathbf{S}_{\text {old }}^{-1}$ from $\mathbf{U}_{n}$, sets $\mathbf{S}_{\text {old }}^{-1}=\mathbf{S}_{\text {new }}^{-1}$, and computes $\mathbf{U}_{n+1}$ from $\mathbf{S}_{\text {new }}^{-1}$.

The inverse $\mathbf{S}_{\text {old }}^{-1}$ of the submatrix $\mathbf{S}_{\text {old }}$ of $\boldsymbol{\Sigma}_{n}$ is expressed in terms of components of $\mathbf{U}_{n}$ by solving $\boldsymbol{\Sigma}_{n} \mathbf{U}_{n}=\mathbf{I}_{W}$ using (32) and (33)

$$
\begin{aligned}
\mathbf{S}_{\text {old }} \mathbf{U}_{\text {old }}+\mathbf{s}_{\text {old }} \mathbf{u}_{\text {old }}^{\mathrm{T}} & =\mathbf{I}_{W-1}, \\
\mathbf{S}_{\text {old }} \mathbf{u}_{\text {old }}+\mathbf{s}_{\text {old }} u_{\text {old }} & =\mathbf{0}_{W-1}, \\
\rightarrow \mathbf{S}_{\text {old }}^{-1} & =\mathbf{U}_{\text {old }}-\mathbf{u}_{\text {old }} \mathbf{u}_{\text {old }}^{\mathrm{T}} / u_{\text {old }} .
\end{aligned}
$$

By solving $\boldsymbol{\Sigma}_{n+1} \mathbf{U}_{n+1}=\mathbf{I}_{W}$, the quantities $\mathbf{U}_{\text {new }}, \mathbf{u}_{\text {new }}$, and $u_{\text {new }}$ are expressed in terms of $\mathbf{S}_{\text {new }}, \mathbf{s}_{\text {new }}$, and $s_{\text {new }}$

$$
\begin{aligned}
\mathbf{s}_{\text {new }}^{\prime} & =\mathbf{S}_{\text {new }}^{-1} \mathbf{s}_{\text {new }}, \\
u_{\text {new }} & =1 /\left(s_{\text {new }}-\mathbf{s}_{\text {new }}^{\mathrm{T}} \mathbf{s}_{\text {new }}^{\prime}\right), \\
\mathbf{u}_{\text {new }} & =-u_{\text {new }} \mathbf{s}_{\text {new }}^{\prime}, \\
\mathbf{U}_{\text {new }} & =\mathbf{S}_{\text {new }}^{-1}+u_{\text {new }} \mathbf{s}_{\text {new }}^{\prime} \mathbf{s}_{\text {new }}^{\prime}{ }^{\mathrm{T}},
\end{aligned}
$$

where the equations are ordered to optimize the computation by using the intermediate vector $\mathbf{s}_{\text {new }}^{\prime}$. The matrix $\mathbf{S}_{\text {new }}^{-1}$ is equal to
$\mathbf{S}_{\text {old }}^{-1}$ and the quantities $\mathbf{S}_{\text {new }}$ and $s_{\text {new }}$ are computed using the definition of $\boldsymbol{\Sigma}_{n}$ and its partitioning in (32):

$$
\left[\begin{array}{c}
\mathbf{s}_{\text {new }} \\
s_{\text {new }}
\end{array}\right]=\left[\begin{array}{c}
\mathbf{0}_{W-1} \\
\sigma^{2}
\end{array}\right]+\mathbf{H}_{n+1} \mathbf{V}_{n+1} \mathbf{H}_{n+1}^{\mathrm{T}}\left[\begin{array}{c}
\mathbf{0}_{W-1} \\
1
\end{array}\right]
$$

Assembling $\mathbf{U}_{n+1}$ from $\mathbf{U}_{\text {new }}, \mathbf{u}_{\text {new }}$, and $u_{\text {new }}$ completes the recursive algorithm.

To bootstrap the time-recursive update algorithm, an initialization of $\mathbf{U}_{n}$ at the starting time step $n=0$ is required, e.g., by computing $\mathbf{U}_{0}=\left(\sigma^{2} \mathbf{I}_{W}+\mathbf{H}_{0} \mathbf{V}_{0} \mathbf{H}_{0}^{\mathrm{T}}\right)^{-1}$. This operation can be trivial when the block of transmitted symbols $x_{n}$ starts with a preamble of at least $W+M_{h}$ symbols known to the receiver, which yields $\mathbf{V}_{0}=\mathbf{0}_{W+M_{h}}$ and, thus, $\mathbf{U}_{0}=\mathbf{I}_{W} / \sigma^{2}$.

The complete SISO equalization based on linear MMSE estimation of BPSK-modulated symbols is summarized in Table IV. The most demanding operations to compute the output LLRs $\lambda_{\text {ext }}\left(c_{n}\right)$ are matrix-vector multiplications such as $\mathbf{S}_{\text {new }}^{-1} \mathbf{S}_{\text {new }}$ and outer vector products such as $\mathbf{s}_{\text {new }}^{\prime} \mathbf{s}_{\text {new }}^{\prime}{ }^{\mathrm{T}}$, which are both of order $O\left(W^{2}\right)$. The complexity of the complete SISO equalization algorithm is therefore $O\left(L \cdot W^{2}\right)$.

\section{Approximate Linear MMSE Estimation of BPSK-Modulated Symbols}

A further complexity reduction is possible by using a time invariant coefficient vector $\mathbf{f}$ to compute the estimates $\hat{x}_{n}$, i.e., the time varying coefficient vector $\mathbf{f}_{n}$ in (16) is replaced by the length- $W$ vector $\mathbf{f}$ that remains constant for all $n=0,1, \ldots, L-1$,

$$
\hat{x}_{n}=\kappa \cdot\left(\mathbf{f}^{\mathrm{T}}\left(\mathbf{y}_{n}-\mathbf{H}_{n} \boldsymbol{\mu}_{n}\right)+\mu_{n} \mathbf{f}^{\mathrm{T}} \mathbf{h}_{n}\right),
$$




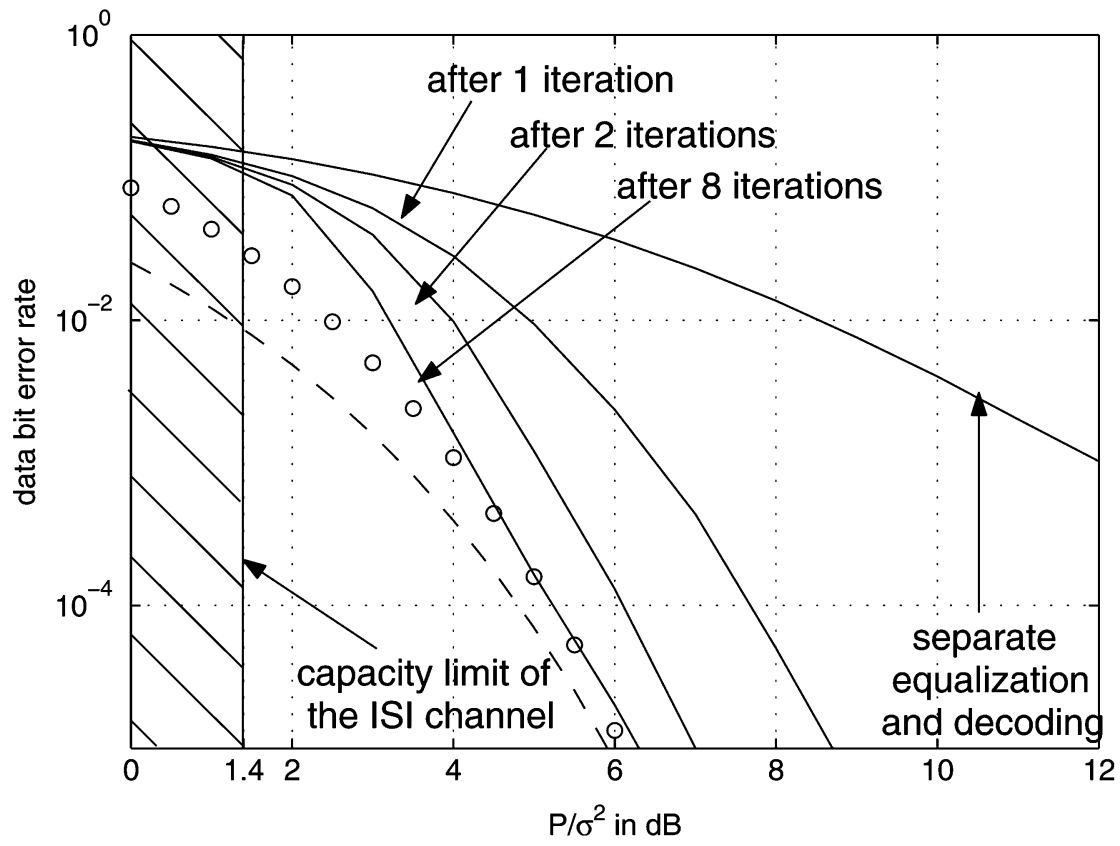

Fig. 11. Performance of turbo equalization for the communication system described in Section II-A using approximate linear MMSE estimation of BPSK-modulated symbols. The BER performance (solid lines) is plotted for separate equalization and decoding as well as after 1, 2, or 8 iterations. The dotted line corresponds to the BER performance of the FEC decoder when no ISI is introduced in the channel. The dashed line is a lower bound on the BER performance of any decoder. The blocklength considered is $K=510(N=L=1024)$. An S-random interleaver with $\mathrm{S}=16$ is applied.

where $\kappa$ is a constant. Recall that $\kappa$ is equal to $1 /(1+(1-$ $\left.\left.v_{n}\right) \mathbf{f}_{n}^{\mathrm{T}} \mathbf{h}_{n}\right)$ in (16), where it assures that $v_{n}$ is replaced by 1 while computing $\hat{x}_{n}$ as shown in Section II-E. The complexity order of computing the estimates $\hat{x}_{n}$ via (36) is $O(W)$, and the results in Fig. 11 show that this approach has an excellent complexity/ performance trade-off. Clearly, using (36) makes sense only if the ISI channel impulse response is time invariant, i.e., $\mathbf{H}_{n}$ must be constant over time.

Of crucial interest is the actual choice for $\mathbf{f}$. In [29] was proposed to set $\mathbf{f}$ equal to the MMSE-optimal solution in case all input LLRs $\lambda_{\text {ext }}^{\prime}\left(c_{n}\right)$ are 0 , e.g., for the initial equalization step. The coefficient vector $\mathbf{f}$ is given by $\left(\sigma^{2} \mathbf{I}_{W}+\mathbf{H}_{n} \mathbf{H}_{n}^{\mathrm{T}}\right)^{-1} \mathbf{h}_{n}$ under this condition. While this choice yields a good BER performance of turbo equalization for the first few iterations, the performance degrades significantly compared to the MMSE-optimal approach in Table III. The reason for this behavior is explained in [29].

Another method also proposed in [29] is to set $\mathbf{f}$ equal to the MMSE-optimal solution in case all input LLRs $\lambda_{\text {ext }}^{\prime}\left(c_{n}\right)$ have a large magnitude such that $v_{n}=0$ holds for all $n$, e.g., after convergence of turbo equalization. The coefficient vector $\mathbf{f}$ is given by $\mathbf{h}_{n} / \sigma^{2}$ under this condition, which is the matched filter to the ISI channel impulse response already derived in (20). Performing turbo equalization based on this equalization algorithm has a severe convergence problem, i.e., large SNR $P / \sigma^{2}$ is required to achieve performance improvements over the iterations [29]. This is not surprising, since the input LLRs $\lambda_{\text {ext }}^{\prime}\left(c_{n}\right)$ are initially 0 , a condition, for which the choice $\mathbf{f}=\mathbf{h}_{n} / \sigma^{2}$ is certainly not suited.

A possible remedy to cure the weaknesses of both proposals is to start with $\mathbf{f}=\left(\sigma^{2} \mathbf{I}_{W}+\mathbf{H}_{n} \mathbf{H}_{n}^{\mathrm{T}}\right)^{-1} \mathbf{h}_{n}$ for the first few iterations, where the input LLRs $\lambda_{\text {ext }}^{\prime}\left(c_{n}\right)$ are usually of small magnitude. Subject to a quality criterion evaluating the output
LLRs $\lambda_{\text {ext }}\left(c_{n}\right)$, the SISO equalizer switches to $\mathbf{f}=\mathbf{h}_{n} / \sigma^{2}$, which is suitable for input LLRs with large magnitude. This hybrid equalization approach is explained in detail in [29].

A more powerful solution for $\mathbf{f}$ was derived in [30], which includes the above two choices as special cases

$$
\mathbf{f}=\left(\sigma^{2} \mathbf{I}_{W}+\mathbf{H}_{n} \overline{\mathbf{V}} \mathbf{H}_{n}^{\mathrm{T}}\right)^{-1} \mathbf{h}_{n},
$$

where $\bar{v}=\frac{1}{L} \cdot \sum_{n=0}^{L-1} v_{n}$ is the average variance of the symbols $x_{n}$ and $\overline{\mathbf{V}}=\frac{1}{L} \cdot \sum_{n=0}^{L}-1 \mathbf{V}_{n}$. This choice minimizes the average MSE $\frac{1}{L} \cdot \sum_{n=0}^{L-1} \mathrm{E}\left\{\left|\hat{x}_{n}-x_{n}\right|^{2}\right\}$ if $\kappa$ is chosen suitably, which can be shown using the orthogonality principle. The choice $\kappa=$ $1 /\left(1+(1-\bar{v}) \mathbf{f}^{\mathrm{T}} \mathbf{h}_{n}\right)$ yields $\frac{1}{L} \cdot \sum_{n=0}^{L-1} \mathrm{E}\left\{\mathbf{y}_{n}\left(\hat{x}_{n}-x_{n}\right)\right\}=\mathbf{0}_{W}$. The solution (37) is MMSE-optimal, i.e., it minimizes the MSE $\mathrm{E}\left\{\left|\hat{x}_{n}-x_{n}\right|^{2}\right\}$, whenever all $v_{n}$ are constant, e.g., in case all $v_{n}$ are 1 (initial equalization step) or all $v_{n}$ are 0 (convergence of turbo equalization).

The term $\overline{\mathbf{V}}$ can be approximated with $\bar{v} \mathbf{I}_{M_{h}+W}$ by neglecting the boundary effects for time indices $n$ smaller than $M_{h}+W_{2}$ and larger than $L-W_{1}-1$, which simplifies (37) to

$$
\mathbf{f}=\left(\sigma^{2} \mathbf{I}_{W}+\bar{v} \mathbf{H}_{n} \mathbf{H}_{n}^{\mathrm{T}}\right)^{-1} \mathbf{h}_{n} .
$$

Using this coefficient vector, the estimates $\hat{x}_{n}$ are given by

$$
\begin{aligned}
\hat{x}_{n}=\kappa \cdot\left(\mathbf{f}^{\mathrm{T}}\left(\mathbf{y}_{n}-\mathbf{H}_{n} \boldsymbol{\mu}_{n}\right)+\mu_{n} \mathbf{f}^{\mathrm{T}} \mathbf{h}_{n}\right), \\
\kappa=1 /\left(1+(1-\bar{v}) \mathbf{f}^{\mathrm{T}} \mathbf{h}_{n}\right) .
\end{aligned}
$$

The statistics $\mathrm{E}\left\{e_{n}\right\}$ and $\operatorname{Var}\left\{e_{n}\right\}$ of the estimation error $e_{n}=$ $\hat{x}_{n}-x_{n}$ are given by

$$
\begin{aligned}
\mathrm{E}\left\{e_{n}\right\} & =\mathrm{E}\left\{\hat{x}_{n}\right\} \\
& =\kappa \cdot\left(\mathbf{f}^{\mathrm{T}}\left(\mathrm{E}\left\{\mathbf{y}_{n}\right\}-\mathbf{H}_{n} \boldsymbol{\mu}_{n}\right)+\mu_{n} \mathbf{f}^{\mathrm{T}} \mathbf{h}_{n}\right)=0,
\end{aligned}
$$


TABLE VI

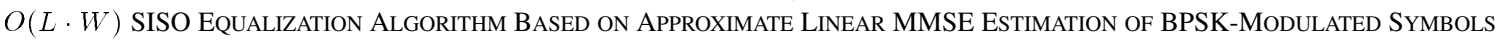

\begin{tabular}{l}
\hline INPUT \\
\hline extrinsic LLRs $\lambda_{e x t}^{\prime}\left(c_{n}\right)$ for $n=0,1, \ldots, N-1$ \\
\hline INITIALIZATION \\
\hline compute $\mu_{n}=\tanh \left(\lambda_{e x t}^{\prime}\left(c_{n}\right) / 2\right)$ and $v_{n}=1-\left|\mu_{n}\right|^{2}$ for $n=0,1, \ldots, N-1$ \\
compute $\bar{v}=\frac{1}{L} \cdot \sum_{n=0}^{L-1} v_{n}$ \\
compute $\mathbf{f}=\left(\sigma^{2} \mathbf{I}_{W}+\bar{v} \mathbf{H}_{0} \mathbf{H}_{0}^{\mathrm{T}}\right)^{-1} \mathbf{h}_{0}$ \\
compute $s=\mathbf{f}^{\mathrm{T}} \mathbf{h}_{0}$ \\
compute $\kappa=2 /(1-\bar{v} s)$ \\
\hline APPROXIMATE LINEAR MMSE ESTIMATION \\
\hline FOR $n=0$ TO $N-1$ DO \\
$\quad \lambda_{e x t}\left(c_{n}\right)=\kappa \cdot\left(\mathbf{f}^{\mathrm{T}}\left(\mathbf{y}_{n}-\mathbf{H}_{n} \boldsymbol{\mu}_{n}\right)+\mu_{n} \cdot s\right)$ \\
END
\end{tabular}

OUTPUT

extrinsic LLRs $\lambda_{e x t}\left(c_{n}\right)$ for $n=0,1, \ldots, N-1$

$$
\begin{aligned}
\operatorname{Var}\left\{e_{n}\right\}= & \operatorname{Var}\left\{\hat{x}_{n}\right\}+\operatorname{Var}\left\{x_{n}\right\}-2 \operatorname{Cov}\left\{\hat{x}_{n}, x_{n}\right\} \\
= & \kappa^{2} \mathbf{f}^{\mathrm{T}} \operatorname{Cov}\left\{\mathbf{y}_{n}, \mathbf{y}_{n}\right\} \mathbf{f} \\
& +1-2 \kappa \mathbf{f}^{\mathrm{T}} \operatorname{Cov}\left\{\mathbf{y}_{n}, x_{n}\right\} \\
= & \kappa^{2} \mathbf{f}^{\mathrm{T}}\left(\sigma^{2} \mathbf{I}_{W}+\mathbf{H}_{n} \mathbf{V}_{n} \mathbf{H}_{n}^{\mathrm{T}}\right. \\
& \left.+\left(1-v_{n}\right) \mathbf{h}_{n} \mathbf{h}_{n}^{\mathrm{T}}\right) \mathbf{f}+1-2 \kappa \mathbf{f}^{\mathrm{T}} \mathbf{h}_{n}
\end{aligned}
$$

recalling that $\mathrm{E}\left\{x_{n}\right\}=0$ and $\operatorname{Var}\left\{x_{n}\right\}=1$ holds. Using the Gaussian assumption on $p\left(e_{n}\right)$ and (13), the extrinsic LLRs $\lambda_{\text {ext }}\left(c_{n}\right)$ can be obtained as follows:

$$
\begin{aligned}
\lambda_{\text {ext }}\left(c_{n}\right) & =\ln \frac{\exp \left(-\left(\hat{x}_{n}-+1\right)^{2} /\left(2 \operatorname{Var}\left\{e_{n}\right\}\right)\right)}{\exp \left(-\left(\hat{x}_{n}--1\right)^{2} /\left(2 \operatorname{Var}\left\{e_{n}\right\}\right)\right)} \\
& =\frac{2 \hat{x}_{n}}{\operatorname{Var}\left\{e_{n}\right\}} .
\end{aligned}
$$

A simplified way to compute $\lambda_{\text {ext }}\left(c_{n}\right)$ is to approximate $p\left(e_{n}\right)$ with a Gaussian distribution with time invariant statistics. The mean of this distribution is set to 0 , since $\mathrm{E}\left\{e_{n}\right\}$ is always 0 . The variance $\overline{\operatorname{Var}\left\{e_{n}\right\}}$ of this distribution is set to the average estimation error variance over all time indices $n=0,1, \ldots, L-$ 1

$$
\begin{aligned}
\overline{\operatorname{Var}\left\{e_{n}\right\}}= & \frac{1}{L} \cdot \sum_{n=0}^{L-1} \operatorname{Var}\left\{e_{n}\right\} \\
= & \kappa^{2} \mathbf{f}^{\mathrm{T}}\left(\sigma^{2} \mathbf{I}_{W}+\mathbf{H}_{n} \overline{\mathbf{V}} \mathbf{H}_{n}^{\mathrm{T}}+(1-\bar{v}) \mathbf{h}_{n} \mathbf{h}_{n}^{\mathrm{T}}\right) \mathbf{f} \\
& +1-2 \kappa \mathbf{f}^{\mathrm{T}} \mathbf{h}_{n} \\
= & \kappa s+1-2 \kappa s=1-\kappa s, \quad s=\mathbf{f}^{\mathrm{T}} \mathbf{h}_{n},
\end{aligned}
$$

where the last line follows from the approximation $\overline{\mathbf{V}} \approx$ $\bar{v} \mathbf{I}_{M_{h}+W}$. The extrinsic LLRs $\lambda_{\text {ext }}\left(c_{n}\right)$ are then given by

$$
\begin{aligned}
\lambda_{\text {ext }}\left(c_{n}\right) & =\ln \frac{\exp \left(-\left(\hat{x}_{n}-+1\right)^{2} /\left(2 \overline{\operatorname{Var}\left\{e_{n}\right\}}\right)\right)}{\exp \left(-\left(\hat{x}_{n}--1\right)^{2} /\left(2 \overline{\operatorname{Var}\left\{e_{n}\right\}}\right)\right)} \\
& =\frac{2 \hat{x}_{n}}{\overline{\operatorname{Var}\left\{e_{n}\right\}}}
\end{aligned}
$$

$$
\begin{aligned}
& =\frac{2 \kappa\left(\mathbf{f}^{\mathrm{T}}\left(\mathbf{y}_{n}-\mathbf{H}_{n} \boldsymbol{\mu}_{n}\right)+\mu_{n} s\right)}{1-\kappa s} \\
& =\frac{2\left(\mathbf{f}^{\mathrm{T}}\left(\mathbf{y}_{n}-\mathbf{H}_{n} \boldsymbol{\mu}_{n}\right)+\mu_{n} s\right)}{1-\bar{v} s} .
\end{aligned}
$$

Note that this simplification of the LLR calculation assigns that same reliability to each output LLR $\lambda_{\text {ext }}\left(c_{n}\right)$. The complete SISO equalization based on approximate linear MMSE estimation of BPSK-modulated symbols is summarized in Table VI. The complexity order of this SISO equalization algorithm is $O(L \cdot W)$.

The BER performance of turbo equalization using approximate linear MMSE estimation is exhibited in Fig. 11 for the same block length $K=510$ as in Fig. 8, which depicts the performance of turbo equalization using exact linear MMSE estimation. Comparing the two figures indicates that the performance degradation due to the imposed approximations is small. Both estimation algorithms attain the BER performance of the FEC decoder when no ISI is introduced in the channel. Recall that SISO equalization based on exact linear MMSE estimation has the complexity order $O\left(L \cdot W^{2}\right)$ as shown in Table IV, whereas SISO equalization based on approximate linear MMSE estimation has the complexity order $O(L \cdot W)$.

\section{Approximate Linear MMSE Estimation in the Frequency Domain}

Consider the following linear MMSE estimator:

$$
\hat{\mathbf{x}}=\mathrm{E}\{\mathbf{x}\}+\operatorname{Cov}\{\mathbf{x}, \mathbf{y}\} \operatorname{Cov}\{\mathbf{y}, \mathbf{y}\}^{-1}(\mathbf{y}-\mathrm{E}\{\mathbf{y}\}),
$$

which, when $\hat{x}_{n}$ does not depend on the corresponding symbol statistics $\mu_{n}$ and $v_{n}$, yields

$$
\begin{aligned}
\hat{x}_{n}=\mathbf{h}_{n}^{\mathrm{T}}\left(\sigma^{2} \mathbf{I}_{L}+\mathbf{H} \mathbf{V} \mathbf{H}^{\mathrm{T}}+\left(1-v_{n}\right) \mathbf{h}_{n} \mathbf{h}_{n}^{\mathrm{T}}\right)^{-1} & \\
& \times\left(\mathbf{y}-\mathbf{H} \boldsymbol{\mu}+\mu_{n} \mathbf{h}_{n}\right),
\end{aligned}
$$

where $\boldsymbol{\mu}=\left(\mu_{0} \mu_{1} \ldots \mu_{L-1}\right)^{\mathrm{T}}, \mathbf{V}=\left(v_{0} v_{1} \ldots v_{L-1}\right)^{\mathrm{T}}$, and $\mathbf{h}_{n}=\mathbf{H}[0: L-1, n]$ is (here) the $n$th column of $\mathbf{H}$. By 
TABLE VII

$O(L \cdot \ln L)$ SISO EqualizATION ALGORITHM BASED ON APPROXIMATE LiNEAR MMSE ESTIMATION OF BPSK-MODULATED SYMBOLS IN THE FREQUENCY DOMAIN

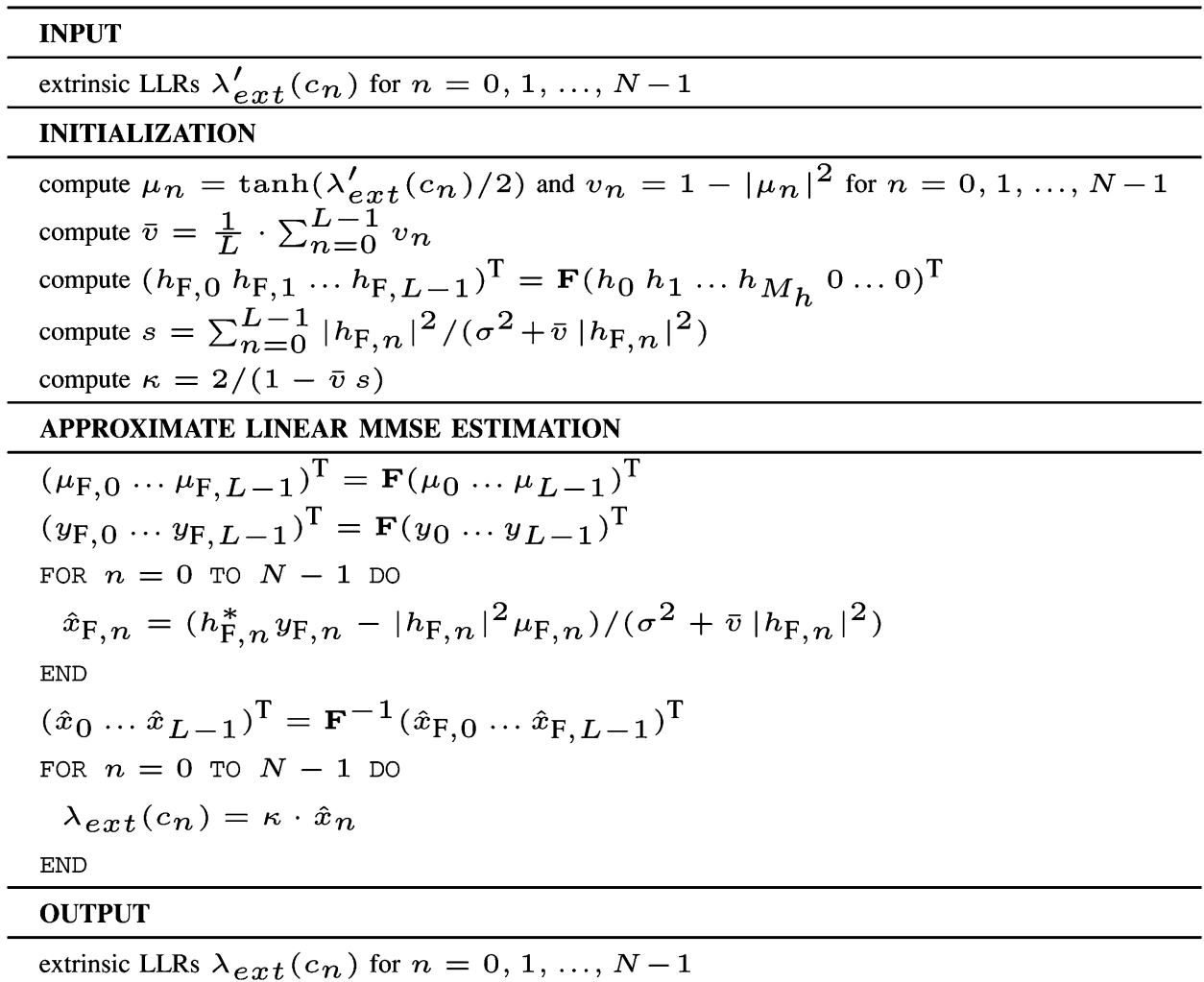

considering the following approximation, which is similar to that in Section IV-C, i.e., it minimizes the average MSE $\frac{1}{L}$. $\sum_{n=0}^{L-1} \mathrm{E}\left\{\left(\hat{x}_{n}-x_{n}\right)^{2}\right\}:$

$$
\begin{aligned}
\hat{x}_{n} & =\mathbf{h}_{n}^{\mathrm{T}}\left(\boldsymbol{\Sigma}+(1-\bar{v}) \mathbf{h}_{n} \mathbf{h}_{n}^{\mathrm{T}}\right)^{-1}\left(\mathbf{y}-\mathbf{H} \boldsymbol{\mu}+\mu_{n} \mathbf{h}_{n}\right) \\
& =\frac{\mathbf{h}_{n}^{\mathrm{T}} \boldsymbol{\Sigma}^{-1}\left(\mathbf{y}-\mathbf{H} \boldsymbol{\mu}+\mu_{n} \mathbf{h}_{n}\right)}{1+(1-\bar{v}) \mathbf{h}_{n}^{\mathrm{T}} \boldsymbol{\Sigma}^{-1} \mathbf{h}_{n}},
\end{aligned}
$$

where $\boldsymbol{\Sigma}=\sigma^{2} \mathbf{I}_{L}+\bar{v} \mathbf{H} \mathbf{H}^{\mathrm{T}}$, we see that the term $\mathbf{h}_{n}^{\mathrm{T}} \boldsymbol{\Sigma}^{-1} \mathbf{h}_{n}$ is constant for all $n$ given a time invariant ISI channel and a system matrix $\mathbf{H}$ constructed under a periodic extension. Using a cyclic prefix for transmission, i.e., $\left(x_{L-2} x_{L-1} x_{0} x_{1} \ldots x_{L-1}\right)^{\mathrm{T}}, \mathbf{H}$ becomes a circulant matrix. The subsequence $\left(x_{L-2} \ldots x_{L-1}\right)^{\mathrm{T}}$ transmitted prior to $\mathbf{x}$ is the so-called cyclic prefix [94], which in general consists of the $M_{h}$ last symbols of $\mathbf{x}$ given a memory- $M_{h}$ ISI channel. Using the constant term $s=\mathbf{h}_{n}^{\mathrm{T}} \boldsymbol{\Sigma}^{-1} \mathbf{h}_{n}$, the estimates $\hat{x}_{n}$ are given by

$$
\hat{x}_{n}=\left(\mathbf{h}_{n}^{\mathrm{T}} \boldsymbol{\Sigma}^{-1}(\mathbf{y}-\mathbf{H} \boldsymbol{\mu})+\mu_{n} s\right) /(1+(1-\bar{v}) s),
$$

which can be combined to

$$
\hat{\mathbf{x}}=\left(\mathbf{H}^{\mathrm{T}} \boldsymbol{\Sigma}^{-1}(\mathbf{y}-\mathbf{H} \boldsymbol{\mu})+\boldsymbol{\mu} s\right) /(1+(1-\bar{v}) s) .
$$

The complexity of this calculation can be reduced to $O(L \cdot \ln L)$ by calculating (42) efficiently in the frequency domain, since $\mathbf{H}$ is a circulant matrix, i.e., its columns are cyclic shifts of the first column $\mathbf{h}_{0}=\left(h_{0} h_{1} \ldots h_{M_{h}} 0 \ldots 0\right)^{\mathrm{T}}$. It follows that $\mathbf{H}$ is equal to $\mathbf{F} \operatorname{Diag}\left\{\mathbf{h}_{\mathrm{F}}\right\} \mathbf{F}^{-1}$, where $\mathbf{F}$ is the $L \times L$ DFT matrix and

$$
\mathbf{h}_{\mathrm{F}}=\left(h_{\mathrm{F}, 0} h_{\mathrm{F}, 1} \ldots h_{\mathrm{F}, L-1}\right)^{\mathrm{T}}=\mathbf{F} \mathbf{h}_{0}
$$

is the spectrum of $\mathbf{h}_{0}$. Applying this relationship to $\boldsymbol{\Sigma}^{-1}$ yields

$$
\begin{aligned}
\boldsymbol{\Sigma}^{-1}= & \left(\sigma^{2} \mathbf{I}_{L}\right. \\
& \left.+\bar{v} \mathbf{F} \operatorname{Diag}\left\{\mathbf{h}_{\mathrm{F}}\right\} \mathbf{F}^{-1}\left(\mathbf{F} \operatorname{Diag}\left\{\mathbf{h}_{\mathrm{F}}\right\} \mathbf{F}^{-1}\right)^{\mathrm{H}}\right)^{-1} \\
= & \left(\sigma^{2} \mathbf{I}_{L}+\bar{v} \mathbf{F} \operatorname{Diag}\left\{\mathbf{h}_{\mathrm{F}}\right\} \operatorname{Diag}\left\{\mathbf{h}_{\mathrm{F}}\right\}^{*} \mathbf{F}^{-1}\right)^{-1} \\
= & \mathbf{F}^{-1}\left(\sigma^{2} \mathbf{I}_{L}+\bar{v} \operatorname{Diag}\left\{\mathbf{h}_{\mathrm{F}}\right\} \operatorname{Diag}\left\{\mathbf{h}_{\mathrm{F}}\right\}^{*}\right)^{-1} \mathbf{F},
\end{aligned}
$$

where $\mathbf{H}^{\mathrm{T}}$ was replaced by $\mathbf{H}^{\mathrm{H}}$ in order that the properties $\left(\mathbf{F}^{-1}\right)^{\mathrm{H}}=\mathbf{F} / L$ and $\mathbf{F}^{\mathrm{H}}=L \cdot \mathbf{F}^{-1}$ of the DFT matrix can be applied. The estimate vector $\hat{\mathbf{x}}$ is finally given by (43), at the bottom of the next page. It will be exact whenever all symbol variances $v_{n}$ are identical, e.g., in the initial equalization step in turbo equalization, where all $v_{n}$ are 1 . The constant $s=$ $\mathbf{h}_{n}^{\mathrm{T}} \boldsymbol{\Sigma}^{-1} \mathbf{h}_{n}$ can be calculated using $\mathbf{F D i a g}\left\{\mathbf{h}_{\mathrm{F}}\right\} \mathbf{F}^{-1} \mathbf{1}_{L}$ :

$$
\begin{aligned}
s= & \mathbf{1}_{L}^{\mathrm{T}} \operatorname{Diag}\left\{\mathbf{h}_{\mathrm{F}}\right\}^{*}\left(\sigma^{2} \mathbf{I}_{L}\right. \\
& \left.\quad+\bar{v} \operatorname{Diag}\left\{\mathbf{h}_{\mathrm{F}}\right\} \operatorname{Diag}\left\{\mathbf{h}_{\mathrm{F}}\right\}^{*}\right)^{-1} \operatorname{Diag}\left\{\mathbf{h}_{\mathrm{F}}\right\} \mathbf{1}_{L} \\
= & \sum_{n=0}^{L-1} \frac{h_{\mathrm{F}, n} h_{\mathrm{F}, n}^{*}}{\sigma^{2}+\bar{v} h_{\mathrm{F}, n} h_{\mathrm{F}, n}^{*}} .
\end{aligned}
$$


TABLE VIII

$O\left(L \cdot W^{2}\right)$ SISO EQUaLIZATION ALgORITHM BASED ON LiNEAR MMSE ESTIMATION OF PSK- OR QAM-MOdULATED SYMBOLS. THE OPERATOR $:=$ DENOTES THAT THE VALUE OF THE LEFT-HAND ARGUMENT Is REPLACED BY THAT OF THE RIGHT-HAND ARGUMENT

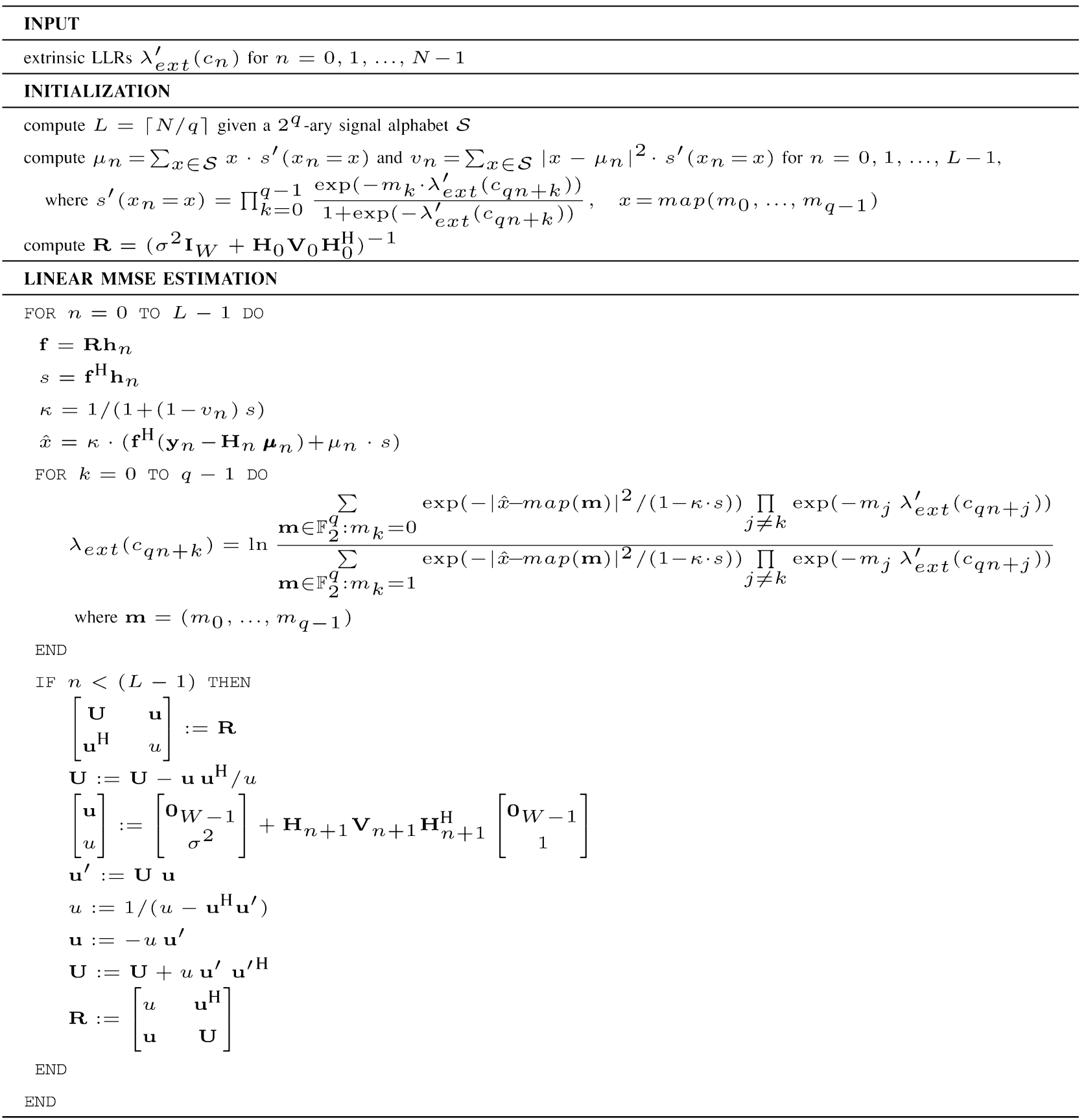

\section{OUTPUT}

extrinsic LLRs $\lambda_{e x t}\left(c_{n}\right)$ for $n=0,1, \ldots, N-1$

The complexity of the actual estimation part is only $O(L)$ and that of the complete equalization algorithm is $O(L \cdot \ln L)$, using the fast Fourier transform [95]. The extrinsic LLRs $\lambda_{\text {ext }}\left(c_{n}\right)$ are calculated as in Section IV-C. A detailed study of the complexity of estimation algorithms working either in the time or in the frequency domain has been carried out in [96].

The complete SISO equalization algorithm based on approximate linear MMSE estimation of BPSK-modulated symbols in

$$
\hat{\mathbf{x}}=\frac{\mathbf{F}^{-1} \operatorname{Diag}\left\{\mathbf{h}_{\mathrm{F}}\right\}^{*}\left(\sigma^{2} \mathbf{I}_{L}+\bar{v} \operatorname{Diag}\left\{\mathbf{h}_{\mathrm{F}}\right\} \operatorname{Diag}\left\{\mathbf{h}_{\mathrm{F}}\right\}^{*}\right)^{-1}\left(\mathbf{F} \mathbf{y}-\operatorname{Diag}\left\{\mathbf{h}_{\mathrm{F}}\right\} \mathbf{F} \boldsymbol{\mu}\right)+\boldsymbol{\mu} s}{1+(1-\bar{v}) s} .
$$


TABLE IX

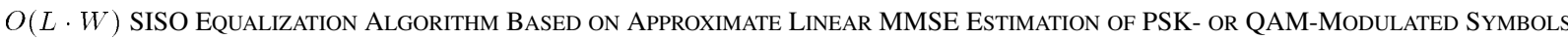

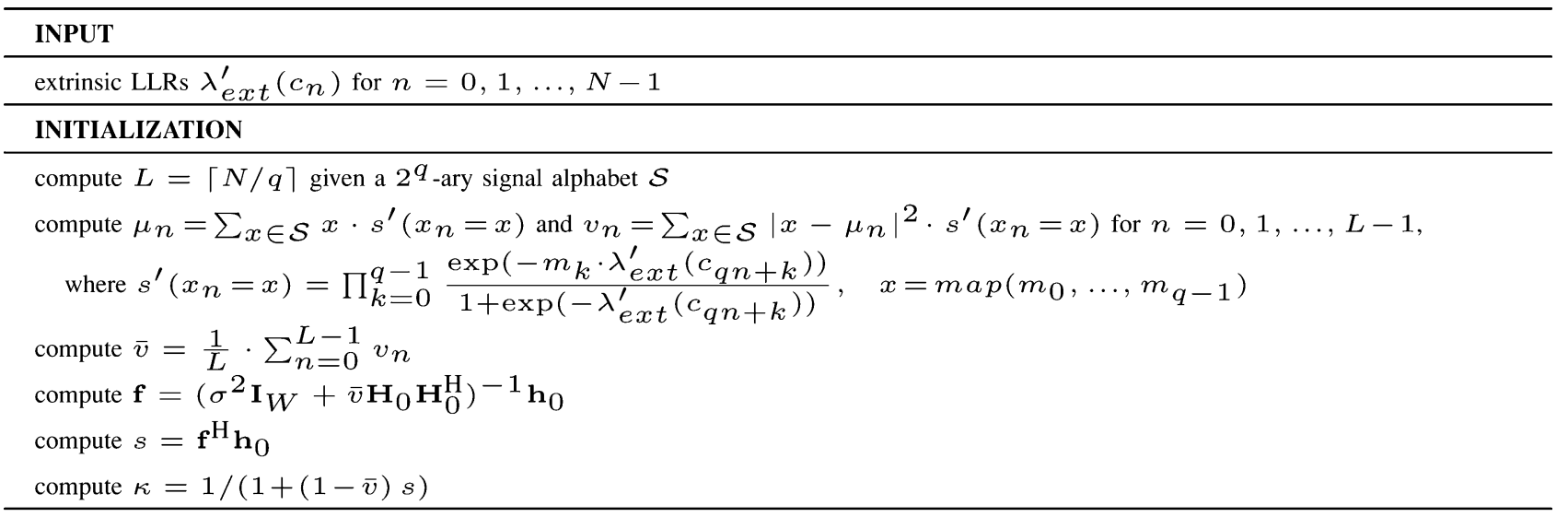

\section{APPROXIMATE LINEAR MMSE ESTIMATION}

FOR $n=0$ TO $L-1$ DO

$\hat{x}=\kappa \cdot\left(\mathbf{f}^{\mathrm{H}}\left(\mathbf{y}_{n}-\mathbf{H}_{n} \boldsymbol{\mu}_{n}\right)+\mu_{n} \cdot s\right)$

FOR $k=0$ TO $q-1$ DO

$\lambda_{e x t}\left(c_{q n+k}\right)=\ln \frac{{\mathbf{m} \in \mathbb{F}_{2}^{q}: m_{k}=0}_{\sum_{\mathbf{m} \in \mathbb{F}_{2}^{q}: m_{k}=1} \exp \left(-\left|\hat{x}_{n}-m a p(\mathbf{m})\right|^{2} /(1-\kappa s)\right)}^{\exp \left(-\left|\hat{x}_{n}-\operatorname{map}(\mathbf{m})\right|^{2} /(1-\kappa s)\right)} \prod_{j \neq k} \exp \left(-m_{j} \lambda_{e x t}^{\prime}\left(c_{q n+j}\right)\right)}{\exp \left(-m_{j} \lambda_{e x t}^{\prime}\left(c_{q n+j}\right)\right)}$

where $\mathbf{m}=\left(m_{0}, \ldots, m_{q-1}\right)$

END

END

\section{OUTPUT}

extrinsic LLRs $\lambda_{e x t}\left(c_{n}\right)$ for $n=0,1, \ldots, N-1$

the frequency domain is summarized in Table VII. The BER performance of turbo equalization using this estimation algorithm is identical to that in Section IV-C, where approximate linear MMSE estimation of BPSK-modulated symbols in the time domain was applied. The frequency domain approach can perform slightly better than the time domain counterpart in Section IV-C, since the entire received sequence $\mathbf{y}$ is used to estimate each symbol $x_{n}$. However, the improvement is usually small as long as the window parameter $W$ is chosen sufficiently long.

\section{E. Linear MMSE Estimation of PSK- or QAM-Modulated Symbols}

Another approximation is to simply discard the pseudovariances $\psi_{n}$ while computing $\hat{x}_{n}$, i.e., the transmitted symbols $x_{n}$ are assumed to be circularly symmetric. By setting $\psi_{n}$ to 0 for all $n$, the calculation of the estimates $\hat{x}_{n}$ in (29) simplifies greatly, since $\boldsymbol{\Psi}_{n}$ and $\mathbf{v}_{n}$ vanish for all $n$ and $\mathbf{u}_{n}$ is given by $\mathbf{h}_{n}^{\mathrm{H}} \tilde{\boldsymbol{\Sigma}}_{n}$

$$
\hat{x}_{n}=\mathbf{h}_{n}^{\mathrm{H}} \tilde{\boldsymbol{\Sigma}}_{n}^{-1}\left(\mathbf{y}_{n}-\mathbf{H}_{n} \boldsymbol{\mu}_{n}+\mu_{n} \mathbf{h}_{n}\right),
$$

where $\tilde{\boldsymbol{\Sigma}}_{n}=\boldsymbol{\Sigma}_{n}+\left(1-v_{n}\right) \mathbf{h}_{n} \mathbf{h}_{n}^{\mathrm{H}}$ and $\boldsymbol{\Sigma}_{n}=\sigma^{2} \mathbf{I}_{W}+$ $\mathbf{H}_{n} \mathbf{V}_{n} \mathbf{H}_{n}^{\mathrm{H}}$. The expression $\tilde{\boldsymbol{\Sigma}}_{n}^{-1} \mathbf{h}_{n}$ is equal to $\boldsymbol{\Sigma}_{n}^{-1} \mathbf{h}_{n} /(1+$ $\left.\left(1-v_{n}\right) \mathbf{h}_{n}^{\mathrm{H}} \boldsymbol{\Sigma}_{n}^{-1} \mathbf{h}_{n}\right)$, which can be shown using Woodbury's identity. It follows that the estimates $\hat{x}_{n}$ can be computed as follows:

$$
\begin{aligned}
\hat{x}_{n} & =\frac{\mathbf{f}_{n}^{\mathrm{H}}\left(\mathbf{y}_{n}-\mathbf{H}_{n} \boldsymbol{\mu}_{n}\right)+\mu_{n} \cdot s_{n}}{1+\left(1-v_{n}\right) s_{n}}, \\
\mathbf{f}_{n} & =\boldsymbol{\Sigma}_{n}^{-1} \mathbf{h}_{n}, \quad s_{n}=\mathbf{f}_{n}^{\mathrm{H}} \mathbf{h}_{n} .
\end{aligned}
$$

The computation of the extrinsic LLRs $\lambda_{\text {ext }}\left(c_{n}\right)$ can be shown to be as described in the first equation at the bottom of the next page, where $\mathbf{m}=\left(m_{0}, \ldots, m_{q-1}\right)$. The complete order $O\left(L \cdot W^{2}\right)$ SISO equalization algorithm based on linear MMSE estimation of PSK- or QAM-modulated symbols is summarized in Table VIII.

The BER performance of turbo equalization using the linear MMSE estimation of PSK- or QAM-modulated symbols is studied at the end of the next section, which introduces an even simpler MMSE estimation algorithm. 
TABLE X

$O(L \cdot \ln L)$ SISO EQUALIZATION ALGORITHM BASED ON APPROXIMATE LINEAR MMSE ESTIMATION OF PSK- OR QAM-MODULATED SYMBOLS IN THE FREQUENCY DOMAIN

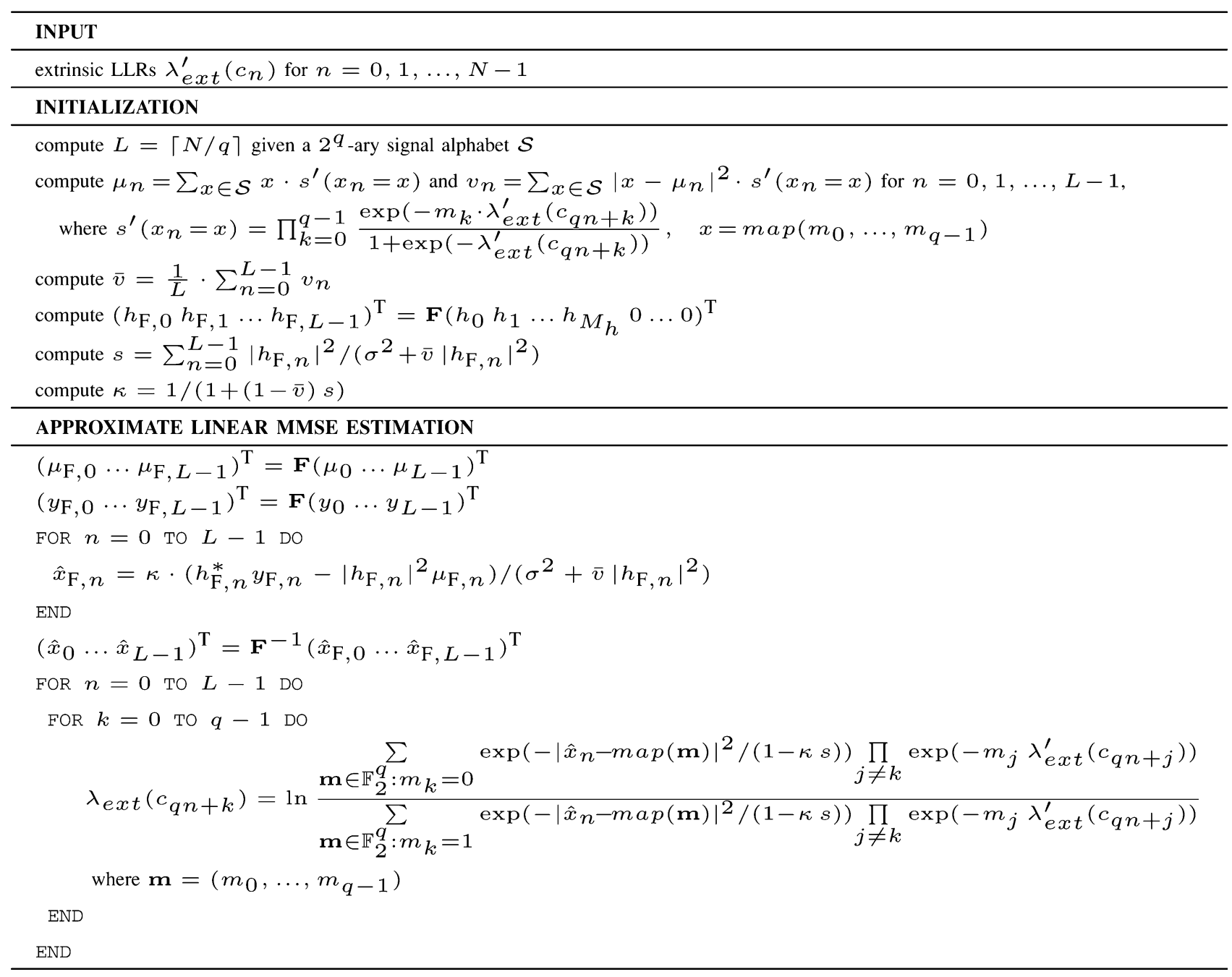

\section{OUTPUT}

extrinsic LLRs $\lambda_{\text {ext }}\left(c_{n}\right)$ for $n=0,1, \ldots, N-1$

\section{F. Approximate Linear MMSE Estimation of PSK- or QAM-Modulated Symbols}

An approximate linear MMSE estimation of PSK- or QAMmodulated symbols algorithm follows from using a time-invariant coefficient vector to compute the estimates $\hat{x}_{n}$,

$$
\begin{aligned}
\hat{x}_{n} & =\kappa \cdot\left(\mathbf{f}^{\mathrm{H}}\left(\mathbf{y}_{n}-\mathbf{H}_{n} \boldsymbol{\mu}_{n}\right)+\mu_{n} s\right), \\
\mathbf{f} & =\left(\sigma^{2} \mathbf{I}_{W}+\bar{v} \mathbf{H}_{n} \mathbf{H}_{n}^{\mathrm{H}}\right)^{-1} \mathbf{h}_{n},
\end{aligned}
$$

where $s=\mathbf{f}^{\mathrm{H}} \mathbf{h}_{n}$ and $\kappa=1+(1-\bar{v}) s$. This solution has a complexity order of $O(W)$ per time step $n$ similar to the approximate linear MMSE estimation algorithm for BPSK-modulated symbols summarized in Table VI. It minimizes the average MSE $\frac{1}{L} \cdot \sum_{n=0}^{L-1} \mathrm{E}\left\{\left(\hat{x}_{n}-x_{n}\right)^{2}\right\}$ provided that the average pseudovariance $\bar{\psi}=\frac{1}{L} \cdot \sum_{n=0}^{L-1} \psi_{n}$ vanishes. For computing the extrinsic LLRs $\lambda_{\text {ext }}\left(c_{n}\right)$, the same approximate calculation of the estimation error statistics as in Section IV-C is applied, i.e., the PDF $p\left(e_{n}\right)$ is approximated with a Gaussian distribution with time invariant mean $\overline{\mathrm{E}\left\{e_{n}\right\}}=0$, variance $\overline{\operatorname{Var}\left\{e_{n}\right\}}=1-\kappa s$,

$$
\lambda_{\text {ext }}\left(c_{q n+k}\right)=\ln \frac{\sum_{\mathbf{m} \in \mathbb{F}_{2}^{q}: m_{k}=0} \exp \left(-\frac{\left|\hat{x}_{n}-\operatorname{map}(\mathbf{m})\right|^{2}}{1-s_{n} /\left(1+\left(1-v_{n}\right) s_{n}\right)}\right) \prod_{j \neq k} \exp \left(-m_{j} \lambda_{\text {ext }}^{\prime}\left(c_{q n+j}\right)\right)}{\sum_{\mathbf{m} \in \mathbb{F}_{2}^{q}: m_{k}=1} \exp \left(-\frac{\left|\hat{x}_{n}-\operatorname{map}(\mathbf{m})\right|^{2}}{1-s_{n} /\left(1+\left(1-v_{n}\right) s_{n}\right)}\right) \prod_{j \neq k} \exp \left(-m_{j} \lambda_{\text {ext }}^{\prime}\left(c_{q n+j}\right)\right)},
$$




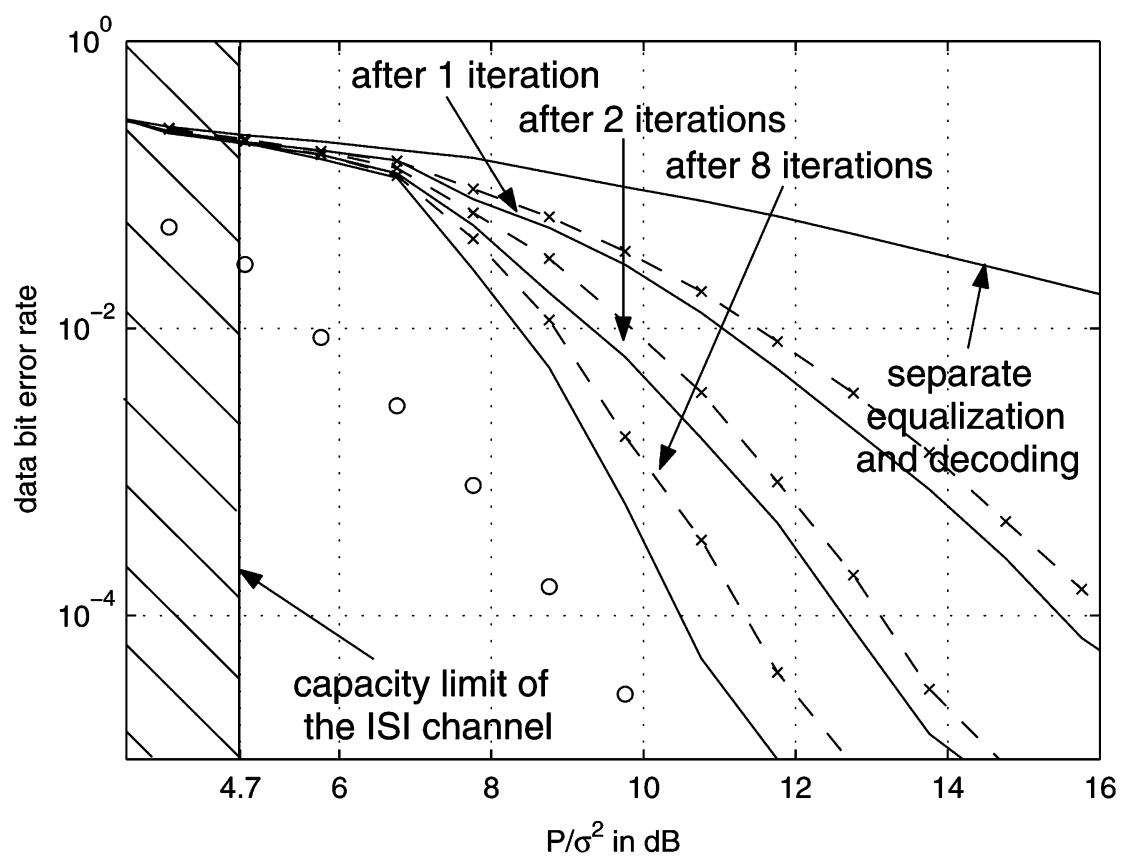

Fig. 12. Performance of turbo equalization for the communication system described in Section III-A using (approximate) linear MMSE estimation. The BER performance is plotted for separate equalization and decoding as well as after 1,2, or 8 iterations, where the solid lines correspond to the order $O\left(L \cdot W^{2}\right)$ linear MMSE algorithm algorithm summarized in Table VIII and the dashed lines with crosses correspond to the order $O(L \cdot W)$ approximate linear MMSE algorithm summarized in Table IX. The dotted line corresponds to the BER performance of bit-interleaved coded modulation given an ISI-free channel after 8 demapping-decoding iterations. The considered blocklength is $K=510(N=1024$ and $L=342)$. An S-random interleaver with $\mathrm{S}=16$ is applied.
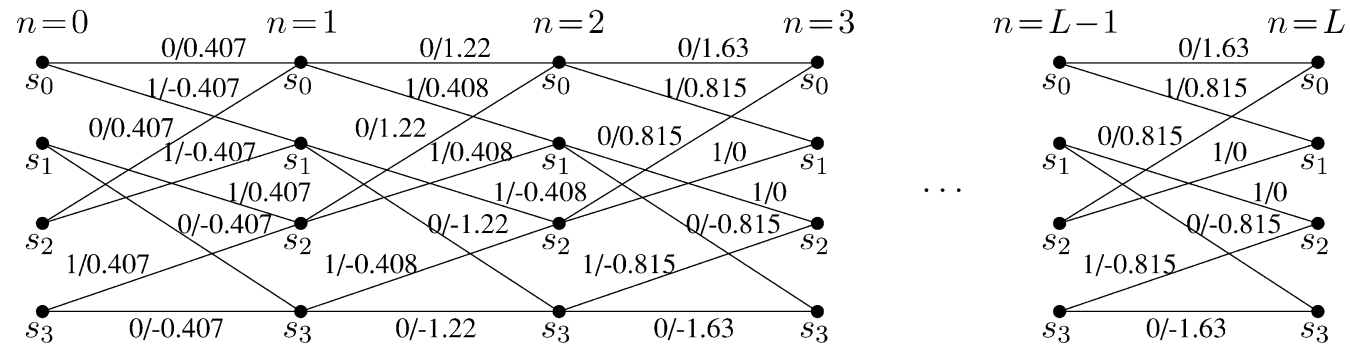

Fig. 13. Combined trellis of a length-3 ISI channel given by the coefficients $h_{0}=0.407, h_{1}=0.815$, and $h_{2}=0.407$ and the precoder $\tilde{c}_{n}=c_{n}+\tilde{c}_{n-1}$ under the termination assumption. Note that compared to the trellis in Fig. 3, only the input $x_{n}$ in the labels $x_{n} / v_{n}$ has changed.

and pseudovariance $\overline{\operatorname{Cov}\left\{e_{n}, e_{n}^{*}\right\}}=\frac{1}{L} \cdot \sum_{n=0}^{L-1} \operatorname{Cov}\left\{e_{n}, e_{n}^{*}\right\}$. The latter is again ignored, which yields the formula to calculate the LLRs $\lambda_{\text {ext }}\left(c_{n}\right)$ as shown in the equation at the bottom of the page, where $\mathbf{m}=\left(m_{0}, \ldots, m_{q-1}\right)$. The complete order $O(L \cdot W)$ SISO equalization algorithm based on approximate linear MMSE estimation of PSK- or QAM-modulated symbols is summarized in Table IX.

The BER performance of turbo equalization based on (approximate) linear MMSE estimation of PSK- or QAM-modulated symbols is exhibited in Fig. 12 for the same block length
$K=510$ as in Fig. 9, which depicts the performance of turbo equalization using exact MMSE estimation. Both the order $O\left(L \cdot W^{2}\right)$ linear MMSE algorithm summarized in Table VIII and the order $O(L \cdot W)$ approximate linear MMSE algorithm summarized in Table IX are considered. Comparing the two figures indicates that the performance degradation due to the imposed approximations is small, but not negligible. For separate equalization and decoding, all three solutions are identical and, thus, yield the same BER performance.

$$
\lambda_{\text {ext }}\left(c_{q n+k}\right)=\ln \frac{\sum_{\mathbf{m} \in \mathbb{F}_{2}^{q}: m_{k}=0} \exp \left(-\frac{\left|\hat{x}_{n}-\operatorname{map}(\mathbf{m})\right|^{2}}{1-\kappa s}\right) \prod_{j \neq k} \exp \left(-m_{j} \lambda_{\text {ext }}^{\prime}\left(c_{q n+j}\right)\right)}{\sum_{\mathbf{m} \in \mathbb{F}_{2}^{q}: m_{k}=1} \exp \left(-\frac{\left|\hat{x}_{n}-\operatorname{map}(\mathbf{m})\right|^{2}}{1-\kappa s}\right) \prod_{j \neq k} \exp \left(-m_{j} \lambda_{\text {ext }}^{\prime}\left(c_{q n+j}\right)\right)},
$$




\section{PRECODING}

When considering the concatenation of a channel code with an interleaver followed by an ISI channel as a serially concatenated convolutional code, it is clear that the inner code in this defacto turbo-code is the rate-one convolutional code over the reals provided by the ISI channel. With a finite delay-spread, this inner code typically has a poor distance spectrum. One method of improving the performance of this inner coder is through the use of a recursive precoder which, in effect, makes the inner-code, of the constructed turbo-code, recursive.

We now consider the use of precoders in the transmitter of Fig. 1, i.e., where $q$-tuples $\mathbf{c}_{n}$ of code bits $c_{n}$ are precoded to the $q$-tuples $\tilde{\mathbf{c}}_{n}$ using a memory- $M_{p}$ state-space model. The BER performance of ML decoding with perfect interleaving but no precoding and an ISI channel is lower bounded by the ML decoding performance of the FEC decoder given an ISI-free channel at the same SNR. This lower bound can be seen in Figs. 6, 8, 9, 10, 11, and 12. Clearly, the decoding performance is bounded away from capacity limits. Precoding is an elegant way to improve the distance spectrum of the transmitter such that the lower bound on the BER decoding performance, can be beaten.

Using precoding, $x_{n}$ is affected by $\mathbf{c}_{n}$ and all previous tuples $\mathbf{c}_{i}, i=0,1, \ldots, n-1$. Vice versa, $P\left(c_{n} \mid \mathbf{y}\right)$ must be computed taking into account the entire sequence $\mathbf{c}$, i.e.,

$$
\begin{array}{r}
P\left(c_{n}=c \mid \mathbf{y}\right)=\sum_{\substack{\mathbf{m} \in \mathbb{F}_{2}^{N}: m_{n}=c \\
\mathbf{m}=\left(m_{0}, m_{1} \ldots, m_{N-1}\right) .}} P(\mathbf{c}=\mathbf{m} \mid \mathbf{y}),
\end{array}
$$

Given a state-space model of the precoder, $P\left(c_{n} \mid \mathbf{y}\right)$ can be obtained using the BCJR algorithm on the combined trellis of the precoder state-space model and the ISI channel state-space model.
For the following precoder state-space model, where the memory $M_{p}$ is restricted to be $q M_{h}$ and the parameter matrices $\mathbf{A}$ and $\mathbf{B}$ are given by

$$
\begin{aligned}
\mathbf{A} & =\left[\begin{array}{ccccc}
\mathbf{A}_{1} & \mathbf{A}_{2} & \mathbf{A}_{3} & \ldots & \mathbf{A}_{M_{h}} \\
\mathbf{I}_{q} & 0 & 0 & \ldots & 0 \\
0 & \mathbf{I}_{q} & 0 & \ldots & 0 \\
\vdots & \vdots & \vdots & \ddots & \vdots \\
0 & 0 & 0 & \ldots & 0
\end{array}\right] \text { and } \\
\mathbf{B} & =\left[\begin{array}{c}
\mathbf{I}_{q} \\
0 \\
\vdots \\
0
\end{array}\right],
\end{aligned}
$$

where the terms $\mathbf{A}_{i}$ are arbitrary $q \times q$ matrices with binary numbers as elements, the state-space models of the channel and precoder can be combined to (48), at the bottom of the page, where $\operatorname{map}\left(\theta_{n}\right)$ is the element-wise mapping from the length- $q M_{h}$ state vector $\theta_{n}$ to $M_{h}$ symbols from $\mathcal{S}$. The corresponding trellis has $2^{q M_{h}}$ states as in the case of no precoding. This holds as well for any precoder with memory $M_{p}$ less than $q M_{h}$, whose parameters $\mathbf{A}$ and $\mathbf{B}$ can be brought into the form (47). Although the constraints in (47) limit the number of possible precoders considerably, the remaining choice turn out to be powerful enough to improve the BER of turbo equalization tremendously.

The extrinsic LLRs $\lambda_{\text {ext }}\left(c_{n}\right)$ are computed as in Section III-B using the combined precoder-channel trellis shown in the second equation at the bottom of the page, with $\mathbf{m}=\left(m_{0}, m_{1}, \ldots, m_{q-1}\right)$, where $\mathbf{f}_{n}$ and $\mathbf{b}_{n}$ are part of the BCJR algorithm and $\mathbf{U}(\mathbf{m})$ returns 1 for all trellis branches with input label $\mathbf{m}$ and 0 otherwise. For example, to compute $\lambda_{\text {ext }}\left(c_{3 n}\right)$ for an 8-PSK alphabet the summation in the numerator might be over the 3-tuples (000), (001), (010), (011) and the summation in the denominator over the 3-tuples (100), (101), (110),(111). The extrinsic transition matrices $\boldsymbol{\Gamma}_{\text {ext }, n}$ are defined as in Section III-B.

$$
\begin{aligned}
& \text { system input : } \quad q \text {-tuples } \mathbf{c}_{n} \text { for } n=0,1, \ldots, L-1 \text {, } \\
& \text { system function : } \theta_{n+1}=\left[\begin{array}{ccccc}
\mathbf{A}_{1} & \mathbf{A}_{2} & \mathbf{A}_{3} & \ldots & \mathbf{A}_{M_{h}} \\
\mathbf{I}_{q} & 0 & 0 & \ldots & 0 \\
0 & \mathbf{I}_{q} & 0 & \ldots & 0 \\
\vdots & \vdots & \vdots & \ddots & \vdots \\
0 & 0 & 0 & \ldots & 0
\end{array}\right] \theta_{n}+\left[\begin{array}{c}
\mathbf{I}_{q} \\
0 \\
\vdots \\
0
\end{array}\right] \mathbf{c}_{n}^{\mathrm{T}} \text {, } \\
& \text { output function: } v_{n}=\left(h_{1} h_{2} \ldots h_{M_{h}}\right) \operatorname{map}\left(\theta_{n}\right)+h_{0} \operatorname{map}\left(x_{n}\right) \text {, } \\
& \text { observation: } \quad y_{n}=v_{n}+w_{n} \text {, }
\end{aligned}
$$

$$
\lambda_{\text {ext }}\left(c_{q n+k}\right)=\ln \frac{\sum_{\mathbf{m} \in \mathbb{F}_{2}^{q}: m_{k}=0} \mathbf{b}_{n+1}^{\mathrm{T}}\left(\mathbf{U}(\mathbf{m}) \odot \boldsymbol{\Gamma}_{\text {ext }, n}\right) \mathbf{f}_{n} \prod_{j=0: j \neq k}^{q-1} \exp \left(-m_{j} \cdot \lambda_{\text {ext }}^{\prime}\left(c_{q n+j}\right)\right)}{\sum_{\mathbf{m} \in \mathbb{F}_{2}^{q}: m_{k}=1} \mathbf{b}_{n+1}^{\mathrm{T}}\left(\mathbf{U}(\mathbf{m}) \odot \boldsymbol{\Gamma}_{\text {ext }, n}\right) \mathbf{f}_{n} \prod_{j=0: j \neq k}^{q-1} \exp \left(-m_{j} \cdot \lambda_{\text {ext }}^{\prime}\left(c_{q n+j}\right)\right)},
$$




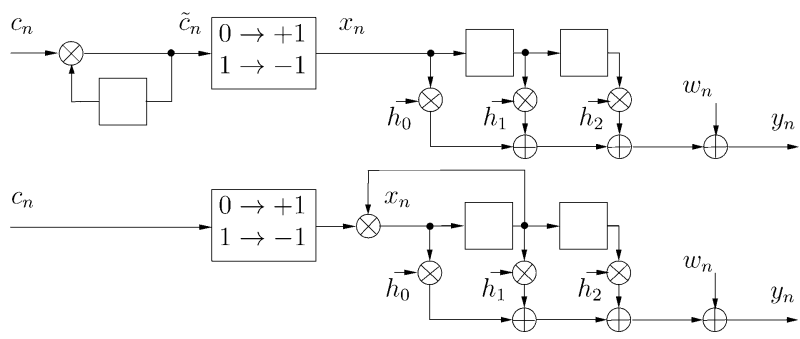

Fig. 14. Two equivalent state machines describing the precoder $\tilde{c}_{n}=c_{n}+$ $\tilde{c}_{n-1}$ followed by a memory-2 ISI channel.

Consider the system configuration in Section II-A, which uses BPSK modulation and a length-3 channel given by the coefficients $h_{0}=0.407, h_{1}=0.815$, and $h_{2}=0.407$. In addition, the memory-1 precoder

$$
\theta_{n+1}=\theta_{n}+c_{n}, \quad \tilde{c}_{n}=\theta_{n}+c_{n}, \quad n=0,1, \ldots, N-1
$$

is applied, i.e., $M_{p}=1, \mathbf{A}=\mathbf{B}=\mathbf{C}=1$, and $\mathbf{D}=1$. The initial state $\theta_{0}$ is 0 . This particular precoding rule can be rewritten to $\tilde{c}_{n}=c_{n}+\tilde{c}_{n-1}$ with $\tilde{c}_{-1}=0$. The parameters $\mathbf{A}$ and $\mathbf{B}$ satisfy the constraint (47), i.e., the combined precoderchannel trellis has only $2^{M_{h}}=4$ states and is derived from the state-space model:

$$
\begin{aligned}
\theta_{n+1} & =\left[\begin{array}{ll}
1 & 0 \\
1 & 0
\end{array}\right] \theta_{n}+\left[\begin{array}{l}
1 \\
0
\end{array}\right] c_{n}, \\
v_{n} & =\left(h_{1} h_{2}\right) \operatorname{map}\left(\theta_{n}\right)+h_{0} \operatorname{map}\left(x_{n}\right), \\
& y_{n}=v_{n}+w_{n} .
\end{aligned}
$$

Fig. 13 depicts the corresponding trellis with the branch labeling $c_{n} / v_{n}$ and Fig. 14 depicts the state machines of the precoder and the ISI channel. The top plot shows both machines separately, such that the combined precoder-channel trellis has $2^{3}=8$ states due to the three memory elements in the system. The bottom plot shows an identical state machine corresponding to (49), which requires only two memory elements. This state reduction is possible, because the precoder satisfies (47).

The BER performance of turbo equalization using the precoder $\tilde{c}_{n}=c_{n}+\tilde{c}_{n-1}$ and symbol-based MAP detection as equalization algorithm is exhibited in Fig. 15 [80] for the same block length $K=510$ as in Fig. 6, which depicts the performance of turbo equalization using symbol-based MAP detection without precoding. Whereas the BER performance of separate equalization and decoding is below that of the system using no precoding shown in Fig. 6, after one iteration the performance bound of the systems using no precoding is beaten.

Another difference to systems without precoding is the strong correlation between the block length $K$ and the number of iterations where performance gains are achieved. This effect has two explanations: The spectrum-enhancing property of precoding is in fact only a necessary condition to achieve improvements in the distance spectrum. The distance enhancement is due to the interleaver, which permutes the ones of the low weight code words $\mathbf{b}$ of the FEC code far away, such that large weights are accumulated in $\tilde{\mathbf{c}}=\mathbf{c M}$ after precoding. An increasing code length $N$ improves this effect, since the interleaver can permute larger groups of consecutive bits farther apart. The bit permutation exerted by the interleaver also helps to fulfill the independence assumption made on the input LLRs to the equalizer and decoder. A system without precoding takes only advantage of the "information decorrelation" purpose of interleaving, which explains why the BER performance over iteration ceases to improve. A system with a carefully designed precoder takes a two-fold advantage of the increasing block length $K$, since both the distance spectrum improves and the interleaver can better decorrelate the input LLRs.

The BER performance of turbo equalization using the precoder $\tilde{c}_{n}=c_{n}+\tilde{c}_{n-1}$ and symbol-based MAP detection as equalization algorithm is exhibited in Fig. 15 for the block length $K=25018$. Compared to Fig. 15, nearly error-free performance is achieved at $2.6 \mathrm{~dB}$ SNR $P / \sigma^{2}$ after 20 iterations. This is only $1.2 \mathrm{~dB}$ away from the capacity limit. The code optimization algorithms in [97], [98] narrow this gap even further.

It is natural to replace the symbol-based MAP detector by an MMSE estimator in case the combined precoder-channel trellis has too many states. Using precoding, the extrinsic LLRs $\lambda_{\text {ext }}\left(c_{n}\right)$ cannot be obtained from $s\left(x_{n}\right)$ using soft demapping as in Sections II-E and III-C, since a particular code bit tuple $\mathbf{c}_{n}$ depends on all symbols $x_{n}$. We note that $\lambda_{\text {ext }}\left(c_{n}\right)$ must be computed by marginalizing $c_{n}$ in the joint probabilities $P(\mathbf{c} \mid \hat{\mathbf{x}})$ with $\hat{\mathbf{x}}=\left(\hat{x}_{0} \hat{x}_{1} \ldots \hat{x}_{L-1}\right)^{\mathrm{T}}$. This can be done efficiently with the BCJR algorithm using the precoder trellis, where the transition matrices $\boldsymbol{\Gamma}_{n}$ and $\boldsymbol{\Gamma}_{\text {ext, } n}$ are constructed with the corresponding probabilities $s\left(x_{n}\right)$.

However, the BCJR algorithm is derived based on the assumption that the observations $\hat{x}_{n}$ of the output $\tilde{\mathbf{c}}_{n}$ or $x_{n}=$ $\operatorname{map}\left(\tilde{\mathbf{c}}_{n}\right)$, respectively, of the precoder state-space model are conditionally independent given $x_{n}$, e.g., $p\left(\hat{x}_{n}, \hat{x}_{k} \mid x_{n}, x_{k}\right)=$ $p\left(\hat{x}_{n} \mid x_{n}\right) p\left(\hat{x}_{k} \mid x_{k}\right)$. This is not the case with the estimates $\hat{x}_{n}$ produced by the MMSE estimator.

A remedy to this problem is to place an interleaver between the precoder output $\tilde{\mathbf{c}}$ and the mapper to $\mathbf{x}$. The resulting cascade FEC code-interleaver-precoder-interleaver-mapping is a three-fold concatenation, for which the turbo equalization algorithm in Fig. 5 must take the form of other iterative decoders for three-fold concatenated systems, as in [99]-[103].

\section{CONCLUSION}

This paper provides an overview of turbo equalization, including some of its basic concepts, applications, and tools for analysis. Beginning with the simple observation of [2] that an intersymbol-interference channel could be viewed as a rate-1 convolutional code over the reals, turbo equalization was simply an extension of the turbo decoding algorithm applied to a coded data transmission over a frequency selective channel. By considering the channel itself as an inner-code of a serially concatenated turbo code, the problems of equalization and decoding became inextricably linked.

Two critical components are the communication of extrinsic information between the equalizer and the decoder and the use of interleaving to enable the independence assumptions made at the equalizer and decoder to facilitate simple operation. As such, 


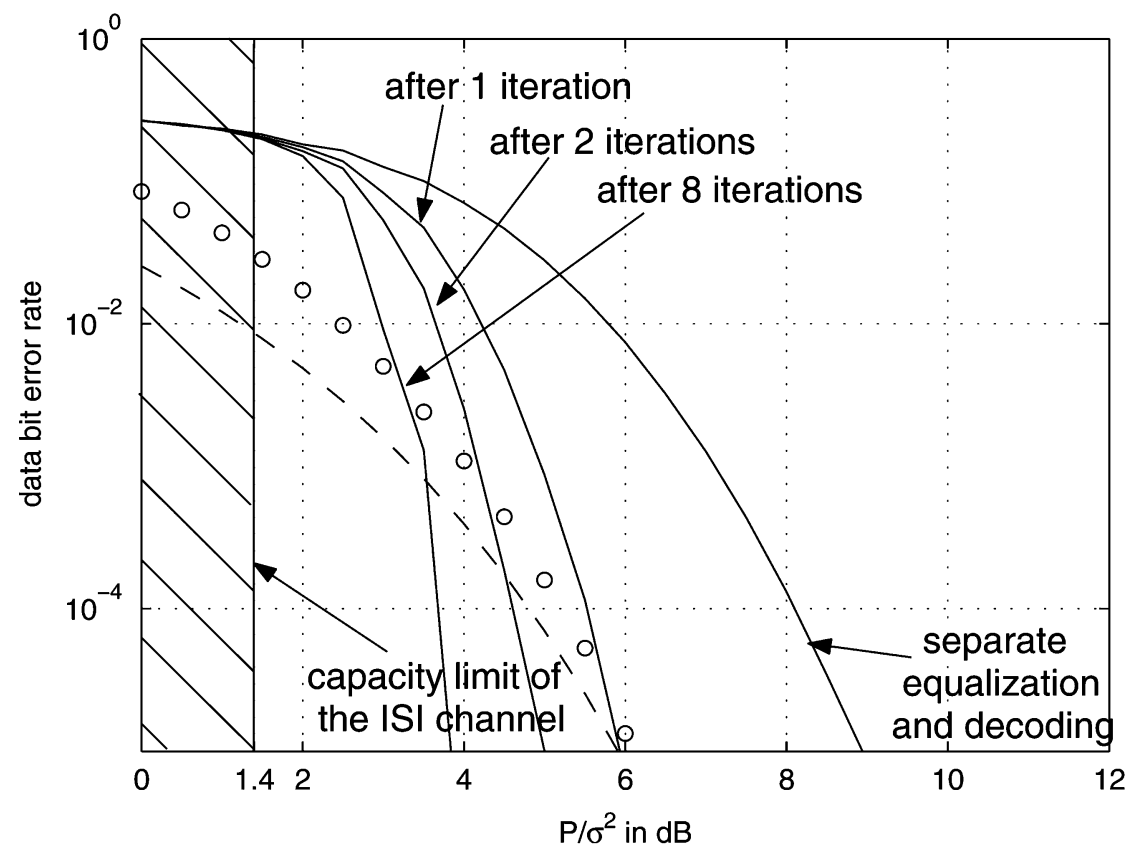

Fig. 15. Performance of turbo equalization for the communication system described in Section II-A using the precoder $\tilde{c}_{n}=c_{n}+\tilde{c}_{n-1}$ and symbol-based MAP detection. The BER performance (solid lines) is plotted for separate equalization and decoding as well as after 1, 2, or 8 iterations. The dotted line corresponds to the BER performance of the FEC decoder when no ISI is introduced in the channel. The dashed line is a lower bound on the BER performance of any decoder when no precoding is used in the transmitter. The considered blocklength is $K=510(N=L=1024)$. An S-random interleaver with S $=16$ is applied.

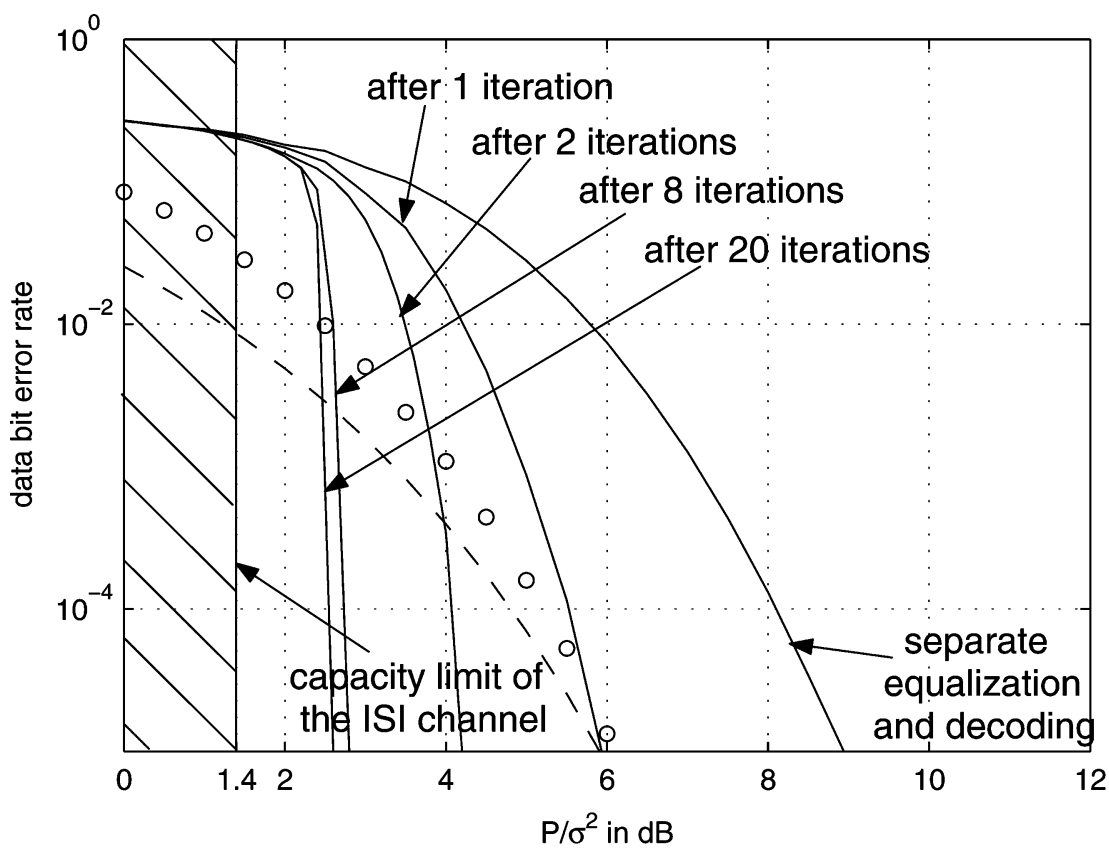

Fig. 16. Performance of turbo equalization for the communication system described in Section II-A using the precoder $\tilde{c}_{n}=c_{n}+\tilde{c}_{n-1}$ and symbol-based MAP detection. The BER performance (solid lines) is plotted for separate equalization and decoding as well as after 1, 2, 8, or 20 iterations. The dotted line corresponds to the BER performance of the FEC decoder when no ISI is introduced in the channel. The dashed line is a lower bound on the BER performance of any decoder when no precoding is used in the transmitter. The considered blocklength is $K=25018(N=L=50040)$. An S-random interleaver with S $=40$ is applied.

turbo equalization requires that both the equalizer and the decoder be capable of processing and outputing soft information, i.e., they need to have SISO capability. While symbol-based MAP detection and MAP decoding are natural SISO equalization algorithms, Koetter's work, and that of others, showed that MMSE estimation (and a host of other serially concatenated tasks) can be used to construct a SISO algorithm as well. An important observation is that the turbo equalization algorithm using either symbol-based MAP detection or linear MMSE estimation often achieves the BER performance of ML decoding for the complete communication link after convergence, i.e., turbo equalization has performance that approaches that of the exact joint equalization and decoding. However, a lower bound on the BER governed by the minimum distance $d_{\min \text {,fec }}$ of the 
FEC code is bounded away from the capacity limit, when the ISI channel has finite memory. Precoding, however, provides a simple means for overcoming this performance limitation and in certain cases can be designed without an increase in receiver complexity.

We see that the turbo equalization approach is tightly connected to recent work in a variety of areas, including turbo codes, LDPC codes, and iterative detection and decoding algorithms. Just as turbo equalization grew out of the original turbo decoding algorithm, there are numerous extensions of the basic turbo equalization approach in which a wide variety of signal processing tasks have been incorporated into the joint estimation process. There are a host of additional means in which to explore the turbo equalization approach, and we refer the interested reader to the references cited and the references contained therein. A few of the important open problems in this area include: a detailed theoretical analysis of the convergence and asymptotic performance of turbo equalization; operation in the presence of unknown and time-varying channels and the relative merits of frequency-domain and time-domain formulations in this context; complexity-reduction approaches to turbo equalization for MIMO-ISI channels and the development of other non-trellis-based detection methods that provide performance between that of MMSE and MAP performance. We hope that this overview of the general concepts and salient features enables further exploration into this topic that we find both challenging and fascinating.

\section{REFERENCES}

[1] C. Berrou and A. Glavieux, "Near optimum error correcting coding and decoding: Turbo codes," IEEE Trans. Commun., vol. 44, no. 10, pp. 1261-1271, Oct. 1996.

[2] C. Douillard, M. Jezequel, and C. Berrou, "Iterative correction of intersymbol interference: Turbo equalization," Eur. Trans. Telecommun., vol. 6, no. 5, pp. 507-511, Sep.-Oct. 1995.

[3] J. Proakis, Digital Communications, 3rd ed. New York: McGrawHill, 1995.

[4] S. Haykin, Communication Systems, 3rd ed. Toronto, ON, Canada: Wiley Canada, 1994.

[5] S. Lin and J. Costello, Error Control Coding. Englewood Cliffs, NJ: Prentice-Hall, 1983.

[6] G. Forney, "Maximum-likelihood estimation of digital sequences in the presence of intersymbol interference," IEEE Trans. Inf. Theory, vol. IT-18, no. 3, pp. 363-378, May 1972.

[7] L. Bahl, J. Cocke, F. Jelinek, and J. Raviv, "Optimal decoding of linear codes for minimizing symbol error rate," IEEE Trans. Inf. Theory, vol. IT-20, pp. 284-287, Mar. 1974.

[8] Y. Li, B. Vucetic, and Y. Sato, "Optimum soft-output detection for channels with intersymbol interference," IEEE Trans. Inf. Theory, vol. 41, no. 3, pp. 704-713, May 1995.

[9] W. Koch and A. Baier, "Optimum and sub-optimum detection of coded data disturbed by time-varying intersymbol interference," in Proc. IEEE Global Telecommun. Conf., Dec. 1990, pp. 1679-1684.

[10] C. Berrou, A. Glavieux, and P. Thitimajshima, "Near Shannon limit error-correcting coding and decoding: Turbo codes," in Proc. IEEE Int. Conf. Commun. (ICC), May 1993.

[11] C. Heegard and S. Wicker, Turbo Coding. Boston, MA: Kluwer, 1999.

[12] J. Hagenauer, "The turbo principle: Tutorial introduction and state of the art," in Proc. Int. Symp. Turbo Codes Related Topics, Brest, France, Sep. 1997, pp. 1-11.

[13] F. R. Kschischang, B. Frey, and H.-A. Loeliger, "Factor graphs and the sum-product algorithm," IEEE Trans. Inf. Theory, vol. 47, pp. 498-519, Feb. 2001.

[14] H. Niu, M. Shen, J. Ritsey, and H. Liu, "Iterative channel estimation and LDPC decoding over flat-fading channels: A factor graph approach," in Proc. Conf. Inf. Sci. Syst. (CISS), Mar. 2003.
[15] A. Worthen and W. Stark, "Unified design of iterative receivers using factor graphs," IEEE Trans. Inf. Theory, vol. 47, no. 2, pp. 843-849, Feb. 2001.

[16] R. Drost and A. Singer, "Factor-graph algorithms for equalization," IEEE Trans. Signal Process., vol. 55, no. 5, pp. 2052-2065, May 2007.

[17] S. Benedetto, D. Divsalar, G. Mondorsi, and F. Pollara, "Serial concatenated trellis coded modulation with iterative decoding: Design and performance," presented at the IEEE Global Telecommun. Conf., Phoenix, AZ, Nov. 1997.

[18] S. Benedetto and G. Mondorsi, "Unveiling Turbo codes: Some results on parallel concatenated coding schemes," IEEE Trans. Inf. Theory, vol. 42, no. 2, pp. 409-428, Mar. 1996.

[19] X. Wang and H. Poor, "Iterative (turbo) soft interference cancellation and decoding for coded CDMA," in IEEE Int. Conf. Commun. (ICC), 1999, vol. 47, no. 7, pp. 1046-1061.

[20] A. Singer, J. Nelson, and S. Kozat, "Signal processing for underwater acoustic communications," IEEE Commun. Mag., vol. 47, no. 1, pp. 90-96, Jan. 2009.

[21] J. Sifferlen, H. Song, W. Hodgkiss, W. Kuperman, and M. Stevenson, "An iterative equalization and decoding approach for underwater acoustic communication," IEEE J. Ocean. Eng., pp. 182-197, 2008.

[22] J. Choi, R. Drost, A. Singer, and J. Preisig, "Iterative multi-channel equalization and decoding for high frequency underwater acoustic communications," in Proc. 2008 IEEE Sensor Array Multichannel Signal Process. Workshop, Jul. 2008, pp. 127-130.

[23] R. Otnes and T. H. Eggen, "Underwater acoustic communications: Long-term test of turbo equalization in shallow water," IEEE J. Ocean. Eng., vol. 33, no. 3, pp. 321-334, Jul. 2008.

[24] F. Xu, M.-A. Khalighi, and S. Bourennane, "Coded PPM and multipulse PPM and iterative detection for free-space optical links," IEEE/OSA J. Opt. Commun. Netw., vol. 1, no. 5, pp. 404-415, Oct. 2009.

[25] H. Haunstein, T. Schorr, A. Zottmann, W. Sauer-Greff, and R. Urbansky, "Performance comparison of MLSE and iterative equalization in FEC systems for pmd channels with respect to implementation complexity," Journal of Lightwave Technol., vol. 24, no. 11, pp. 4047-4054, Nov. 2006.

[26] H. Haunstein, W. Sauer-Greff, A. Dittrich, K. Sticht, and R. Urbansky, "Principles for electronic equalization of polarization-mode dispersion," J. Lightw. Technol., vol. 22, no. 4, pp. 1169-1182, Apr. 2004.

[27] G. Bauch and V. Franz, "A comparison of soft-in/soft-out algorithms for 'turbo detection'," in Proc. Int. Conf. Telecommun. (ICT), Jun. 1998, pp. 259-263.

[28] A. Anastasopoulos and K. Chugg, "Iterative equalization and decoding for TCM for frequency-selective fading channels," in Proc. Allerton Conf. Commun., Control, Comput., Monticello, IL, Nov. 1997, vol. 1, pp. $177-181$.

[29] M. Tüchler, R. Koetter, and A. Singer, "Turbo equalization: Principles and new results," IEEE Trans. Commun., vol. 50, no. 5, pp. 754-767, May 2002.

[30] M. Tüchler, A. Singer, and R. Koetter, "Minimum mean squared error equalization using priors," IEEE Trans. Signal Process., vol. 50, no. 3, pp. 673-683, Mar. 2002.

[31] J. Hagenauer and P. Hoeher, "A Viterbi algorithm with soft-decision outputs and its applications," in Proc. IEEE Global Telecommun. Conf., 1989 , pp. $1680-1686$.

[32] S. Lee, N. Shanbhag, and A. Singer, "A 285-MHz map decoder in $0.18 \mu \mathrm{m}$ CMOS," IEEE J. Solid-State Circuits, vol. 40, no. 8, pp. 1718-1725, Aug. 2005.

[33] S.-J. Lee, N. Shanbhag, and A. Singer, "Area-efficient high-throughput map decoder architectures," IEEE Trans. Very Large Scale Integr. (VLSI) Syst., vol. 13, no. 8, pp. 921-933, Aug. 2005.

[34] R. Ratnayake, A. Kavcic, and G.-Y. Wei, "A high-throughput maximum a posteriori probability detector," IEEE J. Solid-State Circuits, vol. 43, no. 8, pp. 1846-1858, Aug. 2008.

[35] J. Erfanian, S. Pasupathy, and P. G. Gulak, "Reduced complexity symbol detectors with parallel structure for ISI channels," IEEE Trans. Commun., vol. 42, pp. 1661-1671, 1994.

[36] E. Boutillon, W. J. Gross, and P. G. Gulak, "Vlsi architectures for the MAP algorithm," IEEE Trans. Commun., vol. 51, pp. 175-185, Feb. 2003.

[37] A. Worm, H. Lamm, and N. Wehn, "Vlsi architectures for high-speed MAP decoders," in Proc. 14th Int. Conf. VLSI Design, 2001, pp. 446-453.

[38] H. Dawid, G. Gehnen, and H. Meyr, "MAP channel decoding: Algorithm and VLSI architecture," in 1993 Workshop VLSI Signal Process., Veldhoven, The Netherlands, Oct. 1993, pp. 141-149. 
[39] A. Glavieux, C. Laot, and J. Labat, "Turbo equalization over a frequency selective channel," in Proc. Int. Symp. Turbo Codes Related Topics, Brest, France, Sep. 1997, pp. 96-102.

[40] D. Raphaeli and A. Saguy, "Linear equalizers for turbo equalization: A new optimization criterion for determining the equalizer taps," in Proc. Int. Symp. Turbo Codes Related Topics, Brest, France, Sep. 2000, pp. 371-374.

[41] Z. Wu and J. Cioffi, "Turbo decision aided equalization for magnetic recording channels," in Proc. IEEE Global Telecommun. Conf., Dec. 1999, pp. 733-738.

[42] R. Lopes and J. Barry, "The soft-feedback equalizer for turbo equalization of highly dispersive channels," IEEE Trans. Commun., vol. 54, no. 5, pp. 783-788, May 2006.

[43] L. Liu and L. Ping, "An extending window MMSE turbo equalization algorithm," IEEE Signal Process. Lett., vol. 11, no. 11, pp. 891-894, Nov. 2004.

[44] A. Berthet, R. Visoz, and P. Tortelier, "Sub-optimal turbo-detection for coded 8-PSK signals over ISI channels with application to EDGE advanced mobile system," in Proc. IEEE Veh. Technol. Conf. (VTC), Sep. 2000.

[45] D. Fertonani, A. Barbieri, and G. Colavolpe, "Reduced-complexity BCJR algorithm for turbo equalization," IEEE Trans. Commun., vol. 55, no. 12, pp. 2279-2287, Dec. 2007.

[46] F. Kashif, H. Wymeersch, and M. Win, "Monte Carlo equalization for nonlinear dispersive satellite channels," IEEE J. Sel. Areas Commun., vol. 26, no. 2, pp. 245-255, Feb. 2008.

[47] R. Peng, R.-R. Chen, and B. Farhang-Beroujeny, "Markov chain Monte Carlo detectors for channels with intersymbol interference," IEEE Trans. Signal Process., vol. 58, no. 4, pp. 2206-2217, Apr. 2010.

[48] Z. Qin, K. Cai, and X. Zou, "A reduced-complexity iterative receiver based on simulated annealing for coded partial-response channels," IEEE Trans. Magn., vol. 43, no. 6, pp. 2265-2267, Jun. 2007.

[49] Z. Qin and K. C. Teh, "Reduced-complexity turbo equalization for coded intersymbol interference channels based on local search algorithms," IEEE Trans. Veh. Technol., vol. 57, no. 1, pp. 630-635, Jan. 2008.

[50] P. Supnithi, R. Lopes, and S. McLaughlin, "Reduced-complexity turbo equalization for high-density magnetic recording systems," IEEE Trans. Magn., vol. 39, no. 5, pp. 2585-2587, Sep. 2003.

[51] N. Singla, J. O'Sullivan, R. Indeck, and Y. Wu, "Iterative decoding and equalization for 2-D recording channels," IEEE Trans. Magn., vol. 38, pp. 2328-2330, Sep. 2002.

[52] Y. Wu, J. O'Sullivan, N. Singla, and R. Indeck, "Iterative detection and decoding for separable two-dimensional intersymbol interference," IEEE Trans. Magn., vol. 39, no. 4, pp. 2115-2120, Jul. 2003.

[53] N. Singla, J. O'Sullivan, C. Miller, and R. Indeck, "Decoding for magnetic recording media with overlapping tracks," IEEE Trans. Magn., vol. 41, no. 10, pp. 2968-2970, 2005.

[54] H. Li and H. Poor, "Reduced complexity joint iterative equalization and multiuser detection in dispersive DS-CDMA channels," IEEE Trans. Wireless Commun., vol. 4, no. 3, pp. 1234-1243, May 2005.

[55] T. Mayer and C. Kuhn, "Distributed turbo equalization for intercell and intersymbol interference cancellation," in IEEE Global Telecommun. Conf., 2006, 2006, pp. 1-5.

[56] D. Lin and T. J. Lim, "A variational inference framework for soft-in soft-out detection in multiple-access channels," IEEE Trans. Inf. Theory, vol. 55, no. 5, pp. 2345-2364, May 2009.

[57] P. Xiao, J. Wu, M. Sellathurai, T. Ratnarajah, and E. Strom, "Iterative multiuser detection and decoding for DS-CDMA system with spacetime linear dispersion," IEEE Trans. Veh. Technol., vol. 58, no. 5, pp. 2343-2353, Jun. 2009.

[58] T. Abe, S. Tomisato, and T. Matsumoto, "A MIMO turbo equalizer for frequency-selective channels with unknown interference," IEEE Trans. Veh. Technol., vol. 52, no. 3, pp. 476-482, May 2003.

[59] M. Koca and B. Levy, "Broadband beamforming for joint interference cancellation and turbo equalization," IEEE Trans. Wireless Commun., vol. 4, no. 5, pp. 2244-2255, Sep. 2005.

[60] T. Ait-Idir, S. Saoudi, and N. Naja, "Space-time turbo equalization with successive interference cancellation for frequency-selective MIMO channels," IEEE Trans. Veh. Technol., vol. 57, no. 5, pp. 2766-2778, Sep. 2008.

[61] S.-S. Sadough, M.-A. Khalighi, and P. Duhamel, "Improved iterative MIMO signal detection accounting for channel-estimation errors," IEEE Trans. Veh. Technol., vol. 58, no. 7, pp. 3154-3167, Sep. 2009.

[62] M. Sellathurai and S. Haykin, "T-blast for wireless communications: First experimental results," IEEE Trans. Veh. Technol., vol. 52, no. 3, pp. 530-535, May 2003.
[63] M. Koca and B. Levy, "Turbo space-time equalization of TCM for broadband wireless channels," IEEE Trans. Wireless Commun., vol. 3 , no. 1, pp. 50-59, Jan. 2004.

[64] K. Kansanen, C. Schneider, T. Matsumoto, and R. Thoma, "Multilevelcoded QAM with MIMO turbo-equalization in broadband single-carrier signaling," IEEE Trans. Veh. Technol., vol. 54, no. 3, pp. 954-966, May 2005.

[65] S. Ahmed, T. Ratnarajah, M. Sellathurai, and C. Cowan, "Reduced-complexity iterative equalization for severe time-dispersive MIMO channels," IEEE Trans. Veh. Technol., vol. 57, no. 1, pp. 594-600, Jan. 2008.

[66] R. Visoz, A. O. Berthet, and S. Chtourou, "Frequency-domain block turbo-equalization for single-carrier transmission over MIMO broadband wireless channel," IEEE Trans. Commun., vol. 54, pp. 2144-2149, Dec. 2006.

[67] F. Pancaldi and G. Vitetta, "Block channel equalization in the frequency domain,” IEEE Trans. Commun., vol. 53, no. 3, pp. 463-471, Mar. 2005.

[68] H. Liu and P. Schniter, "Iterative frequency-domain channel estimation and equalization for single-carrier transmissions without cyclic-prefix," IEEE Trans. Wireless Commun., vol. 7, no. 10, pp. 3686-3691, Oct. 2008.

[69] C. Laot, R. L. Bidan, and D. D. Leroux, "Low-complexity MMSE turbo equalization: A possible solution for EDGE," IEEE Trans. Wireless Commun., vol. 4, no. 3, pp. 965-974, May 2005.

[70] D. N. Liu and M. P. Fitz, "Low complexity affine MMSE detector for iterative detection-decoding MIMO-OFDM systems," IEEE Trans. Commun., vol. 56, no. 1, pp. 150-158, 2008.

[71] S. Ahmed, T. Ratnarajah, M. Sellathurai, and C. Cowan, "Iterative receivers for MIMO-OFDM and their convergence behavior," IEEE Trans. Veh. Technol., vol. 58, no. 1, pp. 461-468, Jan. 2009.

[72] Y. Sun, V. Tripathi, and M. L. Honig, "Adaptive turbo reduced-rank equalization for MIMO channels," IEEE Trans. Wireless Commun, vol 4, pp. 2789-2800, 2005.

[73] R. Otnes and M. Tuchler, "Iterative channel estimation for turbo equalization of time-varying frequency-selective channels," IEEE Trans. Wireless Commun., vol. 3, no. 6, pp. 1918-1923, Nov. 2004.

[74] S. Song, A. Singer, and K.-M. Sung, "Soft input channel estimation for turbo equalization," IEEE Trans. Signal Process., vol. 52, no. 10, pp. 2885-2894, Oct. 2004.

[75] X. Li and T. F. Wong, "Turbo equalization with nonlinear Kalman filtering for time-varying frequency-selective fading channels," IEEE Trans. Wireless Commun., vol. 6, no. 2, pp. 691-700, Feb. 2007.

[76] K. Fang, L. Rugini, and G. Leus, "Low-complexity block turbo equalization for OFDM systems in time-varying channels," IEEE Trans. Signal Process., vol. 56, no. 11, pp. 5555-5566, 2008.

[77] Q. Yu and S. Lambotharan, "Iterative (turbo) estimation and detection techniques for frequency-selective channels with multiple frequency offsets," IEEE Signal Process. Lett., vol. 14, no. 4, pp. 236-239, Apr. 2007.

[78] S. Das and P. Schniter, "Max-SINR ISI/ICI-shaping multicarrier communication over the doubly dispersive channel," IEEE Trans. Signal Process., vol. 55, no. 12, pp. 5782-5795, Dec. 2007.

[79] J. Hagenauer, E. Offer, and L. Papke, "Iterative decoding of binary block and convolutional codes," IEEE Trans. Inf. Theory, vol. 42, no. 3, pp. 429-445, Mar. 1996.

[80] R. Koetter, A. Singer, and M. Tüchler, "Turbo equalization," IEEE Signal Process. Mag., vol. 21, no. 1, pp. 67-80, Jan. 2004.

[81] P. Robertson, P. Hoeher, and E. Villebrun, "Optimal and sub-optima maximum a posteriori algorithms suitable for turbo decoding," Eur. Trans. Telecommun., vol. 8, pp. 119-125, 1997.

[82] A. J. Viterbi, "An intuitive justification and a simplified implementation of the MAP decoder for convolutional codes," IEEE J. Sel. Areas Commun., vol. 16, no. 2, pp. 260-264, Feb. 1998.

[83] J. Proakis and M. Salehi, Communication Systems Engineering. Upper Saddle River, NJ: Prentice Hall, 1994.

[84] H. Poor, An Introduction to Signal Detection and Estimation, 2nd ed. New York: Springer-Verlag, 1994.

[85] M. Tüchler, R. Koetter, and A. Singer, "Graphical models for coded data transmission over inter-symbol interference channels," presented at the IEEE/ITG Conf. Source Channel Coding, Erlangen, Germany, Jan. 2004.

[86] M. Tüchler, R. Koetter, and A. Singer, "Graphical models for coded data transmission over inter-symbol interference channels," Eur. Trans. Telecommun., vol. 15, no. 4, pp. 307-321, Jul.-Aug. 2004.

[87] M. Moher and T. Gulliver, "Cross-entropy and iterative decoding," IEEE Trans. Inf. Theory, vol. 44, no. 7, p. 1998, Nov. 1998. 
[88] G. Golub and C. van Loan, Matrix Computations, 3rd ed. Baltimore, MD: Johns Hopkins Univ. Press, 1996.

[89] C. Caire, G. Taricco, and E. Biglieri, "Bit-interleaved coded modulation,” IEEE Trans. Inf. Theory, vol. 44, no. 3, pp. 927-946, May 1998.

[90] S. ten Brink, J. Speidel, and R. Yan, "Iterative demapping and decoding for multilevel modulation," in Proc. IEEE Global Commun. Conf. (Globecom), Nov. 1998, pp. 579-584.

[91] W. Gerstacker, R. Schober, and A. Lampe, "Receivers with widely linear processing for frequency-selective channels," IEEE Trans. Commun., vol. 51, no. 9, pp. 1512-1523, Sep. 2003.

[92] S. Jiang, L. Ping, H. Sun, and C. S. Leung, "Modified LMMSE turbo equalization," IEEE Commun. Lett., vol. 8, no. 3, pp. 174-176, Mar. 2004.

[93] R. Otnes, "On soft-output demapping in filter-based soft-in/soft-out equalizers," presented at the Int. Symp. Turbo Codes Related Topics, Brest, France, Sep. 2003.

[94] R. V. Nee and R. Prasad, OFDM for Wireless Multimedia Communications. Norwood, MA: Artech House, 2000.

[95] R. Ramirez, The FFT: Fundamentals and Concepts. Upper Saddle River, NJ: Prentice-Hall, 1984.

[96] M. Tüchler and J. Hagenauer, "Turbo equalization using frequency domain equalizers," in Proc. Allerton Conf. Commun., Control, Computing, Monticello, IL, Oct. 2000, pp. 1234-1243.

[97] M. Tüchler, "Design of serially concatenated systems depending on the block length," presented at the Proc. IEEE Int. Conf. Commun. (ICC), Anchorage, AL, May 2003.

[98] M. Tüchler, "Design of serially concatenated systems depending on the block length," IEEE Trans. Commun., vol. 52, no. 2, pp. 209-218, Feb. 2004.

[99] M. Tüchler, "Convergence prediction of iterative decoding of threefold concatenated systems," presented at the IEEE Global Commun. Conf. (Globecom), Taipei, Taiwan, Nov. 2002.

[100] R. Ramamurthy and W. Ryan, "Convolutional double accumulate codes (or double turbo DPSK)," IEEE Commun. Lett., vol. 5, no. 4, pp. 157-159, Apr. 2001.

[101] D. Raphaeli and Y. Zarai, "Combined turbo equalization and turbo decoding," IEEE Commun. Lett., vol. 2, no. 4, pp. 107-109, Apr. 1998.

[102] S. ten Brink, "Code doping for triggering iterative decoding convergence," in Proc. Int. Symp. Inf. Theory (ISIT), Oct. 2001, p. 235.
[103] M. Toegel, W. Pusch, and H. Weinrichter, "Combined serially concatenated codes and turbo-equalization," in Proc. Int. Symp. Turbo Codes Related Topics, Brest, France, Sep. 2000, pp. 375-378.

[104] M. Tüchler, "Turbo equalization," Ph.D. Dissertation, Munich Univ. Technol., Munich, Germany, Dec. 2003.

Michael Tüchler received the B.Sc. degree from Ilmenau University of Technology, Germany, in 1998, the M.Sc. degree from the University of Illinois, Urbana-Champaign, in 2000, the M.B.A. degree from the College des Ingenieurs, Paris, France, in 2004, and the Ph.D. degree from the Munich University of Technology, Germany, in 2003.

From 2005 to 2006, he was a Project Engineer at the Center for Microelectronics Aargau, Zurich, Switzerland, and from 2006 to 2008 he was a Development Engineer at Oerlikon Space AG. He currently serves as a Systems Engineer at Rheinmetall, AG.

Andrew C. Singer (S'92-SM'96-M'96-SM'05-F'10) received the S.B., S.M., and $\mathrm{Ph} . \mathrm{D}$. degrees, all in electrical engineering and computer science, from the Massachusetts Institute of Technology, Cambridge.

From 1996 to 1998, he was a Research Scientist at Sanders, a Lockheed Martin company, Manchester, NH. Since 1998, he has been on the faculty of the Department of Electrical and Computer Engineering, University of Illinois, Urbana-Champaign, where he is currently a Professor in the ECE Department and Director of the Technology Entrepreneur Center for the College of Engineering. His research spans algorithms and architectures for statistical signal processing and communication systems.

Dr. Singer is a Member of the MIT Educational Council, and of Eta Kappa $\mathrm{Nu}$ and Tau Beta Pi. He was a Hughes Aircraft Masters Fellow, and was the recipient of the Harold L. Hazen Memorial Award for excellence in teaching in 1991. In 2000, he received the National Science Foundation CAREER Award, in 2001 he received the Xerox Award for Outstanding Faculty Research, and in 2002 was named a Willett Faculty Scholar. He has received Best Paper Awards from the IEEE Solid State Circuits Society in 2006, and in 2008 from the IEEE Signal Processing Society. 